\title{
Uppsala University Annual Bibliometric Monitoring 2019
}

\author{
Per Ahlgren ${ }^{1}$, Leif Eriksson ${ }^{1}$, Daniel Wadskog ${ }^{2}$, and Erik Åkesson Kågedal ${ }^{2}$ \\ ${ }^{1}$ Planning Division, Uppsala University \\ ${ }^{2}$ Uppsala University Library
}

\section{Introduction}

Nowadays, and in several countries in the world, bibliometric evaluation of research is performed to a quite large extent. In the Swedish context, bibliometric analysts at Karolinska Institutet and KTH Royal Institute of Technology, for instance, perform annual bibliometric analyses of the publication output of these universities.

Uppsala University (UU) Annual Bibliometric Monitoring (ABM) produces statistics on publication volume, publishing level, citation impact, collaboration (in terms of co-publishing) and open access (OA). The aggregation level is primarily department, which means that it is primarily the departments of UU that are the units of analysis in ABM. However, the first subsection of the results section below reports outcomes for UU as a whole.

Values of several bibliometric indicators are obtained for the departments included in ABM. Indicator values are obtained by publication year. However, 3-year moving averages are generally applied.

In "Quality and Renewal 2017" ("Kvalitet och förnyelse 2017") (Malmberg et al., 2017), the peer assessments were complemented with a bibliometric report. One conclusion of the exercise was that an annual bibliometric report was desirable. The ABM 2019 is a response to this conclusion and presents extensive bibliometric statistics with regard to the UU departments.

The remainder of this report is structured as follow. Section 2 treats the data and methods of ABM. The results of ABM are reported in Section 3, whereas the results are briefly discussed in Section 4. Concluding remarks are given in Section 5.

\section{Data and methods}

Two main data sources are used in ABM: the local publication database of UU, DiVA, and the in-house version of Web of Science (WoS) database available at the Centre for Science and Technology Studies (CWTS) at Leiden University, the Netherlands. The latter database is accessed via CWTS Monitor, a tool for bibliometric analysis of WoS publications and provided by CWTS. We used version 2019/2 in ABM 2019. DiVA data, or more precisely DiVA data as it appear in GLIS (Generellt Lednings Informations System), are used for the analysis according to the Norwegian model, whereas CWTS Monitor is used for the analysis of citation impact and collaboration. For OA, DiVA data is used from DiVA directly but combined with data from GLIS, $\mathrm{WoS}^{1}$, and Directory of Open Access Journals (DOAJ).

In the Norwegian model, as well as in the citation analyses in ABM, author fractionalization is used. An author of a publication is assigned $1 / n$ of the publication, where $n$ is the number of authors of the publication. The fraction of the publication assigned to a given UU department is the sum of the author fractions with regard to the authors affiliated to the department. Note, though, that if an author is affiliated to $m(m \geq 2)$ UU departments in a publication, the author contributes with $1 /(n \times m)$ author fraction to all $m$ departments (see Appendix 2).

\footnotetext{
${ }^{1}$ Since WoS does not cover all UU publications, the levels of different OA classes might vary between different research fields. We use, however, information in DiVA and DOAJ to classify all UU publications, even those not covered by WoS.
} 
In the citation analyses and in the collaboration analyses of ABM, only the WoS publication types "Article" and "Review" are taken into account. Further, in these two types of analyses, only core publications are used. Core publications are publications in international scientific journals in fields that are suitable for citation analysis. ${ }^{2}$ In the citation analyses, author self-citations are excluded. A citation is counted as an author self-citation if the citing and the cited publication have at least one common author name.

For open access, we use whole counts at the department level: each affiliated department is assigned the publication. OA is measured by defining the open access status for each publication and counting the number of publications affiliated to each department.

We use the following condition for department inclusion in the Norwegian model, the collaboration and the OA analyses:

(a) The researchers of the department have not their main affiliation to another unit.

Information on (a) was obtained from the offices for the three scientific domains of UU. These three domains are Humanities and Social Sciences (HumSam), Medicine and Pharmacy (MedFarm), and Science and Technology (TekNat).

With regard to the citation analyses of $\mathrm{ABM}$, the following two inclusion conditions are applied.

(a) the researchers of the department have not their main affiliation to another unit, and

(b) the department has at least 40 publications (of the types "Article" and "Review") in the period 2010-2017.

For each table below that reports citation analysis results, the expression "null" in a cell indicates that condition (a), but not condition (b), is satisfied by the department corresponding to the row of the cell.

\subsection{The Norwegian model}

The Norwegian model considers publishing volume and publishing level. For level, the model does not use citations. Instead, the model takes into account the extent to which publications from a unit of analysis are published in publishing channels with large prestige. The channels that are considered in the model are journals, publishers and series. A large number of channels have been assessed in Norway by subject experts and assigned to one of three levels: level 0 (non-scientific channel), level 1 (scientific channel), and level 2 (scientific channel with extra-large prestige).

The Norwegian model comprises three publication types: article in journal or series, article in anthology and monograph. Conference papers are taken into account. If the host publication of a conference paper belongs to a series, the paper is classified as article in journal or series, otherwise the paper is classified as article in anthology. The weight of a publication is determined by its type and by the level of its channel. How publications are weighted is reported in Table 1.

Table 1. The weights of the Norwegian model as a function of publication type and publishing level.

\begin{tabular}{lrr}
\hline Publication type & Level 1 & Level 2 \\
\hline Article in journal or series & 1 & 3 \\
Article in anthology & 0,7 & 1 \\
Monograph & 5 & 8 \\
\hline
\end{tabular}

The publishing channels at level 2 in a given research field should publish a certain proportion (20\%) of the publications of the field. By this rule, comparisons across fields are fairer, compared to if a certain number of channels, constant across fields, had been stipulated for level 2 . The reason for this is that the

\footnotetext{
${ }^{2}$ For more on core journals, see the CWTS Leiden Ranking page https://www.leidenranking.com/information/indicators.
} 
access to level 2 channels becomes more equal across fields. Note that the proportion publications at level 2 for a given unit of analysis can be seen as a quality indicator (Schneider, 2009).

Some publications are excluded in analyses according to the Norwegian model, for instance:

- Letter to the editor

- Working reports

- Publications where the author has editorship

- Popular scientific publications

Each unit of analysis is assigned a publication score, a weighted expression for publishing volume and publishing level. The scores are obtained by multiplying author fractions by weights. For example, the publication score for a department, for a given publication year, with respect to articles published in level 2 journals is equal to the sum of the department author fractions over the articles (published in the year) multiplied by 3 , the weight for a an article published in a level 2 journal (Table 1).

In ABM, each considered UU department is assigned a publication score for each considered publication year. In the application of the Norwegian model, the publication period is 2011-2018.

\subsection{Field normalized citation impact}

An important principle in evaluative bibliometrics is to compare like with like. It is a fact that citation volumes are not equal across research fields. For instance, the volumes are considerably larger in biomedicine than in pure mathematics. This is due to different citation practices in the two fields. If publications from fields as these two should be compared with regard to citation impact, some form of normalization of raw citations scores should be performed. In ABM, all used citation impact indicators are field normalized.

The publication period used for the citation analyses is 2010-2017. The end publication year in the version of CWTS Monitor used is 2017. Further, a later end year than 2017 would not be proper due to an inappropriately short citation window.

ABM makes use of two kinds of field normalized citation indicators: publication-level indicators and journal-level indicators. In the next section, we treat the former ones, whereas the latter ones are treated in the section after the next section.

\section{Publication-level indicators}

Two publication-level indicators are used, namely mean normalized number of citations per publication (MNCS) and proportion of frequently cited publications (top 10\%) (PP(top 10\%)). These two indicators, which complements each other, are used, for instance, in the CWTS Leiden Ranking 2019. ${ }^{3}$ For both MNCS and PP(top 10\%), and for the two journal-level indictors described below, a subject classification of publications is used for normalization purposes. The classification is generated by application of an advanced clustering methodology, in which a publication-publication relatedness measure based on direct citations between publications is utilized (Waltman \& van Eck, 2012). The classification, which contains about 4,000 classes, is such that each publication belongs to exactly one class. ${ }^{4}$

Regarding MNCS, and for each publication from a given department, the citation score of the publication is divided by the mean citation score across the publications, published in the same year as the publication, in the class to which the publication belongs. The resulting value is a (field) normalized

\footnotetext{
${ }^{3} \mathrm{http}: / /$ www.leidenranking.com/

${ }^{4}$ The traditional approach to field normalization typically uses the journal subject categories in WoS. However, one problem with the traditional approach is that the subfields of a certain field, where the fields are defined at a given level of granularity, might differ substantially from each other in terms of citation volume (e.g., van Eck et al., 2013). Moreover, it is clearly more reasonable to group the publications themselves into subject classes than to group the publications into such classes based on subject class membership of their journals.
} 
citation score (NCS) for the publication. The MNCS for the department is then obtained by dividing the weighted sum of NCS values over the publications from the department-where the weight of a publication consists of the author fraction the department has of the publication-by the sum of the department author fractions over the publications. If the department has a MNCS of, say, 1.2, the department performs $20 \%$ above world average $(=1)$.

For PP(top 10\%), and publication $i$ from the department, the same class in the classification is used as in the calculation of the NCS for $i$. The citation score distribution for the class, with respect to publications published the same year as $i$, is obtained, and the extent to which $i$ belongs to the $10 \%$ most frequently cited publications is determined. The result for $i$ is a value in the interval $[0,1]$, say (top $10 \%)_{i}{ }^{5} \mathrm{PP}($ top $10 \%$ ) for the department is then obtained by dividing the weighted sum of (top $10 \%$ ) values over the publications from the department-where the weight of a publication consists of the author fraction the department has of the publication-by the sum of the department author fractions over the publications (cf. the calculation of MNCS). If the department has a PP(top 10\%) value of, say, 8, the department performs $20 \%$ below the world average $(=10)$.

\section{Journal-level indicators}

The two publication-level citation indicators are complemented by two corresponding journal-level citation indicators, namely mean normalized journal impact score per publication (MNJS) and mean journal proportion of top 10\% publications (MJPP(top 10\%)).

For MNJS, each journal is first assigned an impact score, NJS, for each of the considered publication years. NJS, for given year, is equal to the mean across the NCSs of the publications in the journal published that year. Thus, NJS is a field normalized variant of the well-known Journal Impact Factor, values of which is available in Journal Citation Reports (provided by Clarivate Analytics). The latter indicator is not field normalized, however. Now, for a department and each publication from the department, the department author fraction of the publication is multiplied by the NJS of the journal of the publication with respect to the publication year of the publication. These operations yield a weighted sum over the publications from the department, and this sum is divided by the sum of the department author fractions over the publications. The result of the division is the MNJS for the department. Like MNCS, the world average for its journal-level counterpart, MNJS, is equal to 1.

The MJPP(top 10\%) indicator is calculated as follows. For each combination of a journal, a class, and a publication year, the proportion of the publications in that journal, class, and year that belong (to a degree in the interval $[0,1])$ to the $10 \%$ most frequently cited publications in that class and year is determined. This yields a PP(top 10\%) value for each combination of a journal, a class, and a year. This value is assigned to all publications in that journal, class, and year. In this way, each publication obtains a JPP(top 10\%) value. The MJPP(top 10\%) value of a department is obtained dividing the weighted sum of JPP(top 10\%) values over the publications from the department-where the weight of a publication consists of the author fraction the department has of the publication-by the sum of the department author fractions over the publications.

\subsection{Collaboration}

$\mathrm{ABM}$ uses two collaboration indicators: proportion of international collaborative publications ( $\mathrm{PP}(\mathrm{int}$ collab)) and proportion of publications with industry (PP(industry)). PP(int collab) for a department is the proportion of its publications that have been co-authored with two or more countries, whereas $\mathrm{PP}$ (industry) for a department is the proportion of its publications that have been co-authored with one or more industrial organizations. All private sector for profit business enterprises, covering all manufacturing and services sectors, are regarded as industrial organizations.

\footnotetext{
${ }^{5}$ The approach to assign fractions of publications to the (for instance) $10 \%$ most frequently cited publications is described and discussed by Waltman and Schreiber (2013). The approach has the property to produce exactly $10 \%$ top $10 \%$ publications. In Appendix 2, we show how the extent to which publication $i$ belongs to the $10 \%$ most frequently cited publications is calculated.
} 
Here, and normally, whole counts (and not fractional counts) are used for collaboration analysis. This means, taken international collaboration as an example, that if an UU department publication has at least one foreign address, relative to Sweden, the department is assigned one international collaborative publication, regardless of the author fraction the department has of the publication. If fractional counts are used, collaboration might be underestimated. However, publications with many organizations, like some of the publications in certain subfields of physics, do not indicate, to any larger extent, collaboration between the involved organizations.

For the collaboration part of ABM, the publication period is 2010-2017.

\subsection{Open access}

Open access (OA) in the simplest explanation is the concept of publications being available freely online without any costs or other access barriers to the reader. In the research policy bill "Collaborating for knowledge" (Prop. 2016/17:50), the Government presents a national goal that all scientific publications that are the result of publicly funded research should be published immediately open access, and it is stated that it is a shared responsibility for all actors in the research system to strive to achieve this goal. Also large national, and international, funders such as the Swedish Research Council and Horizon 2020 (European Commission) mandate OA publishing. This makes it relevant to measure the trend of OA publishing at UU.

There are different ways for a researcher to achieve OA publication and many different ways to define or source a publication as being OA. In ABM, we use information from DiVA, DOAJ and WoS as primary sources to distinguish between the different classes of OA. Gold OA is defined as a publication made freely available online by the publisher through its own services. Gold hybrid OA is defined as a publication published in a traditional subscription-based journal that is immediately freely available online through the publisher. In ABM, Gold hybrid is a subset of Gold OA. Green OA is defined as any publication available freely online through other services than the ones provided by the journal itself, often a postprint in an institutional repository. In $\mathrm{ABM}$, a publication $P$ is considered to be open access if at least one of the following four conditions is satisfied:

1) The full text of $P$ is published OA in DiVA

2) $P$ is included in a journal classified as OA journal in DiVA

3) $P$ is included in a journal classified as OA journal in DOAJ

4) $P$ is classified as "gold" or "green" OA in $\mathrm{WoS}^{6}$

For the OA part of ABM, the publication period is 2011-2018.

\section{Results}

In this section, we report the results of ABM. We first give, as a background, some results for UU as a whole. We then report the results for the UU departments, grouped by scientific domain.

Each of the sections 3.2-3.4, which corresponds to scientific domains, initially puts forward tables, which correspond to faculties and which report publication volumes (absolute and relative) and WoS coverage by department and publication type. Regarding WoS coverage, the validity of bibliometric indicators are in general higher if the coverage is high compared to low. Within a given section corresponding to a scientific domain, like Section 3.2, the other results are grouped based on what is analyzed: publishing volume and publishing level (the Norwegian model), field normalized citation impact, collaboration, and OA. Within a group of the indicated kind, the results are grouped by faculty.

\footnotetext{
${ }^{6}$ WoS classify OA through a third party service, Unpaywall https:/clarivate.com/webofsciencegroup/solutions/open-access/
} 


\subsection{UU as a whole}

In Figure 1, an overview of the subject profile of UU is given. ${ }^{7}$ In the map of the figure, all WoS subject categories in which UU has at least one publication of the types "Article" or "Review" and published in the period 2008-2017 are represented (237 categories). The size of a node indicates the publication output of UU in the corresponding category, whereas the color of a node indicates citation impact, relative to the world average, of the publications in the category. Citation impact is measured by the indicator MNCS. The color coding is as follows:

- Blue: The impact of a university in a subject category is far below world average

- Green: The impact of a university in a subject category is around world average.

- Red: The impact of a university in a subject category is far above world average.

Observe that the number of publications in some subject categories is very low. In such cases, the color of the category should be interpreted with caution.

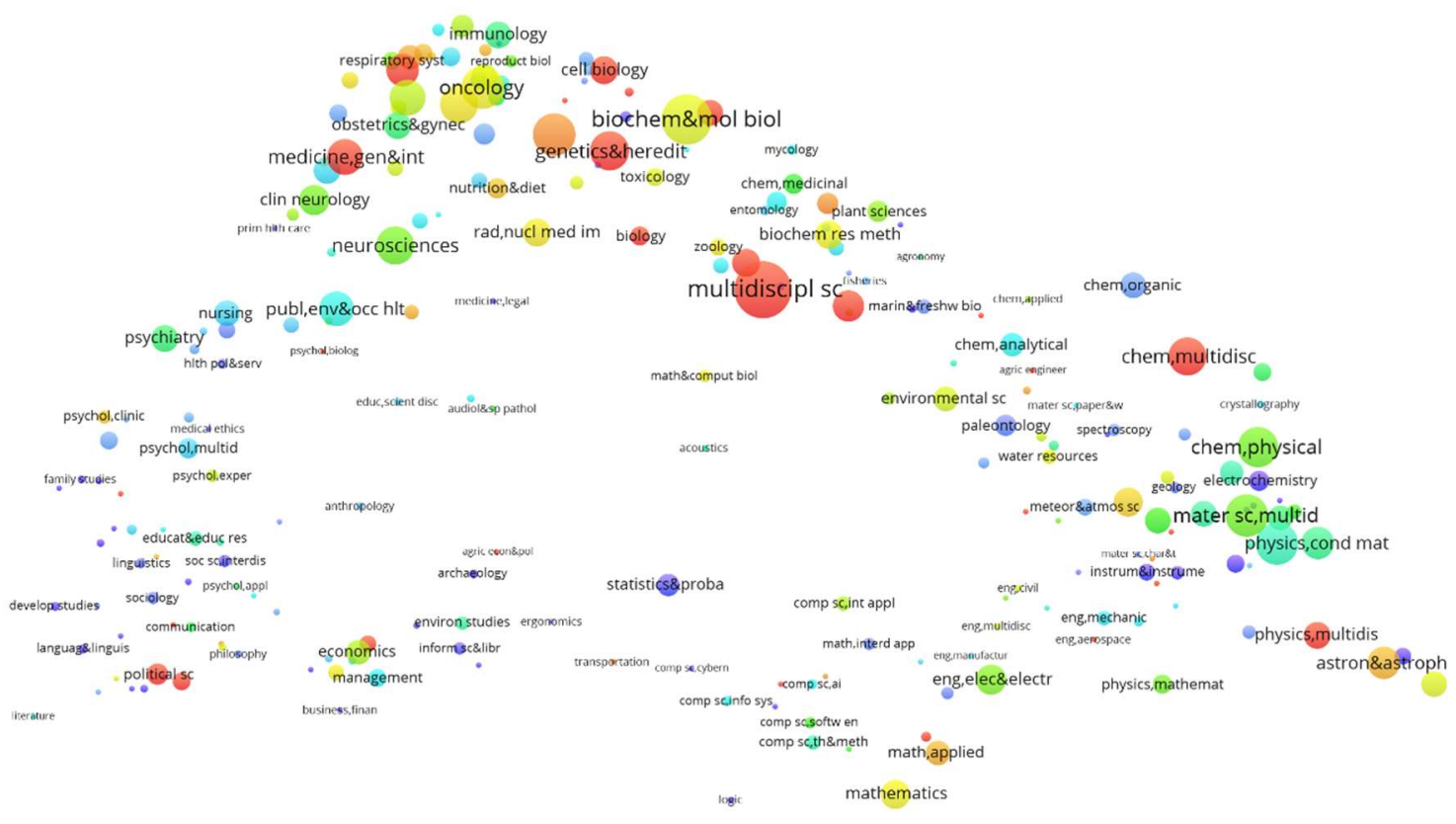

\& vosviewer

Figure 1. Subject profile map for UU. Publication period: 2008-2017.

In Figure 2, UUs proportion (in \%) of the total number of publication fractions (P) for UU in combination with 10 benchmarking universities (see Table 2) is shown by publication year. The figure also shows UUs proportion of the total number of publication fractions belonging to the top $10 \%$ most frequently cited ( $\mathrm{P}($ top $10 \%)$ ) for the same combination, where class (and thereby subject) and publication year of the publications are taken into account. The figure can be interpreted as the research production of UU over time, relative to a comparable set of universities.

\footnotetext{
${ }^{7}$ The subject profile overview was inspired by the CWTS web page at the following address: https://www.vosviewer.com/university-profile-maps.
} 


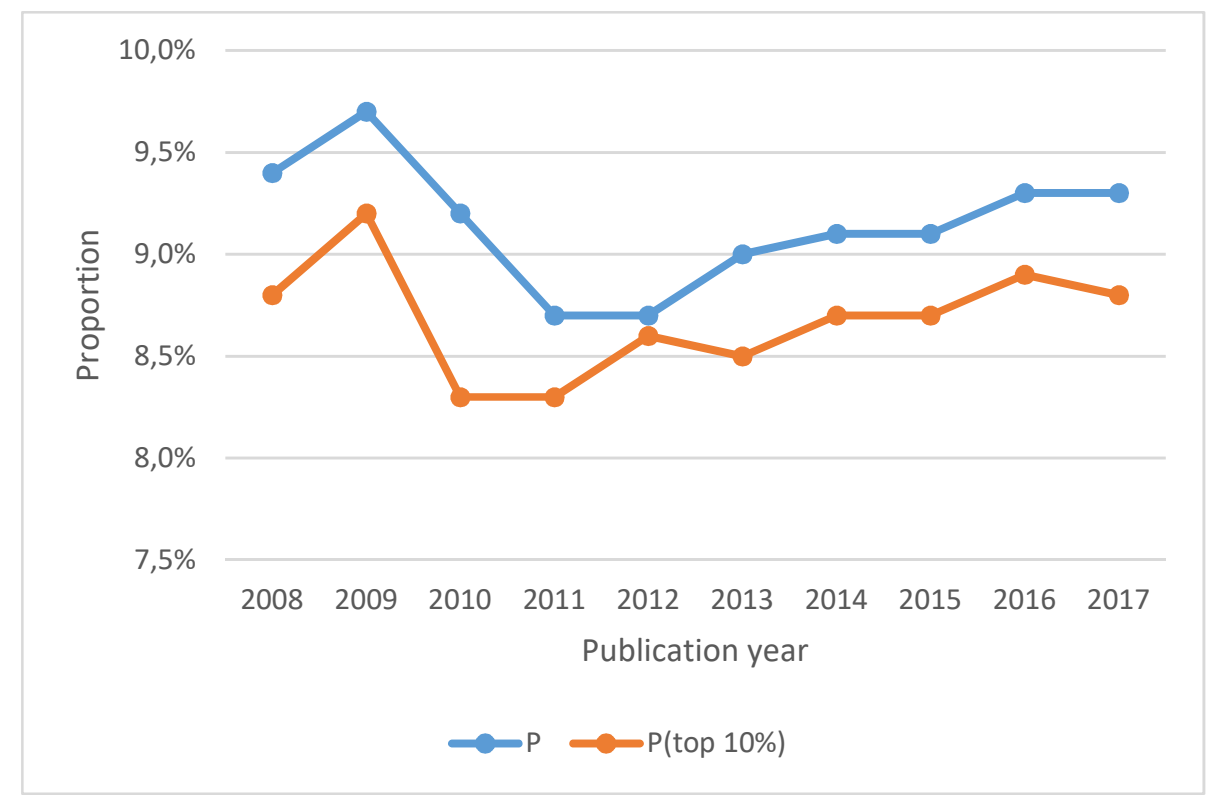

Figure 2. UUs proportion of publication fractions and of publication fractions belonging to the top $10 \%$ most frequently cited, by publication year and with regard to UU in combination with 10 benchmarking universities. Publication period: 2008-2017.

In Table 2, the 10 benchmarking universities are listed, together with a brief motivation for their inclusion.

Table 2. The 10 benchmarking universities.

\begin{tabular}{ll}
\hline Benchmark university & Brief motivation for inclusion \\
\hline Durham University & European reference, belonging to the Matariki ${ }^{8}$ network \\
Eberhard Karls University Tübingen & European reference, belonging to the Matariki and Guild ${ }^{9}$ networks \\
Ghent University & European reference, belonging to U4Society ${ }^{10}$ and Guild \\
Lund University & Swedish reference, belonging to the SLUG ${ }^{11}$ network \\
Stockholm University & Swedish reference, belonging to the SLUG network \\
University of Copenhagen & Nordic reference \\
University of Gothenburg & Swedish reference, belonging to the SLUG network \\
University of Groningen & European reference, belonging to U4Society and Guild \\
University of Helsinki & Nordic reference \\
University of Oslo & Nordic reference \\
\hline
\end{tabular}

In Figure 3, UUs proportion in different OA-classes compared to the total production is shown. We count the total production as articles, reviews, books, book chapters and conference proceedings. Full counts are used. For Uppsala University as a whole, the proportion of OA publishing is increasing during the entire period, but are well below 40\% during the entire period even though the large Swedish and European funders mandate immediate OA. The major part of the increase is Gold Hybrid publications.

\footnotetext{
${ }^{8}$ The Matariki network is an international group of seven universities.

${ }^{9}$ The Guild is a network of 21 European universities.

${ }^{10}$ U4Society is a collaboration between five European universities.

${ }^{11}$ SLUG is a Swedish network of the Stockholm, Lund, Uppsala and Gothenburg universities.
} 


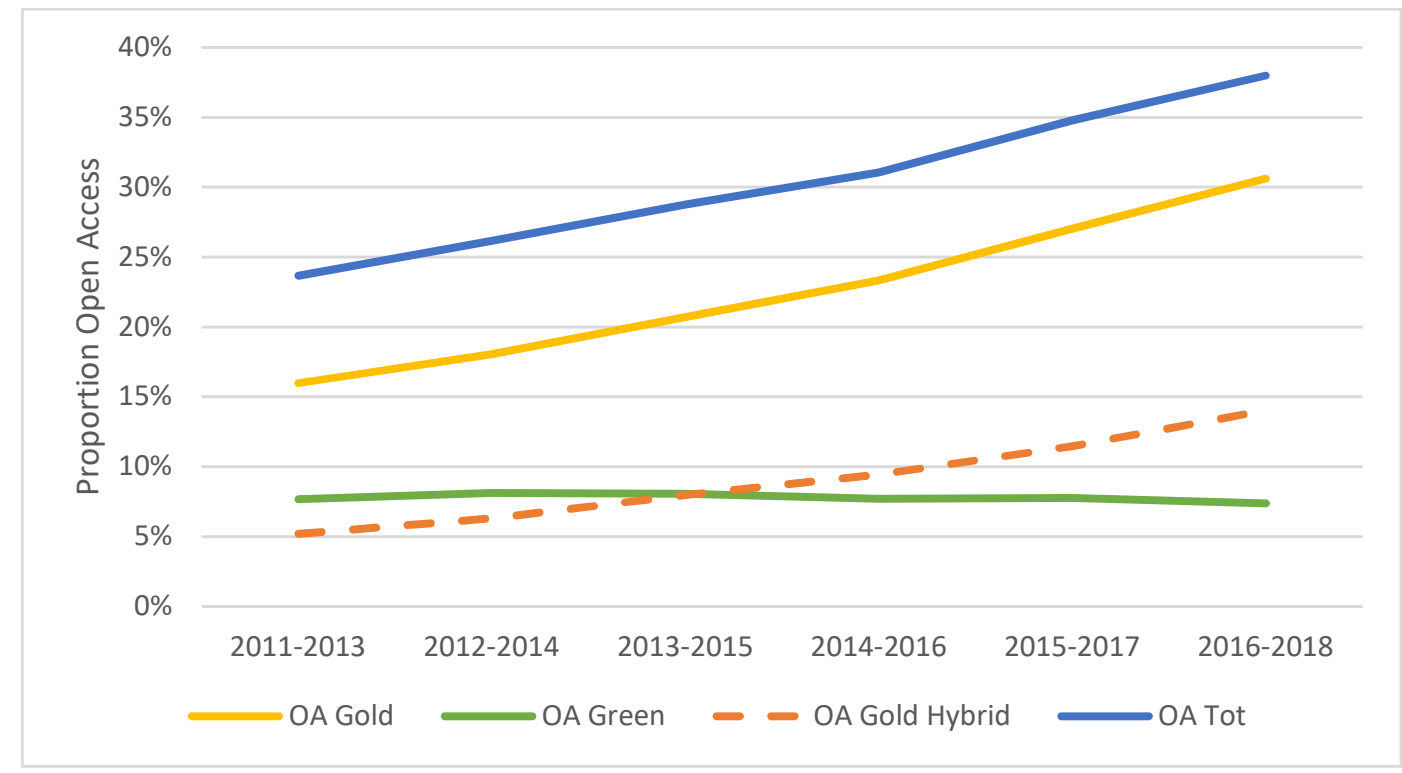

Figure 3. UUs proportion of different OA-classes compared to the total scientific production of UU. Publication period: 2011-2018. 3-year moving average.

\subsection{HumSam}

The number of HumSam units of analysis included in ABM is 31:28 departments and three faculties. Faculty of Law and Faculty of Theology consists of only one large department each, responsible for all publications. Nearly all the publications of the Faculty of Education belong to the Department of Education, and the few remaining ones belong to SWEDESD. For practical reasons, the faculty is therefore regarded as one unit in the ABM.

Tables 3-6 report publication volume in terms of both fractional counts and full counts (within parentheses) by department (by faculty for Table 5) and publication type, and WoS coverage. WoS coverage concerns the number of WoS publications of the four types represented in the tables relative to all publications of these types for the department.

Table 3. Faculty of Arts. Publication volume by publication type, and WoS coverage (in \%). Publication period: 2010-2018. Data source: GLIS.

\begin{tabular}{lcccccc}
\hline Department & Article & $\begin{array}{c}\text { Article in } \\
\text { anthology }\end{array}$ & $\begin{array}{c}\text { Publication type } \\
\text { Monograph }\end{array}$ & $\begin{array}{c}\text { Conference } \\
\text { paper }\end{array}$ & Total & $\begin{array}{c}\text { WoS } \\
\text { coverage }\end{array}$ \\
\hline ABM & $91,6(120)$ & $51,8(60)$ & $5,8(7)$ & $19,3(26)$ & $168,5(213)$ & 26,9 \\
Archeology and & & & & & & \\
Ancient History & $221,2(306)$ & $239,4(262)$ & $16,7(21)$ & $39,1(48)$ & $516,4(637)$ & 14,6 \\
Art History & $67,1(85)$ & $96,9(102)$ & $10,8(13)$ & $17,0(25)$ & $191,8(225)$ & 13,1 \\
Cultural & & & & & & \\
Anthropology and & & & & & \\
Ethnology & $117,8(133)$ & $113,7(127)$ & $26,4(32)$ & $6,3(6)$ & $264,2(298)$ & 6,9 \\
Game Design & $10,9(16)$ & $1,3(2)$ & $50,0(50)$ & $14,8(23)$ & $77,0(91)$ & 5,0 \\
Gender Research & $130,4(177)$ & $131,3(157)$ & $13,4(18)$ & $15,6(20)$ & $290,7(372)$ & 14,8 \\
History & $186,7(205)$ & $283,8(288)$ & $37,2(43)$ & $33,4(36)$ & $541,1(572)$ & 12,4 \\
History of Sciences & & & & & & \\
and Ideas & $150,3(161)$ & $135,7(147)$ & $15,2(19)$ & $1,0(1)$ & $302,1(328)$ & 10,7 \\
Literature & $187,3(201)$ & $283,9(297)$ & $71,5(74)$ & $6,6(8)$ & $549,2(580)$ & 1,9 \\
Musicology & $110,0(116)$ & $68,0(70)$ & $10,0(10)$ & $2,5(3)$ & $190,5(199)$ & 10,3 \\
Philosophy & $160,1(170)$ & $138,0(140)$ & $12,8(15)$ & $10,5(11)$ & $321,5(336)$ & 18,6 \\
\hline
\end{tabular}


Table 4. Faculty of Languages. Publication volume by publication type, and WoS coverage (in \%). Publication period: 2010-2018. Data source: GLIS.

\begin{tabular}{|c|c|c|c|c|c|c|}
\hline \multirow[t]{2}{*}{ Department } & \multicolumn{6}{|c|}{ Publication type } \\
\hline & Article & $\begin{array}{l}\text { Article in } \\
\text { anthology }\end{array}$ & Monograph & $\begin{array}{c}\text { Conference } \\
\text { paper }\end{array}$ & Total & $\begin{array}{c}\text { WoS } \\
\text { coverage }\end{array}$ \\
\hline English & $123,1(131,0)$ & $101,4(108)$ & $15,7(17)$ & $5,5(6)$ & $245,6(262)$ & 23,0 \\
\hline $\begin{array}{l}\text { Linguistics } \\
\text { and Philology } \\
\text { Modern }\end{array}$ & $319,5(369)$ & $274,7(301)$ & $61,3(70)$ & $130,1(194)$ & $785,7(934)$ & 8,6 \\
\hline $\begin{array}{l}\text { Languages } \\
\text { Scandinavian }\end{array}$ & $255,8(275)$ & $225,9(233)$ & $20,5(26)$ & $37,9(41)$ & $540,1(575)$ & 7,7 \\
\hline Languages & $427,6(460)$ & $214,4(231)$ & $25,8(31)$ & $72,5(79)$ & $740,4(801)$ & 2,3 \\
\hline
\end{tabular}

Table 5. Faculty of Education, Faculty of Law and Faculty of Theology. Publication volume by publication type, and WoS coverage (in \%). Publication period: 2010-2018. Data source: GLIS.

\begin{tabular}{lcccccc}
\hline Faculty & Article & $\begin{array}{c}\text { Publication type } \\
\text { Anticle in } \\
\text { anthology }\end{array}$ & $\begin{array}{c}\text { Monograph } \\
\text { Conference } \\
\text { paper }\end{array}$ & Total & $\begin{array}{c}\text { WoS } \\
\text { coverage }\end{array}$ \\
\hline $\begin{array}{l}\text { Faculty of } \\
\text { Education }\end{array}$ & $309,6(442)$ & $281,7(358)$ & $46,4(68)$ & $52,1(70)$ & $689,8(938)$ & 14,6 \\
$\begin{array}{l}\text { Faculty of Law } \\
\text { Faculty of }\end{array}$ & $537,8(583)$ & $516,1(537)$ & $123,8(140)$ & $11,1(13)$ & $1188,5(1273)$ & 3,1 \\
Theology & $356,1(389)$ & $456,4(477)$ & $77,0(89)$ & $19,1(23)$ & $908,6(978)$ & 8,8 \\
\hline
\end{tabular}

Table 6. Faculty of Social Sciences. Publication volume by publication type, and WoS coverage (in \%). Publication period: 2010-2018. Data source: GLIS.

\begin{tabular}{|c|c|c|c|c|c|c|}
\hline \multirow[t]{2}{*}{ Department } & \multicolumn{6}{|c|}{ Publication type } \\
\hline & Article & $\begin{array}{l}\text { Article in } \\
\text { anthology }\end{array}$ & Monograph & $\begin{array}{c}\text { Conference } \\
\text { paper }\end{array}$ & Total & $\begin{array}{c}\text { WoS } \\
\text { coverage }\end{array}$ \\
\hline $\begin{array}{l}\text { Business Studies } \\
\text { Russian and }\end{array}$ & $292,2(409)$ & $275,1(348)$ & $53,9(75)$ & $155,0(195)$ & $776,2(1027)$ & 17,6 \\
\hline Eurasian Studies & $243,1(282)$ & $153,1(166)$ & $22,4(25)$ & $9,8(11)$ & $428,4(484)$ & 15,2 \\
\hline Economic History & $121,9(150)$ & $242,2(267)$ & $33,8(40)$ & $29,0(35)$ & $427,0(492)$ & 11,8 \\
\hline Economics & $164,2(290)$ & $18,0(23)$ & $6,1(9)$ & $1,0(1)$ & $189,3(323)$ & 68,1 \\
\hline $\begin{array}{l}\text { Food Studies, } \\
\text { Nutrition and }\end{array}$ & & & & & & \\
\hline Dietetics & $67,4(133)$ & 45,4 (49) & $1,0(3)$ & $7,1(11)$ & $120,9(196)$ & 35,4 \\
\hline $\begin{array}{l}\text { Government } \\
\text { Housing and Urban }\end{array}$ & $336,0(452)$ & $266,5(300)$ & $33,5(40)$ & $38,0(42)$ & $674,0(834)$ & 27,5 \\
\hline $\begin{array}{l}\text { Research } \\
\text { Informatics and }\end{array}$ & $175,2(293)$ & $158,6(200)$ & $18,5(25)$ & $82,7(97)$ & $435,0(615)$ & 19,0 \\
\hline $\begin{array}{l}\text { Media } \\
\text { Peace and Conflict }\end{array}$ & $125,0(173)$ & $104,5(126)$ & $14,7(18)$ & $111,9(151)$ & $356,1(458)$ & 17,7 \\
\hline Studies & $241,3(293)$ & $180,4(206)$ & $24,3(29)$ & $39,0(42)$ & $484,9(570)$ & 31,5 \\
\hline Psychology & $354,7(689)$ & $35,7(47)$ & $3,0(5)$ & $18,1(26)$ & $411,5(767)$ & 70,5 \\
\hline $\begin{array}{l}\text { Social and } \\
\text { Economic }\end{array}$ & & & & & & \\
\hline Geography & $152,5(213)$ & $58,6(69)$ & 7,7 (12) & $20,2(24)$ & $238,9(318)$ & 44,4 \\
\hline Sociology & $237,6(324)$ & $147,1(188)$ & $29,9(43)$ & $66,3(79)$ & $480,9(634)$ & 23,4 \\
\hline Statistics & $53,6(114)$ & $1,5(2)$ & $1,0(2)$ & $0,5(1)$ & $56,6(119)$ & 86,8 \\
\hline
\end{tabular}


3.2.1 Publishing volume and publishing level-The Norwegian model

Faculty of Arts

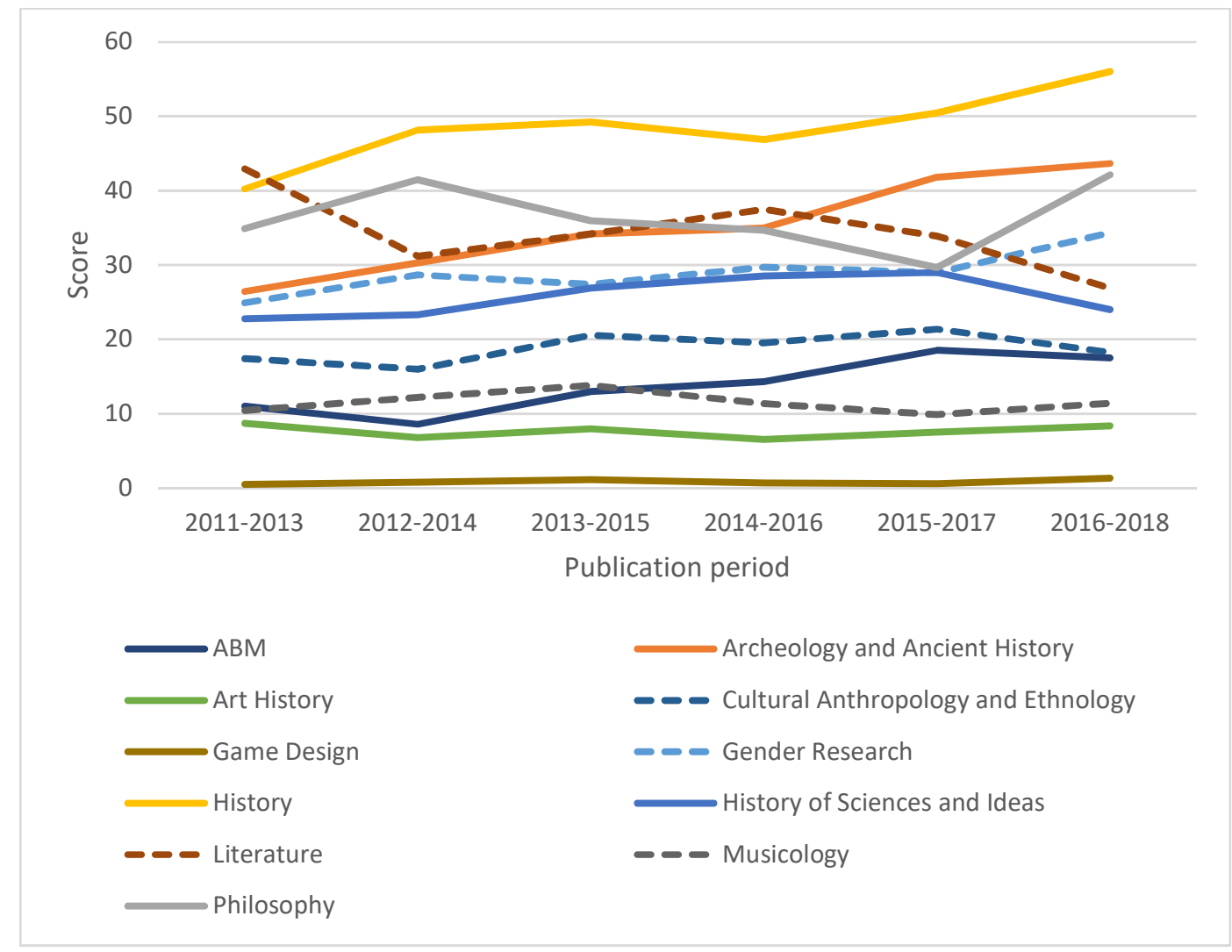

Figure 4. Faculty of Arts. Norwegian score by publication period. 3-year moving average.

Table 7. Faculty of Arts. Proportion (in \%) publication fractions at level 2 relative to the sum of publication fractions across levels 1 and 2 (given within parentheses) by publication period. 3-year moving average.

\begin{tabular}{lcccccc}
\hline Department & & & & & & \\
& $\mathbf{2 0 1 1 - 2 0 1 3}$ & $\mathbf{2 0 1 2 - 2 0 1 4}$ & $\mathbf{2 0 1 3 - 2 0 1 5}$ & $\mathbf{2 0 1 4 - 2 0 1 6}$ & $\mathbf{2 0 1 5 - 2 0 1 7}$ & $\mathbf{2 0 1 6 - 2 0 1 8}$ \\
\hline ABM & $39,9(23,4)$ & $26,8(19,9)$ & $42,9(24,7)$ & $32,9(29,1)$ & $37,2(31,4)$ & $32,3(31,7)$ \\
Archeology & & & & & & \\
and Ancient & & & & & & \\
History & $27,7(65,6)$ & $33,2(71,6)$ & $31,9(90,1)$ & $36,1(88,4)$ & $40,4(94,1)$ & $47,7(88,6)$ \\
Art History & $1,4(23,7)$ & $8,6(19,9)$ & $6,7(23,7)$ & $8,3(19,0)$ & $3,6(21,9)$ & $12,6(20,5)$ \\
Cultural & & & & & & \\
Anthropology & & & & & & \\
and & & & & & \\
Ethnology & $33,0(31,8)$ & $33,0(31,8)$ & $37,8(38,3)$ & $38,0(40,0)$ & $38,2(45,0)$ & $27,9(46,5)$ \\
Game Design & $0,0(2,0)$ & $0,0(3,0)$ & $0,0(4,0)$ & $0,0(2,1)$ & $0,0(1,8)$ & $47,5(2,1)$ \\
Gender & & & & & & \\
Research & $37,8(65,0)$ & $31,0(61,4)$ & $23,1(60,0)$ & $20,0(63,8)$ & $29,3(60,0)$ & $29,6(73,3)$ \\
History & $23,7(92,8)$ & $19,6(104,5)$ & $23,9(104,1)$ & $32,3(92,9)$ & $36,1(95,5)$ & $41,9(98,1)$ \\
History of & & & & & & \\
Sciences and & & & & & \\
Ideas & $20,6(52,7)$ & $27,3(56,2)$ & $28,3(60,2)$ & $28,8(64,2)$ & $27,4(57,7)$ & $17,7(61,2)$ \\
Literature & $6,8(95,8)$ & $11,7(93,8)$ & $13,1(91,5)$ & $10,4(81,5)$ & $5,9(68,1)$ & $11,6(55,8)$ \\
Musicology & $35,1(18,5)$ & $16,7(30,0)$ & $14,2(35,2)$ & $12,8(31,2)$ & $21,3(28,2)$ & $32,8(29,0)$ \\
Philosophy & $32,5(80,0)$ & $39,4(91,3)$ & $36,0(76,3)$ & $45,6(76,8)$ & $50,0(58,0)$ & $51,0(74,5)$ \\
\hline
\end{tabular}


Faculty of Languages

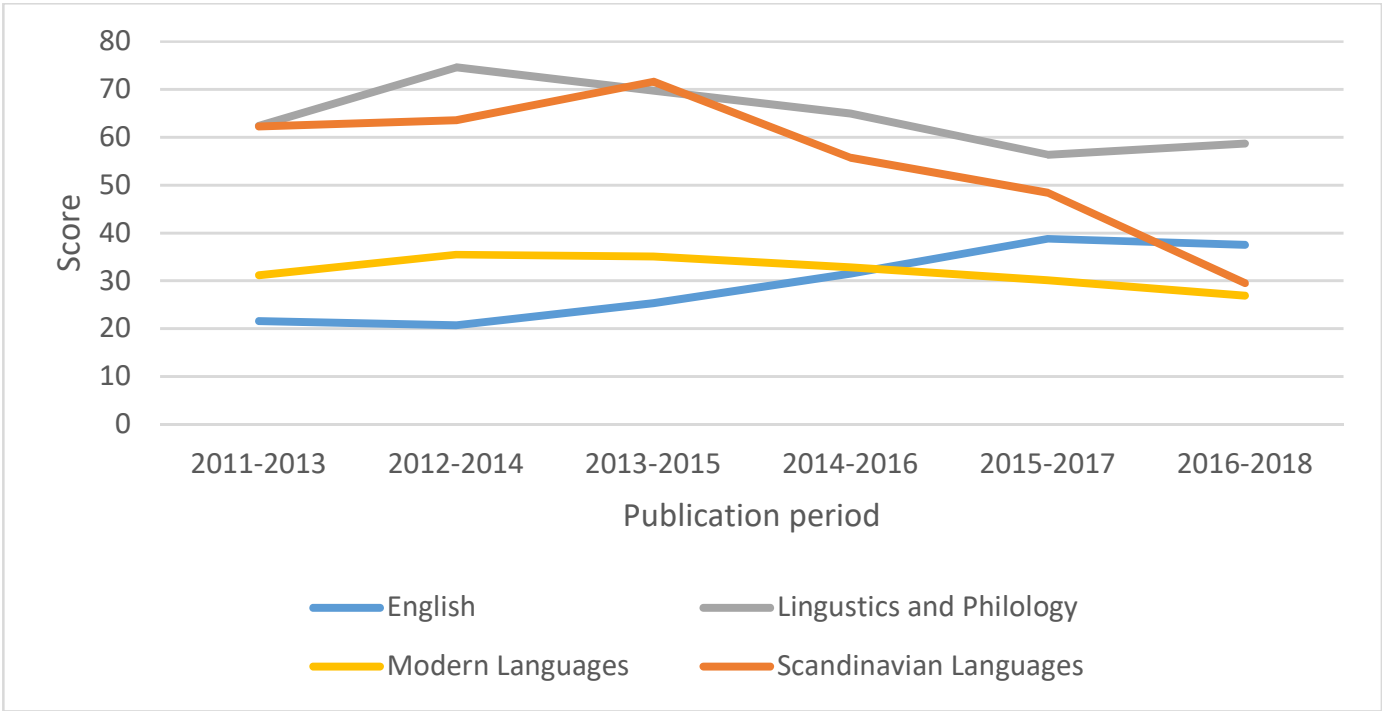

Figure 5. Faculty of Languages. Norwegian score by publication period. 3-year moving average.

Table 8. Faculty of Languages. Proportion (in \%) publication fractions at level 2 relative to the sum of publication fractions across levels 1 and 2 (given within parentheses) by publication period. 3-year moving average.

\begin{tabular}{lcccccc}
\hline Department & $\mathbf{2 0 1 1 - 2 0 1 3}$ & $\mathbf{2 0 1 2 - 2 0 1 4}$ & $\mathbf{2 0 1 3 - 2 0 1 5}$ & $\mathbf{2 0 1 4 - 2 0 1 6}$ & $\mathbf{2 0 1 5 - 2 0 1 7}$ & $\mathbf{2 0 1 6 - 2 0 1 8}$ \\
\hline $\begin{array}{l}\text { English } \\
\text { Linguistics } \\
\text { and Philology }\end{array}$ & $21,9(51,0)$ & $25,6(53,8)$ & $33,2(59,5)$ & $39,9(57,0)$ & $42,9(65,3)$ & $41,4(62,3)$ \\
$\begin{array}{l}\text { Modern } \\
\text { Languages }\end{array}$ & $16,9(122,9)$ & $53,2(135,5)$ & $52,7(129,9)$ & $55,6(110,7)$ & $47,5(101,4)$ & $53,5(105,2)$ \\
$\begin{array}{l}\text { Scandinavian } \\
\text { Languages }\end{array}$ & $28,4(121,6)$ & $15,9(99,3)$ & $16,4(96,8)$ & $15,2(92,0)$ & $18,4(80,8)$ & $13,1(83,8)$ \\
\hline
\end{tabular}


Faculty of Education, Faculty of Law and Faculty of Theology

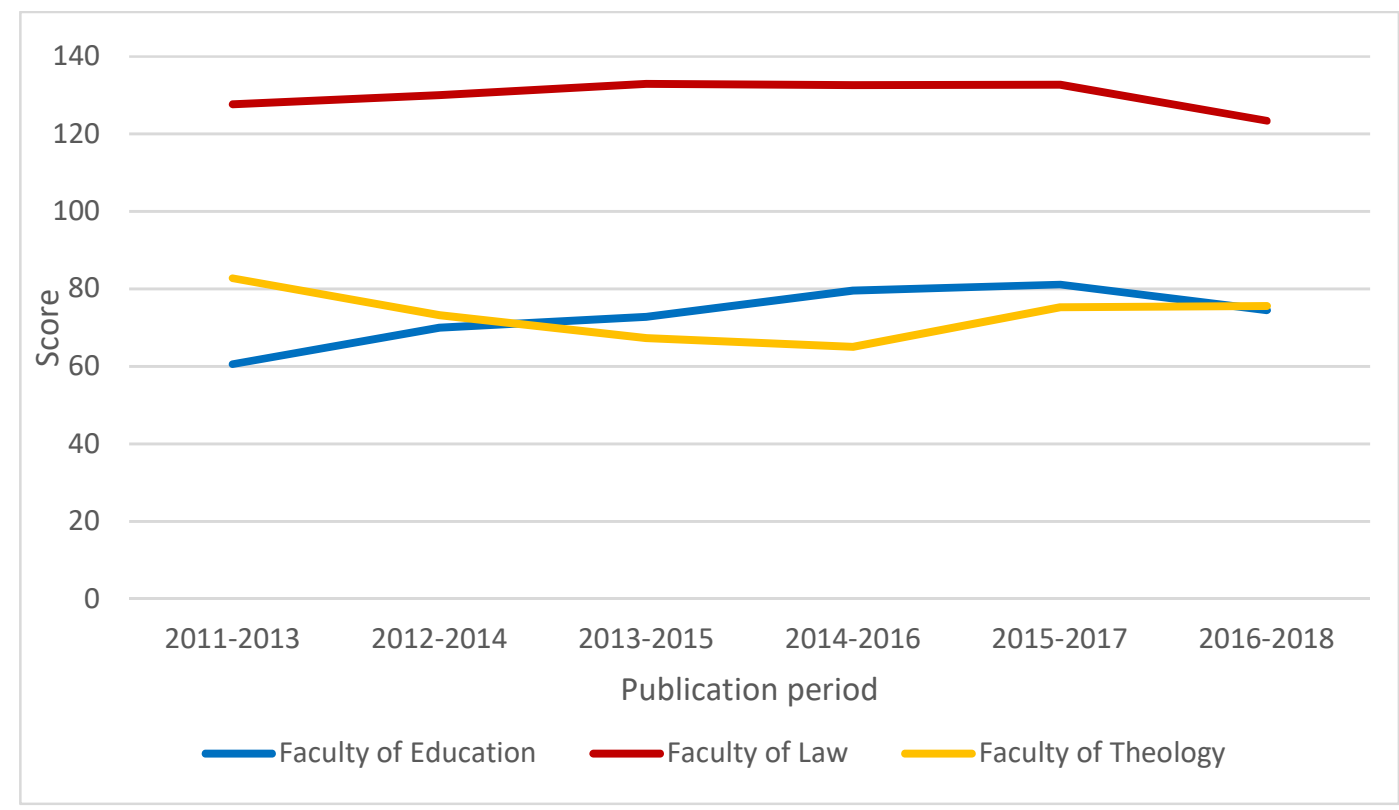

Figure 6. Faculty of Education, Faculty of Law and Faculty of Theology. Norwegian score by publication period. 3 -year moving average.

Table 9. Faculty of Education, Faculty of Law and Faculty of Theology. Proportion (in \%) publication fractions at level 2 relative to the sum of publication fractions across levels 1 and 2 (given within parentheses) by publication period. 3-year moving average.

\begin{tabular}{lcccccc}
\hline Department & $\mathbf{2 0 1 1 - 2 0 1 3}$ & $\mathbf{2 0 1 2 - 2 0 1 4}$ & $\mathbf{2 0 1 3 - 2 0 1 5}$ & $\mathbf{2 0 1 4 - 2 0 1 6}$ & $\mathbf{2 0 1 5 - 2 0 1 7}$ & $\mathbf{2 0 1 6 - 2 0 1 8}$ \\
\hline $\begin{array}{l}\text { Faculty of } \\
\text { Education }\end{array}$ & $10,4(129,6)$ & $11,7(137,0)$ & $16,1(142,8)$ & $19,5(164,6)$ & $25,4(159,0)$ & $27,8(136,3)$ \\
Faculty of & & & & & & \\
Law & $10,4(246,6)$ & $13,5(273,1)$ & $20,2(278,3)$ & $26,0(290,4)$ & $24,6(274,5)$ & $21,7(266,6)$ \\
$\begin{array}{l}\text { Faculty of } \\
\text { Theology }\end{array}$ & $28,3(177,8)$ & $33,4(156,3)$ & $35,1(148,6)$ & $33,7(139,0)$ & $32,3(159,8)$ & $39,0(152,1)$ \\
\hline
\end{tabular}




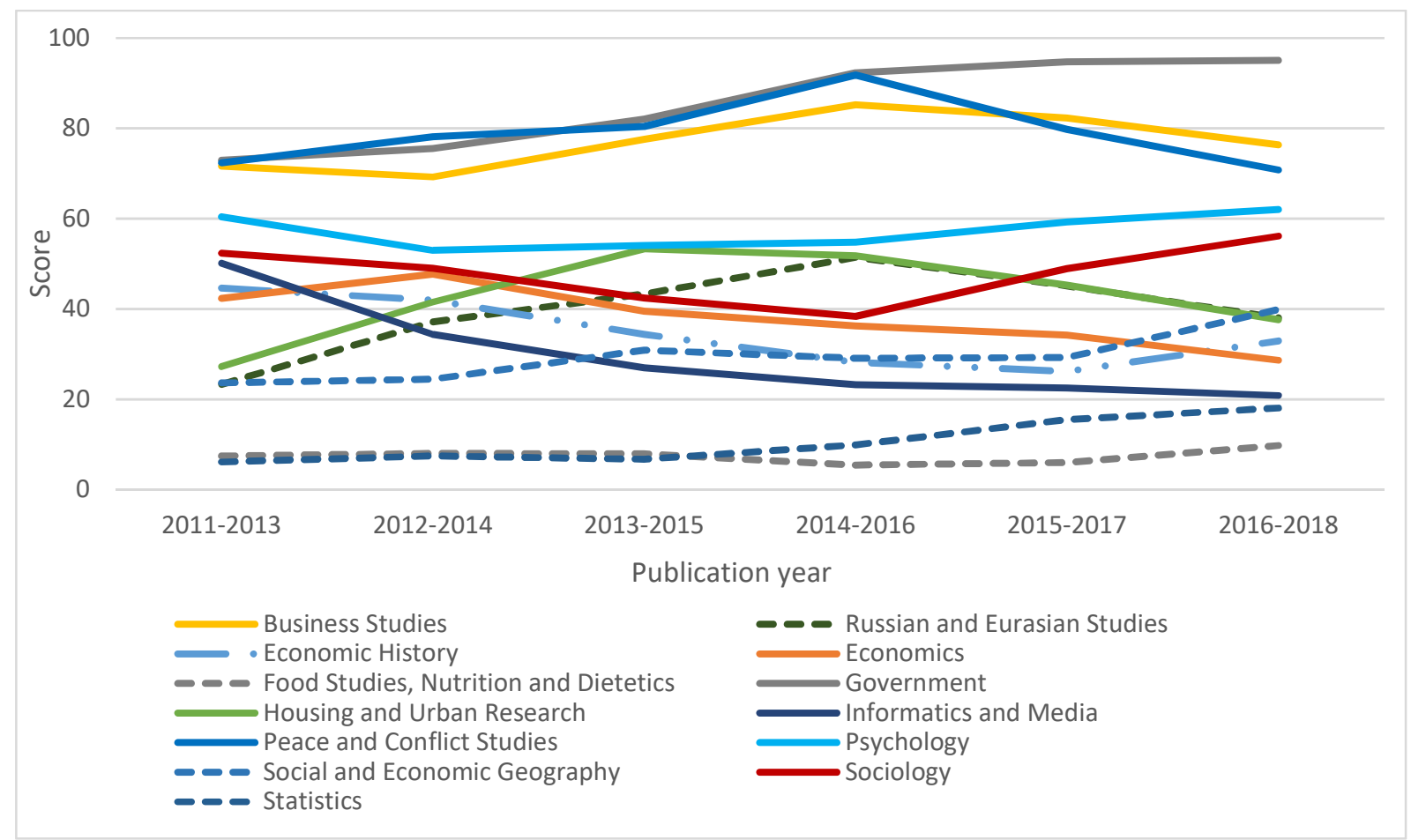

Figure 7. Faculty of Social Sciences. Norwegian score by publication period. 3-year moving average. 
Table 10. Faculty of Social Sciences. Proportion (in \%) publication fractions at level 2 relative to the sum of publication fractions across levels 1 and 2 (given within parentheses) by publication period. 3-year moving average.

\begin{tabular}{|c|c|c|c|c|c|c|}
\hline \multicolumn{7}{|l|}{ Department } \\
\hline \multicolumn{7}{|l|}{ Business } \\
\hline Studies & $31,8(144,3)$ & $34,5(131,2)$ & $31,0(142,7)$ & $33,8(164,6)$ & $36,4(159,7)$ & $37,3(155,8)$ \\
\hline \multicolumn{7}{|l|}{ Russian and } \\
\hline \multicolumn{7}{|l|}{ Eurasian } \\
\hline Studies & $25,2(49,5)$ & $21,8(69,0)$ & $33,3(84,1)$ & $31,8(108,4)$ & $34,1(99,3)$ & $28,6(78,5)$ \\
\hline \multicolumn{7}{|l|}{ Economic } \\
\hline History & $14,4(100,7)$ & $20,6(89,6)$ & $27,3(78,6)$ & $32,7(62,7)$ & $33,9(60,0)$ & $40,6(62,7)$ \\
\hline Economics & $32,1(71,5)$ & $38,3(74,2)$ & $44,3(57,4)$ & $46,3(53,5)$ & $51,0(48,9)$ & $50,3(42,9)$ \\
\hline \multicolumn{7}{|l|}{ Food Studies, } \\
\hline \multicolumn{7}{|l|}{ Nutrition and } \\
\hline Dietetics & $11,8(21,5)$ & $8,0(25,0)$ & $10,1(24,7)$ & $14,0(16,1)$ & $12,9(16,1)$ & $7,7(26,9)$ \\
\hline Government & $43,0(146,8)$ & $46,2(153,7)$ & $44,5(16,0)$ & $41,3(170,4)$ & $45,0(167,4)$ & $44,7(175,7)$ \\
\hline \multicolumn{7}{|l|}{ Housing and } \\
\hline \multicolumn{7}{|l|}{ Urban } \\
\hline Research & $25,7(62,9)$ & $37,4(81,2)$ & $33,7(105,4)$ & $31,3(100,5)$ & $30,7(92,6)$ & $31,2(75,6)$ \\
\hline \multicolumn{7}{|l|}{ Informatics } \\
\hline \multirow{2}{*}{\multicolumn{7}{|c|}{ Peace and }} \\
\hline & & & & & & \\
\hline \multicolumn{7}{|l|}{ Conflict } \\
\hline Studies & $42,5(135,9)$ & $46,8(144,5)$ & $51,3(125,6)$ & $57,1(141,3)$ & $54,3(131,6)$ & $58,4(130,2)$ \\
\hline Psychology & $28,9(117,4)$ & $21,9(113,7)$ & $21,6(115,3)$ & $23,9(116,0)$ & $25,4(124,4)$ & $29,1(122,9)$ \\
\hline \multicolumn{7}{|l|}{ Social and } \\
\hline \multicolumn{7}{|l|}{ Economic } \\
\hline Geography & $31,2(47,1)$ & $38,0(47,1)$ & $41,2(58,7)$ & $46,8(57,1)$ & $44,0(58,0)$ & $45,3(66,9)$ \\
\hline Sociology & $30,0(97,1)$ & $26,4(100,7)$ & $28,4(101,6)$ & $24,8(97,2)$ & $28,3(106,1)$ & $33,9(115,6)$ \\
\hline Statistics & $25,4(12,1)$ & $22,1(15,5)$ & $17,4(13,9)$ & $20,0(18,3)$ & $31,1(26,3)$ & $34,6(30,4)$ \\
\hline
\end{tabular}

\subsubsection{Field normalized citation impact}

\section{Faculty of Arts}

Table 11. Faculty of Arts. Publication fractions (P; full counts within parentheses), MNCS, PP(top 10\%), MNJS and MJPPtop10\% for the whole publication period 2010-2017.

\begin{tabular}{lccccc}
\hline Department & & \multicolumn{3}{c}{ Indicator } \\
& $\mathbf{P}$ & MNCS & PP(top 10\%) & MNJS & MJPPtop10\% \\
\hline ABM & $31,4(43)$ & 0,72 & 3,2 & 0,91 & 9,4 \\
Archeology and Ancient History & $30,0(70)$ & 0,98 & 8,4 & 0,91 & 10,2 \\
Art History & $3,2(9)$ & null & null & null & null \\
Cultural Anthropology and Ethnology & $7,5(11)$ & null & null & null & null \\
Game Design & $0,1(1)$ & null & null & null & null \\
Gender Research & $12,1(24)$ & null & null & null & null \\
History & $13,1(17)$ & null & null & null & null \\
History of Sciences and Ideas & $3,4(6)$ & null & null & null & null \\
Literature & $0,0(0)$ & null & null & null & null \\
Musicology & $0,7(2)$ & null & null & null & null \\
Philosophy & $24,1(27)$ & null & null & null & null \\
\hline
\end{tabular}




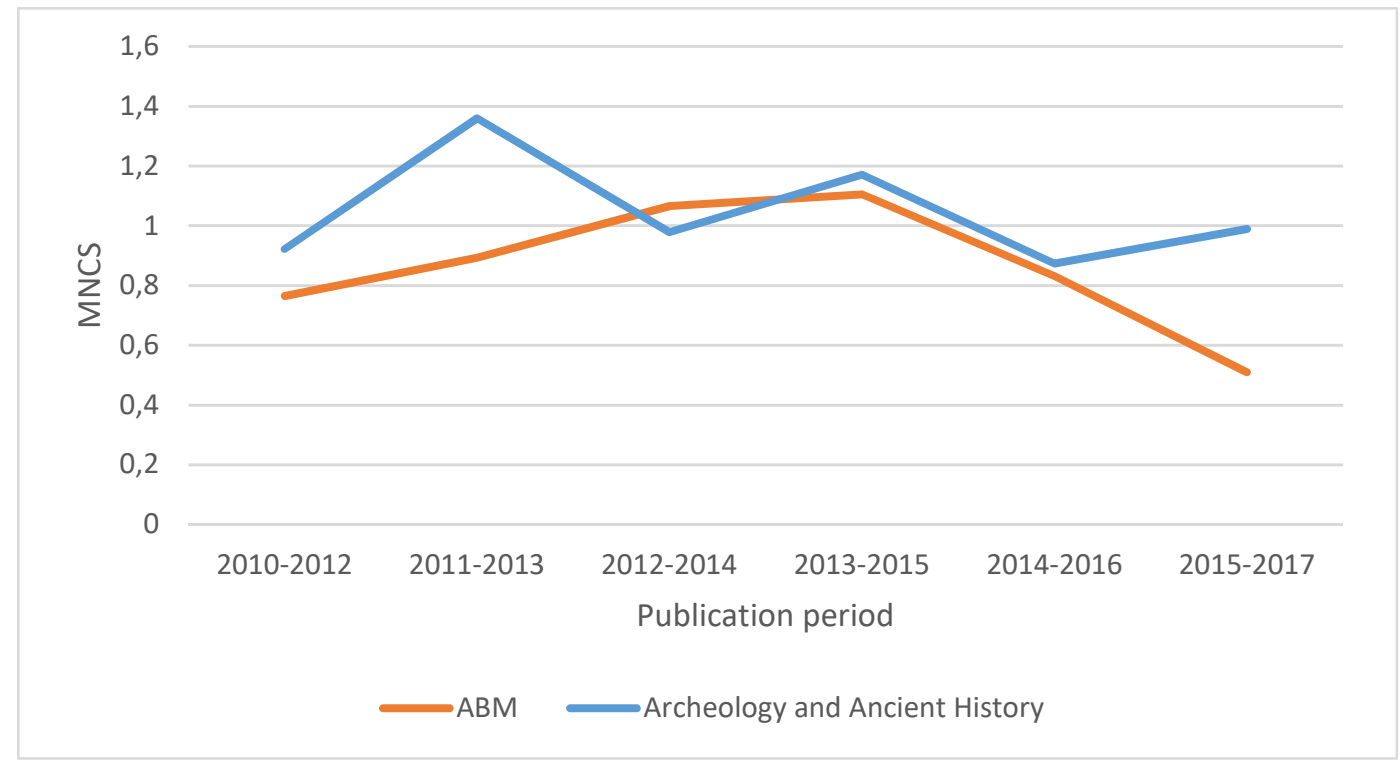

Figure 8. Faculty of Arts. MNCS by publication period. 3-year moving average.

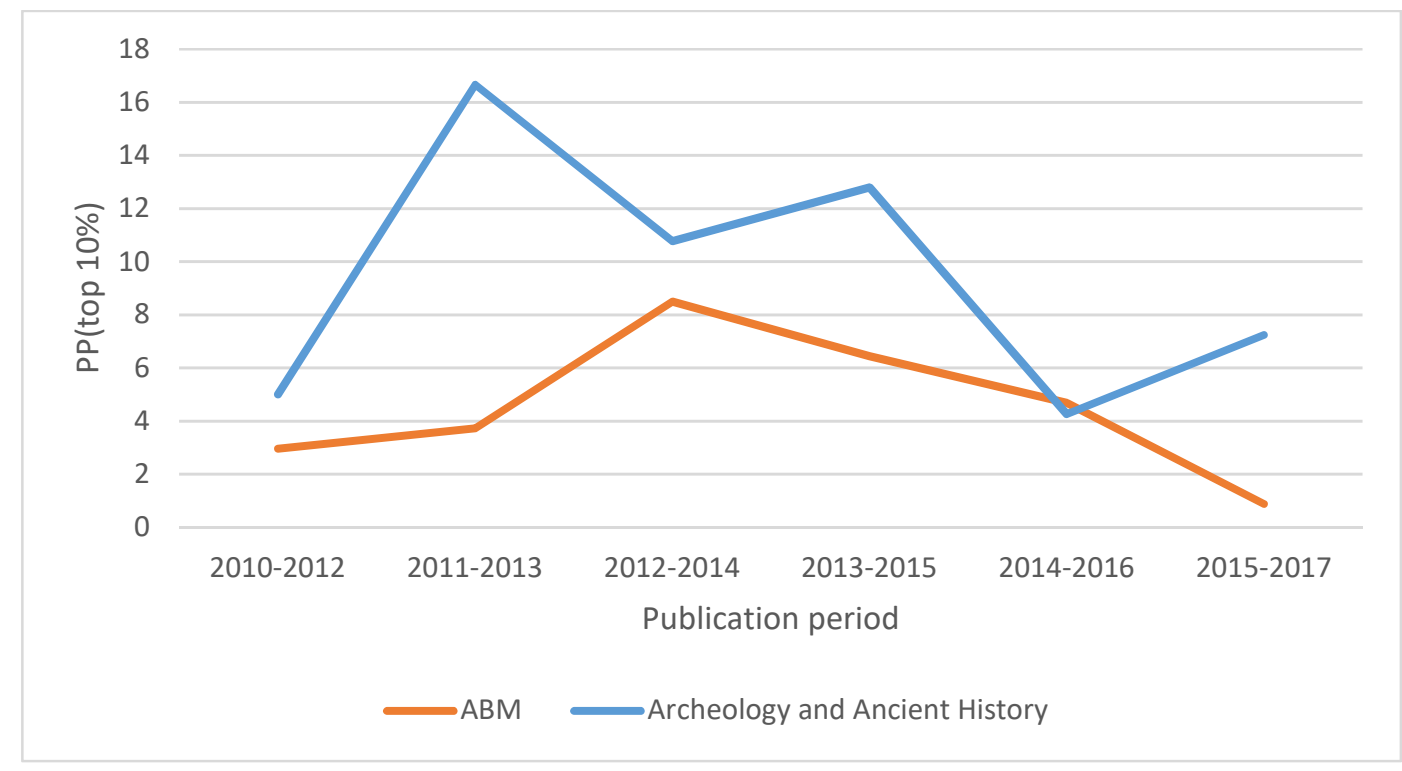

Figure 9. Faculty of Arts. PP(top 10\%) by publication period. 3-year moving average. 


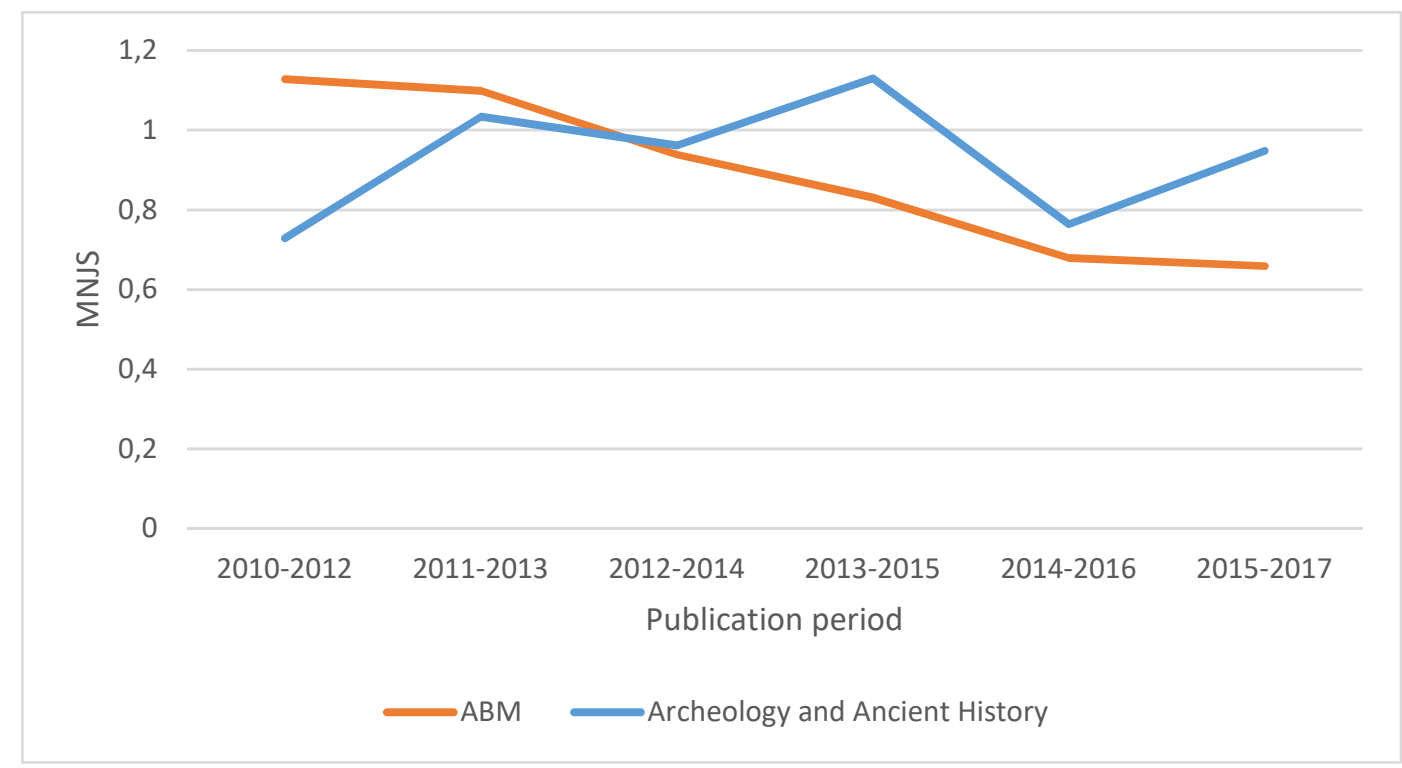

Figure 10. Faculty of Arts. MNJS by publication period. 3-year moving average.

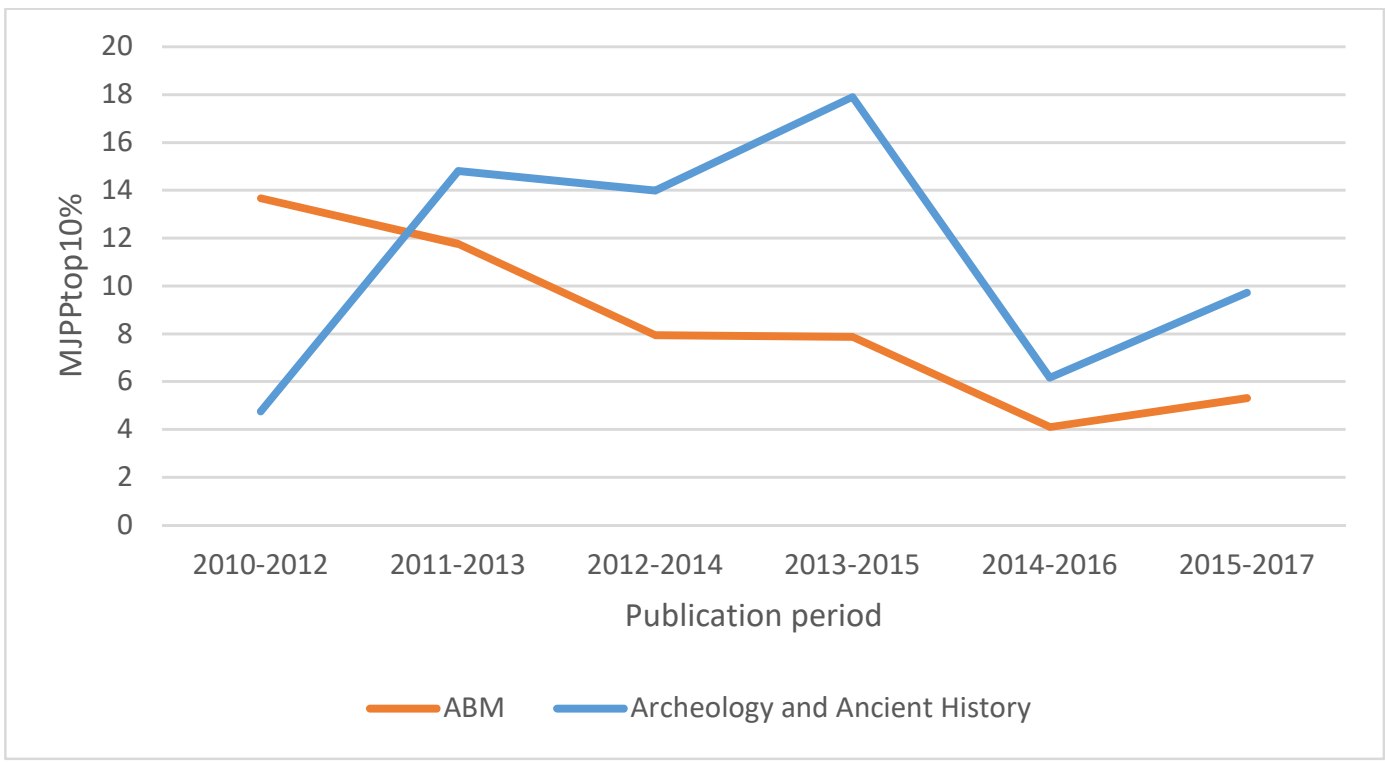

Figure 11. Faculty of Arts. MJPPtop10\% by publication period. 3-year moving average.

\section{Faculty of Languages}

Each of the four departments of the faculty has less than 40 publications for the publication period 2010-2017. This yields that the four departments are excluded from the citation analysis of ABM.

Table 12. Faculty of Languages. Publication fractions (P; full counts within parentheses), MNCS, PP(top 10\%), MNJS and MJPPtop10\% for the whole publication period 2010-2017.

\begin{tabular}{lccccc}
\hline Department & \multicolumn{5}{c}{ Indicator } \\
& P & MNCS & PP(top 10\%) & MNJS & MJPPtop10\% \\
\hline English & $8,5(9)$ & null & null & null & null \\
Linguistics and Philology & $16,2(30)$ & null & null & null & null \\
Modern Languages & $11,3(14)$ & null & null & null & null \\
Scandinavian Languages & $6,1(12)$ & null & null & null & null \\
\hline
\end{tabular}


Faculty of Education, Faculty of Law and Faculty of Theology

Table 13. Faculty of Education, Faculty of Law and Faculty of Theology. Publication fractions (P; full counts within parentheses), MNCS, PP(top 10\%), MNJS and MJPPtop10\% by department and for the whole publication period 2010-2017.

\begin{tabular}{lccccc}
\hline Department & \multicolumn{5}{c}{ Indicator } \\
& P & MNCS & PP(top 10\%) & MNJS & MJPPtop10\% \\
\hline Faculty of Education & $57,0(97)$ & 0,68 & 3,5 & 0,81 & 7,3 \\
Faculty of Law & $12,2(20)$ & null & null & null & null \\
Faculty of Theology & $11,8(23)$ & null & null & null & null \\
\hline
\end{tabular}

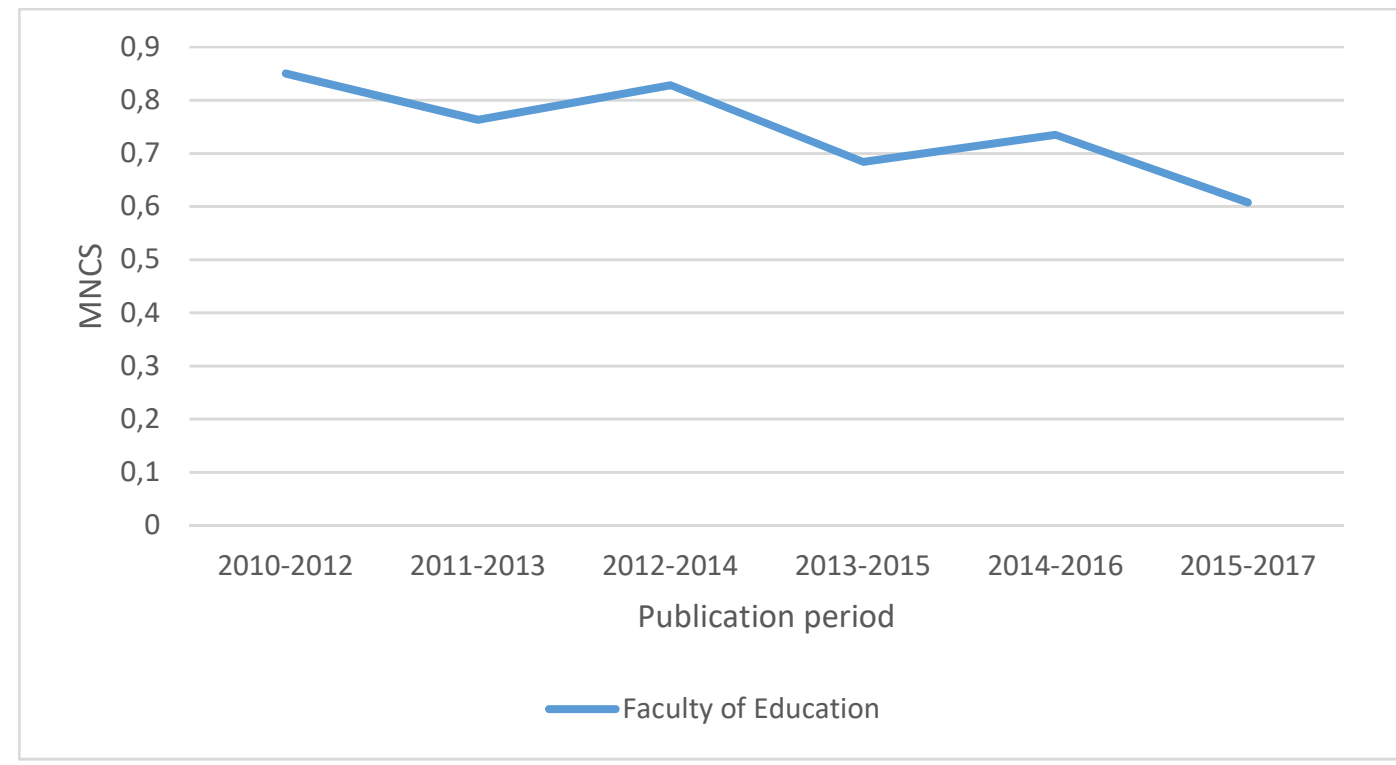

Figure 12. Faculty of Education. MNCS by publication period. 3-year moving average.

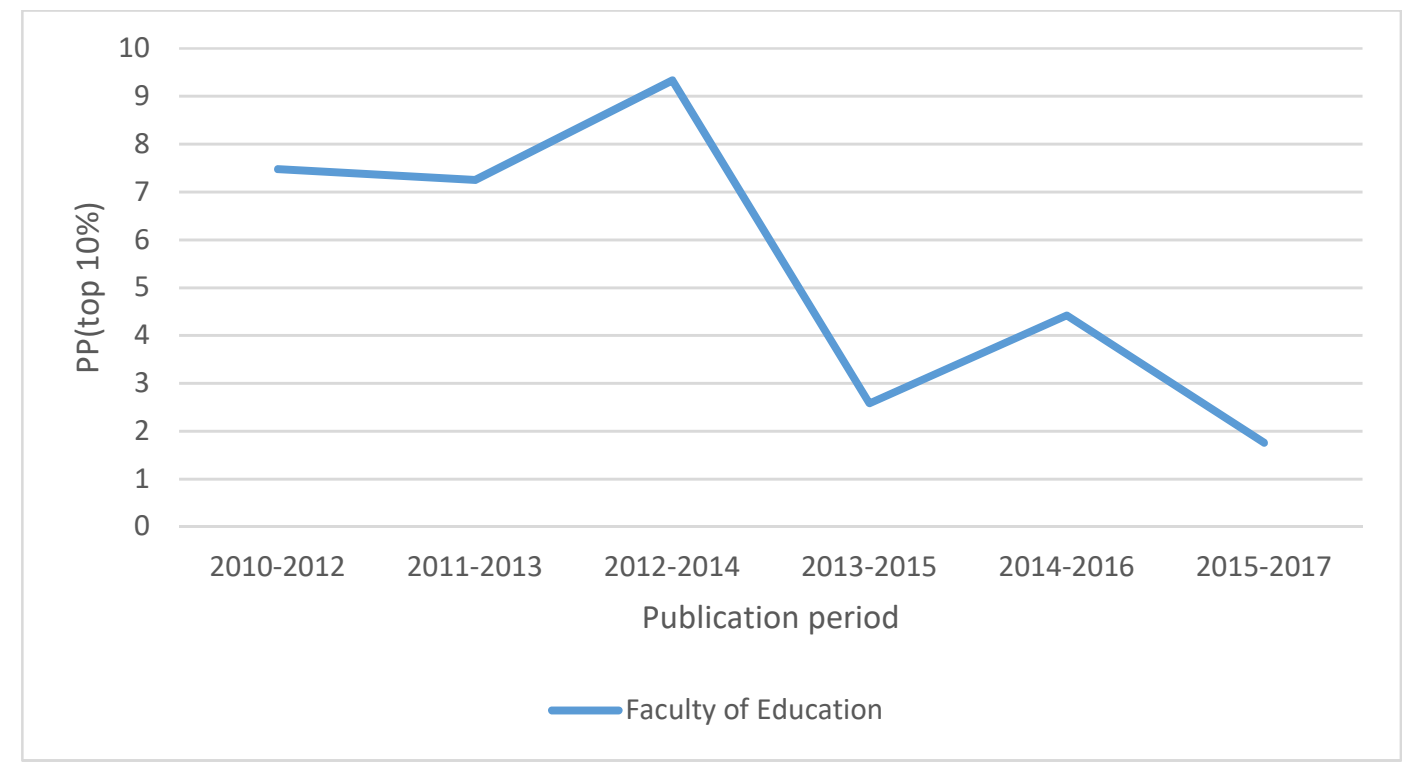

Figure 13. Faculty of Education. PP(top 10\%) by publication period. 3-year moving average. 


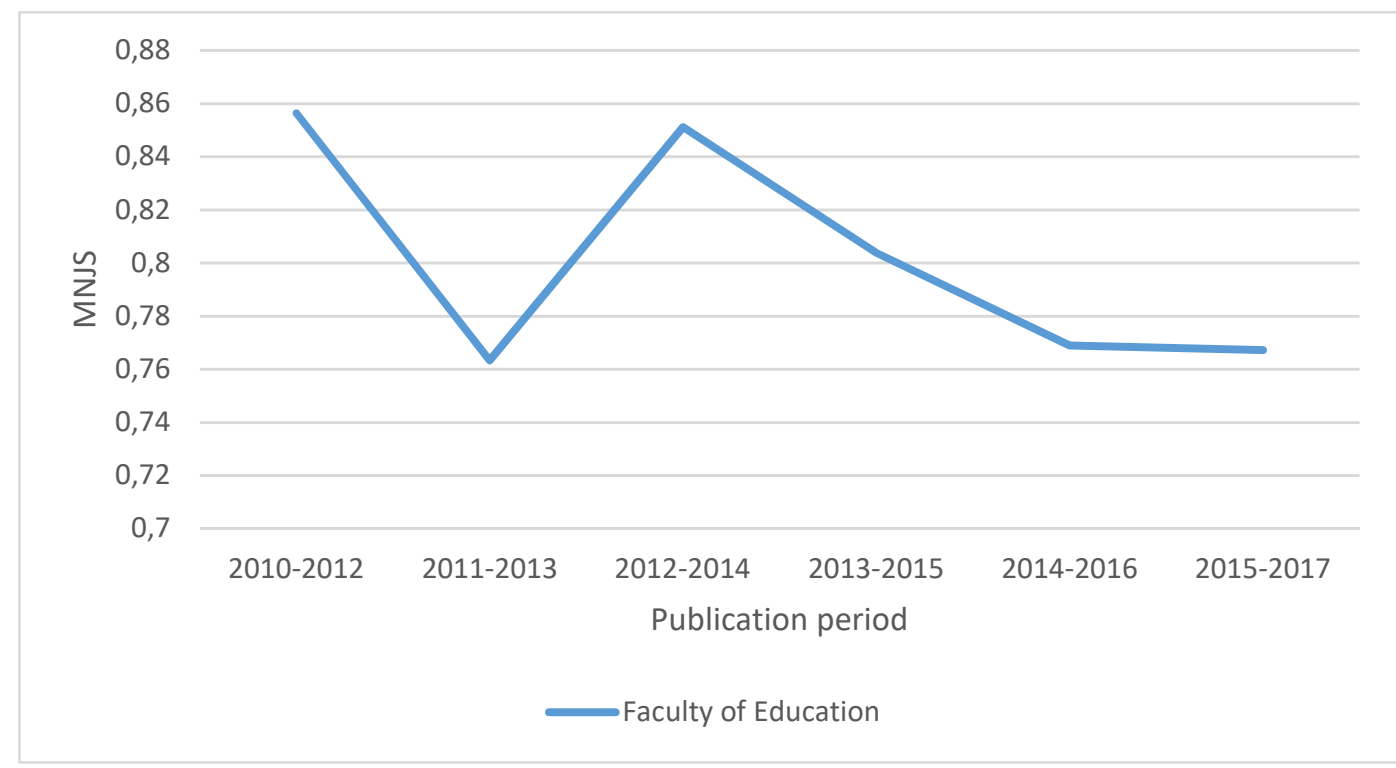

Figure 14. Faculty of Education. MNJS by publication period. 3-year moving average.

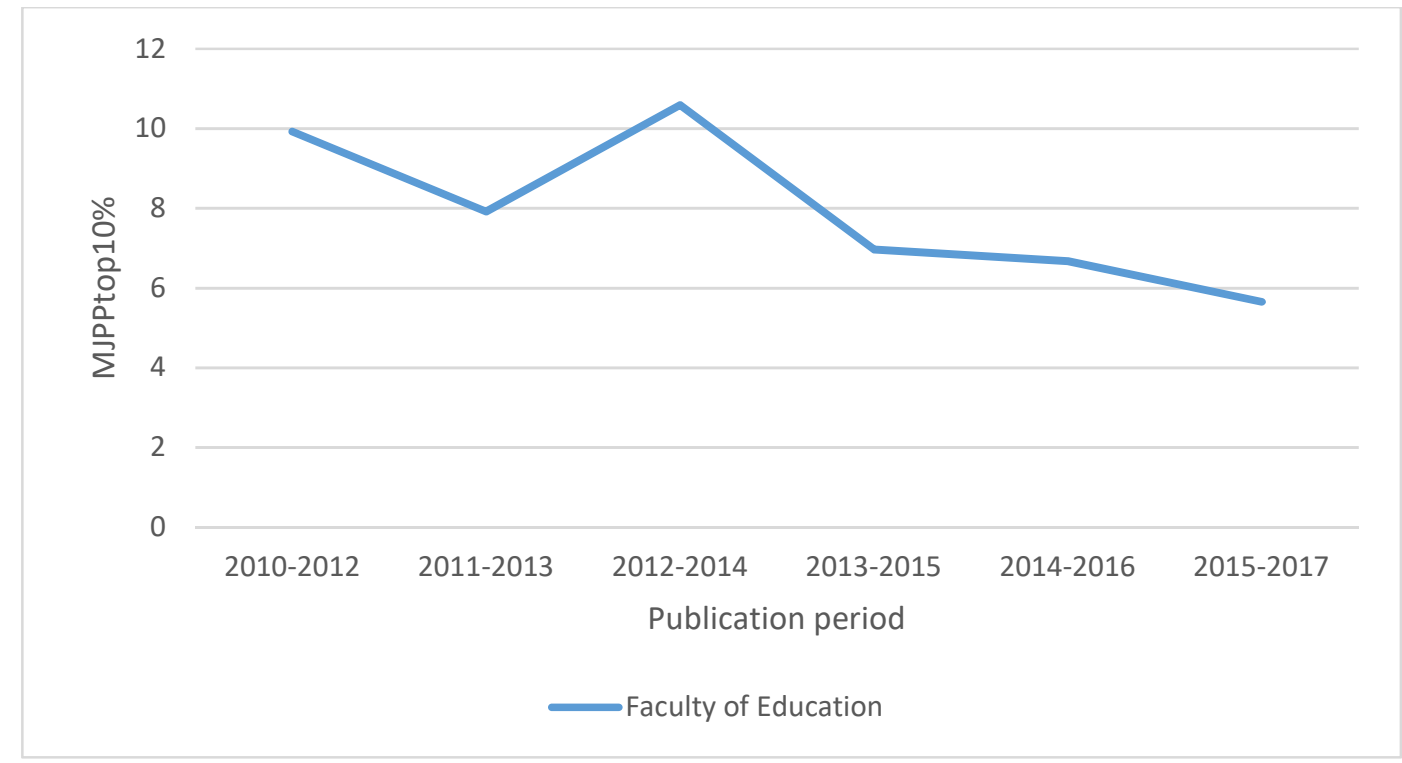

Figure 15. Faculty of Education. MJPPtop10\% by publication period. 3-year moving average.

\section{Faculty of Social Sciences}

Table 14. Faculty of Social Sciences. Publication fractions (P; full counts within parentheses), MNCS, PP(top $10 \%$ ), MNJS and MJPPtop10\% by department and for the whole publication period 2010-2017.

\begin{tabular}{lccccc}
\hline Department & P & MNCS & $\begin{array}{c}\text { Indicator } \\
\text { PP(top 10\%) }\end{array}$ & MNJS & MJPPtop10\% \\
\hline Business Studies & $83,1(146)$ & 0,98 & 10,0 & 1,02 & 9,9 \\
Russian and Eurasian Studies & $16,7(30)$ & null & null & null & null \\
Economic History & $17,7(29)$ & null & null & null & null \\
Economics & $85,6(184)$ & 1,03 & 11,0 & 1,07 & 11,4 \\
Food Studies, Nutrition and Dietetics & $28,7(69)$ & 0,57 & 4,0 & 0,75 & 7,3 \\
Government & $111,4(180)$ & 0,87 & 8,2 & 1,00 & 9,3 \\
Housing and Urban Research & $63,2(130)$ & 1,50 & 13,1 & 1,20 & 10,0 \\
Informatics and Media & $28,5(49)$ & 0,97 & 6,0 & 0,79 & 4,0 \\
Peace and Conflict Studies & $95,7(135)$ & 2,05 & 23,1 & 1,33 & 14,6
\end{tabular}


Psychology

Social and Economic Geography

Sociology

Statistics

$\begin{array}{ccccc}229,4(478) & 1,11 & 11,4 & 1,12 & 10,6 \\ 71,4(110) & 1,04 & 11,0 & 1,11 & 14,2 \\ 58,5(94) & 0,68 & 1,5 & 0,79 & 5,0 \\ 35,1(79) & 0,58 & 5,1 & 0,63 & 5,3\end{array}$

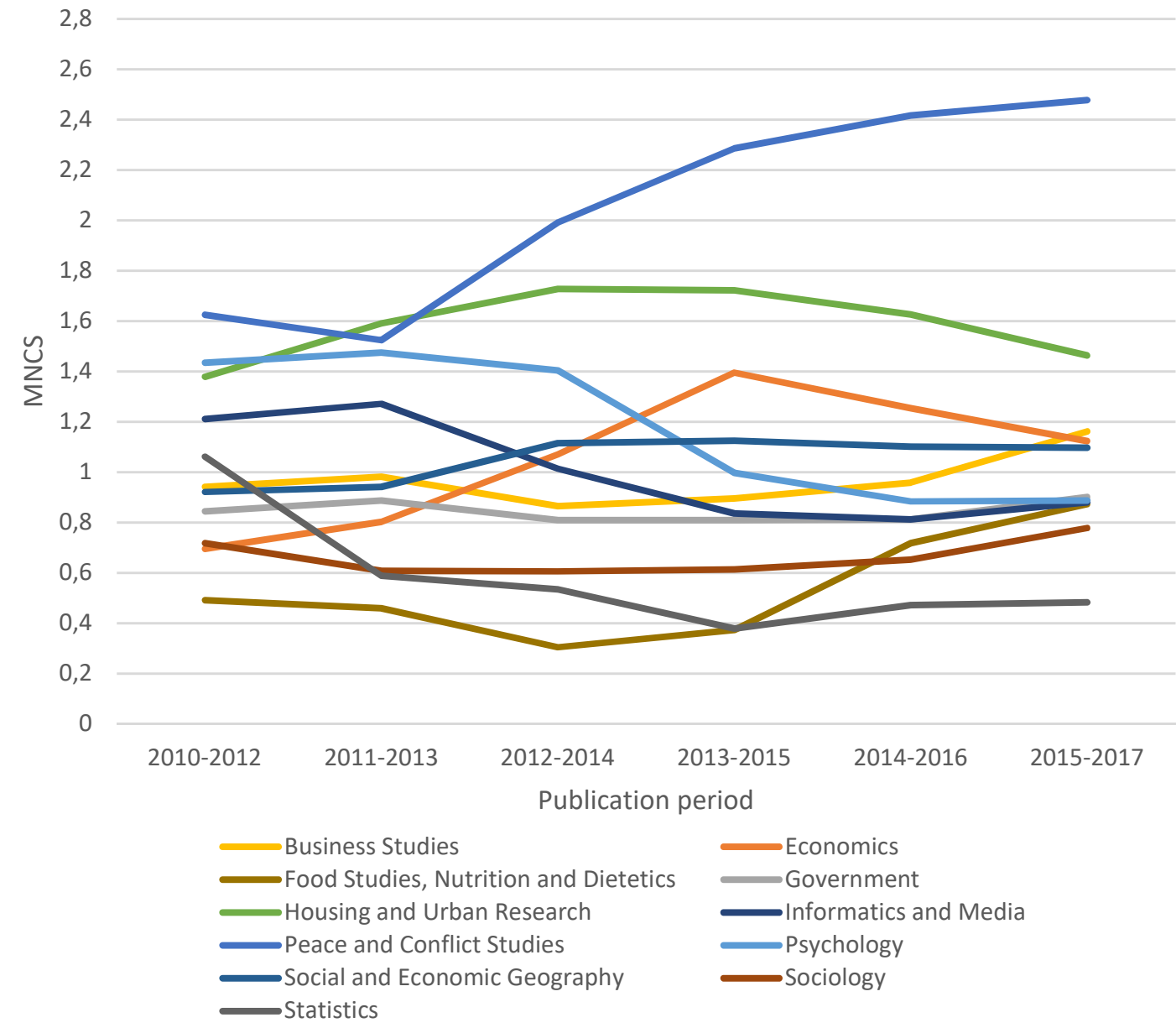

Figure 16. Faculty of Social Sciences. MNCS by publication period. 3-year moving average. 


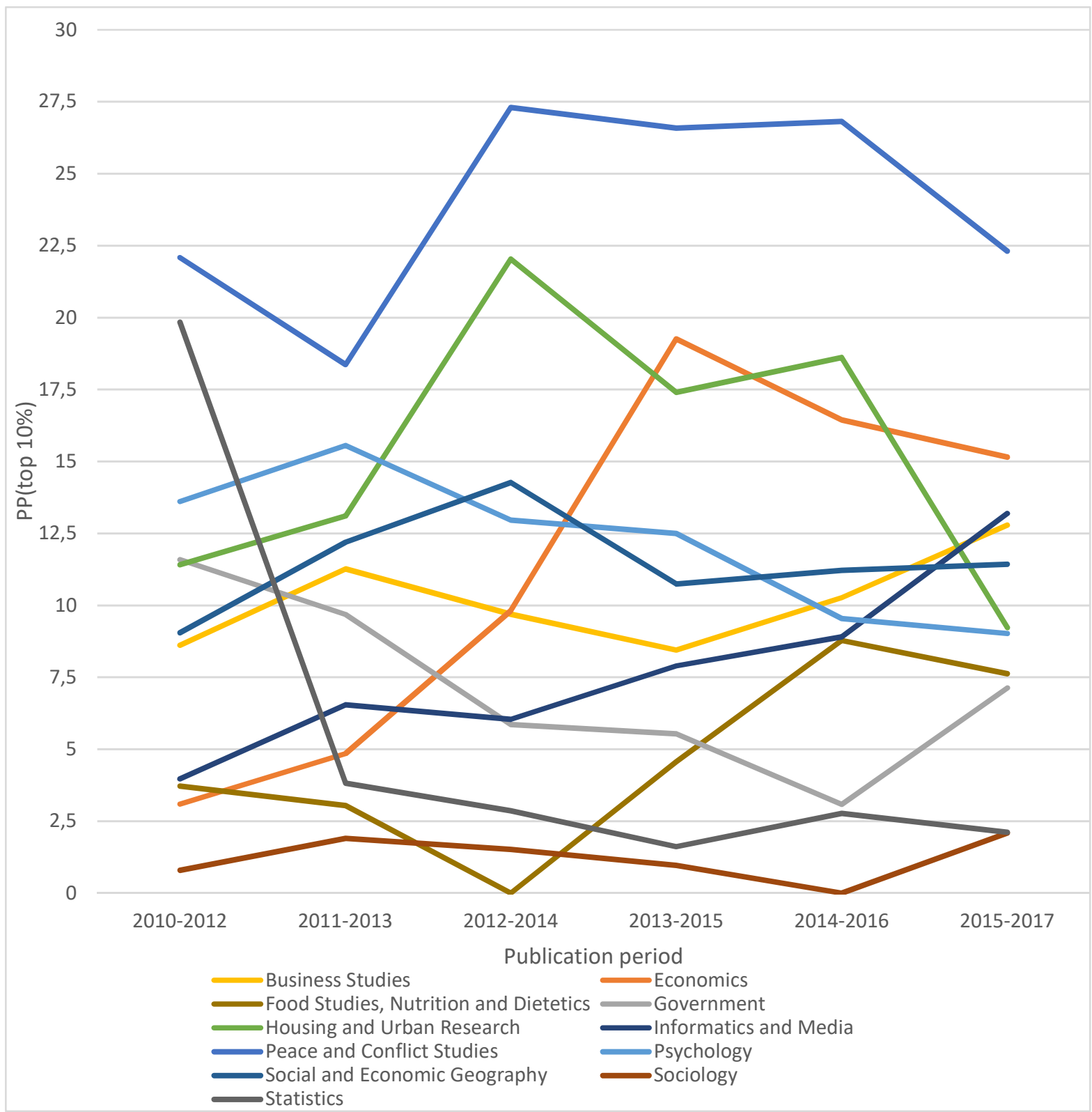

Figure 17. Faculty of Social Sciences. PP(top 10\%) by publication period. 3-year moving average. 


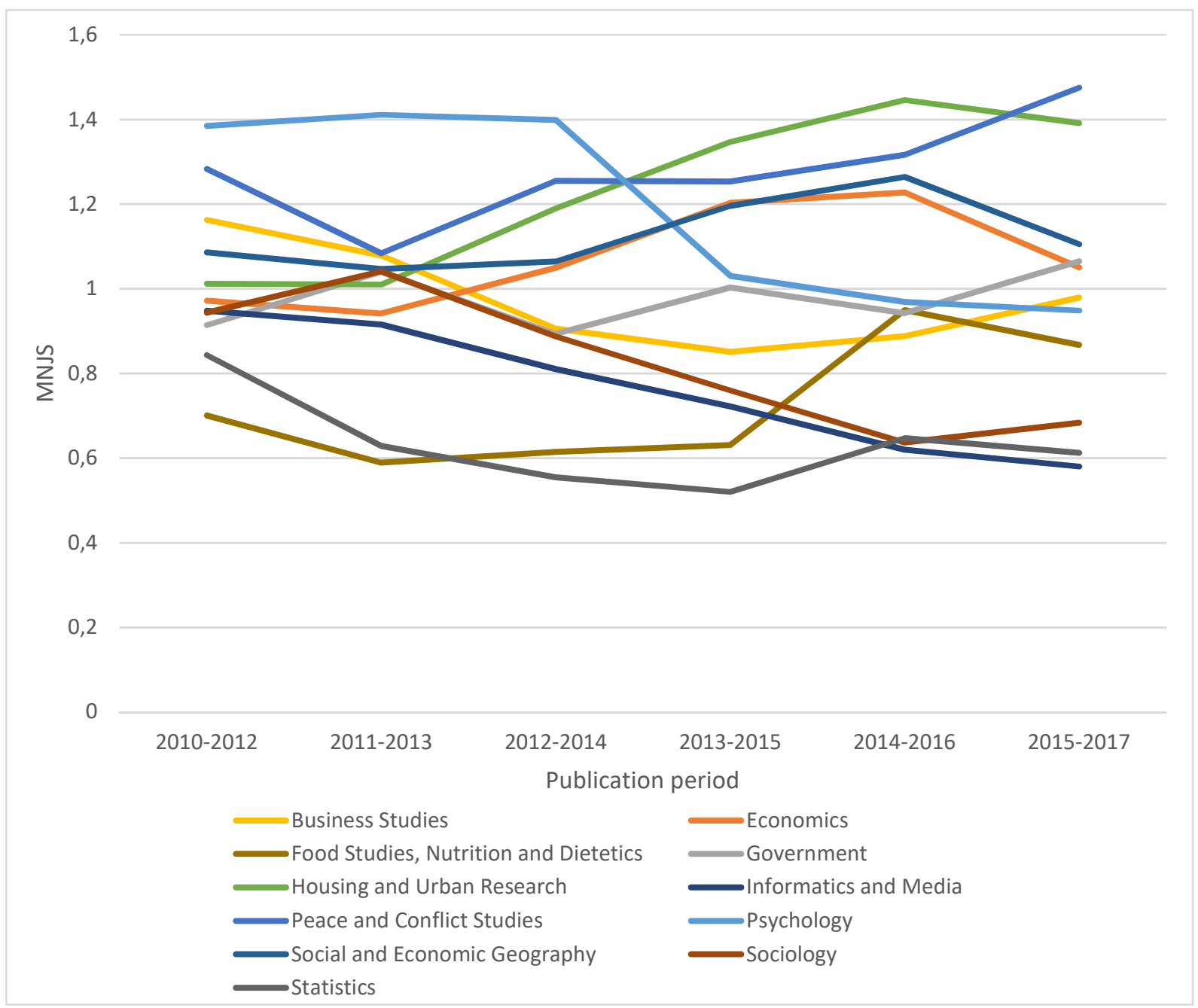

Figure 18. Faculty of Social Sciences. MNJS by publication period. 3-year moving average. 


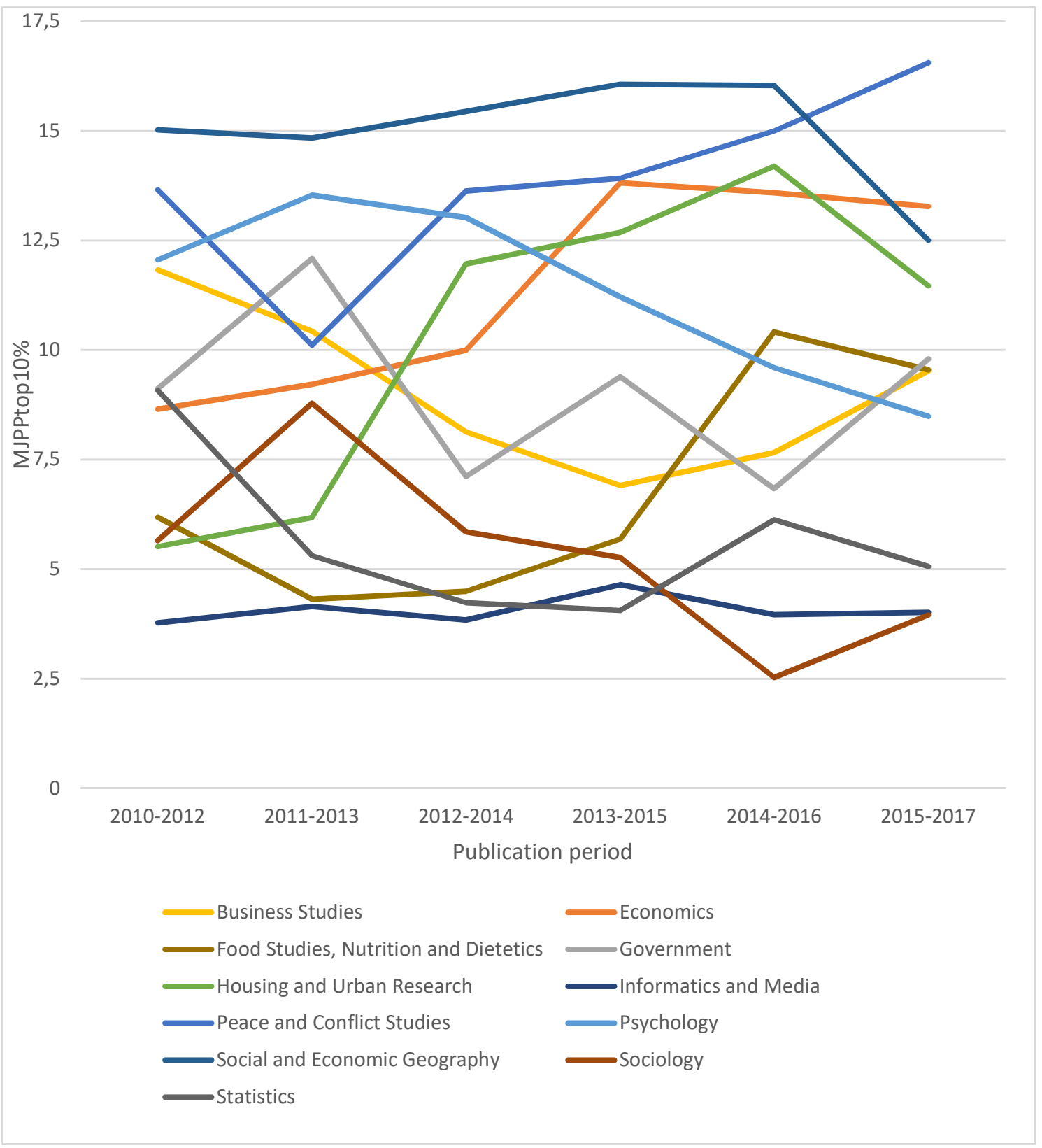

Figure 19. Faculty of Social Sciences. MJPPtop10\% by publication period. 3-year moving average.

\subsubsection{Collaboration}

For all tables in this section, PP(int collab) and PP(industry) are given as percentages.

\section{Faculty of Arts}

Table 15. Faculty of Arts. Publications (P, full counts), PP(int collab) and PP(industry) for the whole publication period 2010-2017

\begin{tabular}{lccc}
\hline Department & \multicolumn{3}{c}{ Indicator } \\
& P & PP(int collab) & PP(industry) \\
\hline ABM & 43 & 34,9 & 0,0 \\
Archeology and Ancient History & 70 & 68,6 & 1,4 \\
Art History & 9 & 88,9 & 0,0 \\
Cultural Anthropology and Ethnology & 11 & 18,2 & 0,0 \\
Game Design & 1 & 100,0 & 100,0 \\
Gender Research & 24 & 25,0 & 4,2 \\
History & 17 & 11,8 & 0,0
\end{tabular}




\begin{tabular}{lccc} 
History of Sciences and Ideas & 6 & 16,7 & 0,0 \\
Literature & 0 & - & - \\
Musicology & 2 & 100 & 0,0 \\
Philosophy & 27 & 11,1 & 0,0 \\
\hline
\end{tabular}

In Table 16, which gives values of PP(int collab) and PP(industry) by publication period, “_“ in a table cell indicates that indicator values for the corresponding department and period cannot be obtained, since the department has no WoS publications (of the type "Article" or "Review") in the period.

Table 16. Faculty of Arts. PP(int collab) and PP(industry) (within parentheses) by publication period. 3-year moving average.

\begin{tabular}{|c|c|c|c|c|c|c|}
\hline \multicolumn{7}{|l|}{ Department } \\
\hline & 2010-2012 & 2011-2013 & 2012-2014 & 2013-2015 & 2014-2016 & 2015-2017 \\
\hline $\mathrm{ABM}$ & $25,0(0,0)$ & $38,5(0,0)$ & $44,4(0,0)$ & $53,9(0,0)$ & $37,6(0,0)$ & $40,0(0,0)$ \\
\hline \multicolumn{7}{|l|}{ Archeology and } \\
\hline Ancient History & $61,1(5,6)$ & $50,0(5,6)$ & $55,0(5,0)$ & $66,7(0,0)$ & $75,8(0,0)$ & $77,5(0,0)$ \\
\hline Art History & - & - & $100,0(0,0)$ & $100,0(0,0)$ & $100,0(0,0)$ & $87,6(0,0)$ \\
\hline \multicolumn{7}{|l|}{ Cultural } \\
\hline \multicolumn{7}{|l|}{ Anthropology and } \\
\hline Ethnology & $33,3(0,0)$ & $0,0(0,0)$ & $0,0(0,0)$ & $20,0(0,0)$ & $16,7(0,0)$ & $16,7(0,0)$ \\
\hline Game Design & - & - & - & - & $100(100,0)$ & $100(100,0)$ \\
\hline Gender Research & $25,0(0,0)$ & $0,0(0,0)$ & $28,6(0,0)$ & $25,0(0,0)$ & $38,5(7,7)$ & $21,4(7,1)$ \\
\hline History & $0(0,0)$ & $0(0,0)$ & $20,0(0,0)$ & $20,0(0,0)$ & $25,0(0,0)$ & $0,0(0,0)$ \\
\hline \multicolumn{7}{|l|}{ History of Sciences } \\
\hline and Ideas & - & - & $0,0(0,0)$ & $0,0(0,0)$ & $0,0(0,0)$ & $20,0(0,0)$ \\
\hline Literature & - & - & - & - & - & - \\
\hline Musicology & $100(0,0)$ & $100(0,0)$ & - & $100(0,0)$ & $100(0,0)$ & $100(0,0)$ \\
\hline Philosophy & $13,3(0,0)$ & $8,3(0,0)$ & $10,0(0,0)$ & $0,0(0,0)$ & $0,0(0,0)$ & $16,7(0,0)$ \\
\hline
\end{tabular}

\section{Faculty of Languages}

Table 17. Faculty of Languages. Publications (P, full counts), PP(int collab) and PP(industry) for the whole publication period 2010-2017.

\begin{tabular}{lccc}
\hline Department & \multicolumn{2}{c}{ Indicator } \\
& P & PP(int collab) & PP(industry) \\
\hline English & 9 & 11,1 & 0,0 \\
Linguistics and Philology & 30 & 60,0 & 6,7 \\
Modern Languages & 14 & 0,0 & 0,0 \\
Scandinavian Languages & 12 & 16,7 & 0,0 \\
\hline
\end{tabular}

Table 18. Faculty of Languages. PP(int collab) and PP(industry) (within parentheses) by publication period. 3year moving average.

\begin{tabular}{lcccccc}
\hline Department & $\mathbf{2 0 1 0 - 2 0 1 2}$ & $\mathbf{2 0 1 1 - 2 0 1 3}$ & $\mathbf{2 0 1 2 - 2 0 1 4}$ & $\mathbf{2 0 1 3 - 2 0 1 5}$ & $\mathbf{2 0 1 4 - 2 0 1 6}$ & $\mathbf{2 0 1 5 - 2 0 1 7}$ \\
\hline English & $0,0(0,0)$ & $0,0(0,0)$ & $50,0(0,0)$ & $50,0(0,0)$ & $25,0(0,0)$ & $0,0(0,0)$ \\
Linguistics and & & & & & & \\
Philology & $60,0(0,0)$ & $50,0(7,1)$ & $50,0(14,3)$ & $50,0(12,5)$ & $66,7(11,1)$ & $69,2(0,0)$ \\
Modern Languages & $0,0(0,0)$ & $0,0(0,0)$ & $0,0(0,0)$ & $0,0(0,0)$ & $0,0(0,0)$ & $0,0(0,0)$ \\
Scandinavian & - & $50,0(0,0)$ & $50,0(0,0)$ & $33,3(0,0)$ & $11,1(0,0)$ & $0,0(0,0)$ \\
Languages & - & & & & & \\
\hline
\end{tabular}


Faculty of Education, Faculty of Law and Faculty of Theology

Table 19. Faculty of Education, Faculty of Law and Faculty of Theology. Publications (P, full counts), PP(int collab) and PP(industry) for the whole publication period 2010-2017.

\begin{tabular}{lccc}
\hline Department & \multicolumn{2}{c}{ Indicator } \\
& P & PP(int collab) & PP(industry) \\
\hline Faculty of Education & 97 & 21,7 & 0,0 \\
Faculty of Law & 20 & 35,0 & 0,0 \\
Faculty of Theology & 23 & 39,1 & 0,0 \\
\hline
\end{tabular}

Table 20. Faculty of Education, Faculty of Law and Faculty of Theology. PP(int collab) and PP(industry) (within parentheses) by publication period. 3-year moving average.

\begin{tabular}{lcccccc}
\hline Department & $\mathbf{2 0 1 0 - 2 0 1 2}$ & $\mathbf{2 0 1 1 - 2 0 1 3}$ & $\mathbf{2 0 1 2 - 2 0 1 4}$ & $\mathbf{2 0 1 3 - 2 0 1 5}$ & $\mathbf{2 0 1 4 - 2 0 1 6}$ & $\mathbf{2 0 1 5 - 2 0 1 7}$ \\
\hline Faculty of & & & & & & \\
Education & $12,0(0,0)$ & $8,0(0,0)$ & $14,8(0,0)$ & $18,2(0,0)$ & $30,6(0,0)$ & $27,3(0,0)$ \\
$\begin{array}{l}\text { Faculty of Law } \\
\text { Faculty of }\end{array}$ & $25,0(0,0)$ & $20,0(0,0)$ & $50,0(0,0)$ & $40,0(0,0)$ & $41,7(0,0)$ & $22,2(0,0)$ \\
Theology & & & & & & \\
\end{tabular}

\section{Faculty of Social Sciences}

Table 21. Faculty of Social Sciences. Publications (P, full counts), PP(int collab) and PP(industry) for the whole publication period 2010-2017.

\begin{tabular}{lccc}
\hline Department & \multicolumn{3}{c}{ Indicator } \\
& P & PP(int collab) & PP(industry) \\
\hline Business Studies & 146 & 54,8 & 0,7 \\
Russian and Eurasian Studies & 30 & 43,3 & 0,0 \\
Economic History & 29 & 37,9 & 0,0 \\
Economics & 184 & 41,8 & 2,2 \\
Food Studies, Nutrition and Dietetics & 69 & 46,4 & 1,5 \\
Government & 180 & 33,3 & 1,1 \\
Housing and Urban Research & 130 & 50,0 & 1,5 \\
Informatics and Media & 49 & 40,8 & 4,1 \\
Peace and Conflict Studies & 135 & 38,5 & 0,0 \\
Psychology & 478 & 42,3 & 2,5 \\
Social and Economic Geography & 110 & 29,1 & 0,0 \\
Sociology & 94 & 24,5 & 0,0 \\
Statistics & 79 & 38,0 & 0,0 \\
\hline
\end{tabular}

Table 22. Faculty of Social Sciences. PP(int collab) and PP(industry) (within parentheses) publication period. 3year moving average.

\begin{tabular}{lcccccc}
\hline Department & $\mathbf{2 0 1 0 - 2 0 1 2}$ & $\mathbf{2 0 1 1 - 2 0 1 3}$ & $\mathbf{2 0 1 2 - 2 0 1 4}$ & $\mathbf{2 0 1 3 - 2 0 1 5}$ & $\mathbf{2 0 1 4 - 2 0 1 6}$ & $\mathbf{2 0 1 5 - 2 0 1 7}$ \\
\hline $\begin{array}{l}\text { Business Studies } \\
\text { Russian and }\end{array}$ & $47,2(1,9)$ & $45,6(1,8)$ & $51,7(0,0)$ & $57,4(0,0)$ & $63,1(0,0)$ & $64,2(0,0)$ \\
Eurasian Studies & $50,0(0,0)$ & $66,7(0,0)$ & $40,0(0,0)$ & $25,0(0,0)$ & $30,8(0,0)$ & $45,5(0,0)$ \\
Economic History & $33,3(0,0)$ & $40,0(0,0)$ & $42,9(0,0)$ & $42,9(0,0)$ & $42,9(0,0)$ & $30,0(0,0)$ \\
Economics & $41,6(6,2)$ & $40,9(5,6)$ & $44,9(5,1)$ & $46,2(0,0)$ & $47,0(0,0)$ & $40,3(0,0)$ \\
Food Studies, & & & & & & \\
Nutrition and & $22,2(0,0)$ & $27,3(0,0)$ & $21,1(0,0)$ & $40,9(0,0)$ & $56,0(0,0)$ & $67,6(2,7)$ \\
Dietetics & $26,7(0,0)$ & $27,4(0,0)$ & $31,0(1,7)$ & $29,8(1,5)$ & $35,8(2,5)$ & $36,4(1,1)$ \\
Government & & & & & \\
Housing and Urban & & & & & & \\
Research & $55,0(2,5)$ & $47,4(5,3)$ & $41,2(3,9)$ & $43,9(1,8)$ & $50,0(0,0)$ & $51,9(0,0)$
\end{tabular}


Informatics and

Media

$26,3(0,0) \quad 23,8(0,0)$

$26,9(7,7)$

$41,7(8,3)$

$63,1(10,5)$

$83,3(0,0)$

Peace and Conflict

Studies

Psychology

$33,4(0,0) \quad 38,5(0,0)$

$36,1(0,0)$

$41,1(0,0)$

$39,0(0,0)$

$38,5(0,0)$

Social and

$42,6(3,1)$

$38,8(2,3)$

$43,1(2,9)$

$42,3(2,8)$

$48,4(3,2)$

$42,9(2,5)$

Economic

Geography

$24,3(0,0)$

$24,3(0,0)$

$20,0(0,0)$

$30,5(0,0)$

$37,5(0,0)$

$37,2(0,0)$

Sociology

$26,1(0,0)$

$18,5(0,0) \quad 21,6(0,0)$

$19,5(0,0)$

$17,1(0,0)$

$26,6(0,0)$

Statistics

$50,0(0,0)$

$40,0(0,0)$

$34,6(0,0)$

$25,0(0,0)$

$44,1(0,0)$

$40,4(0,0)$

\subsubsection{Open access}

For all figures in this section, proportion OA is given as percentages. OA is defined as publications belonging to any class of OA (Gold, green or hybrid). All OA measures use full counts.

\section{Faculty of Arts}

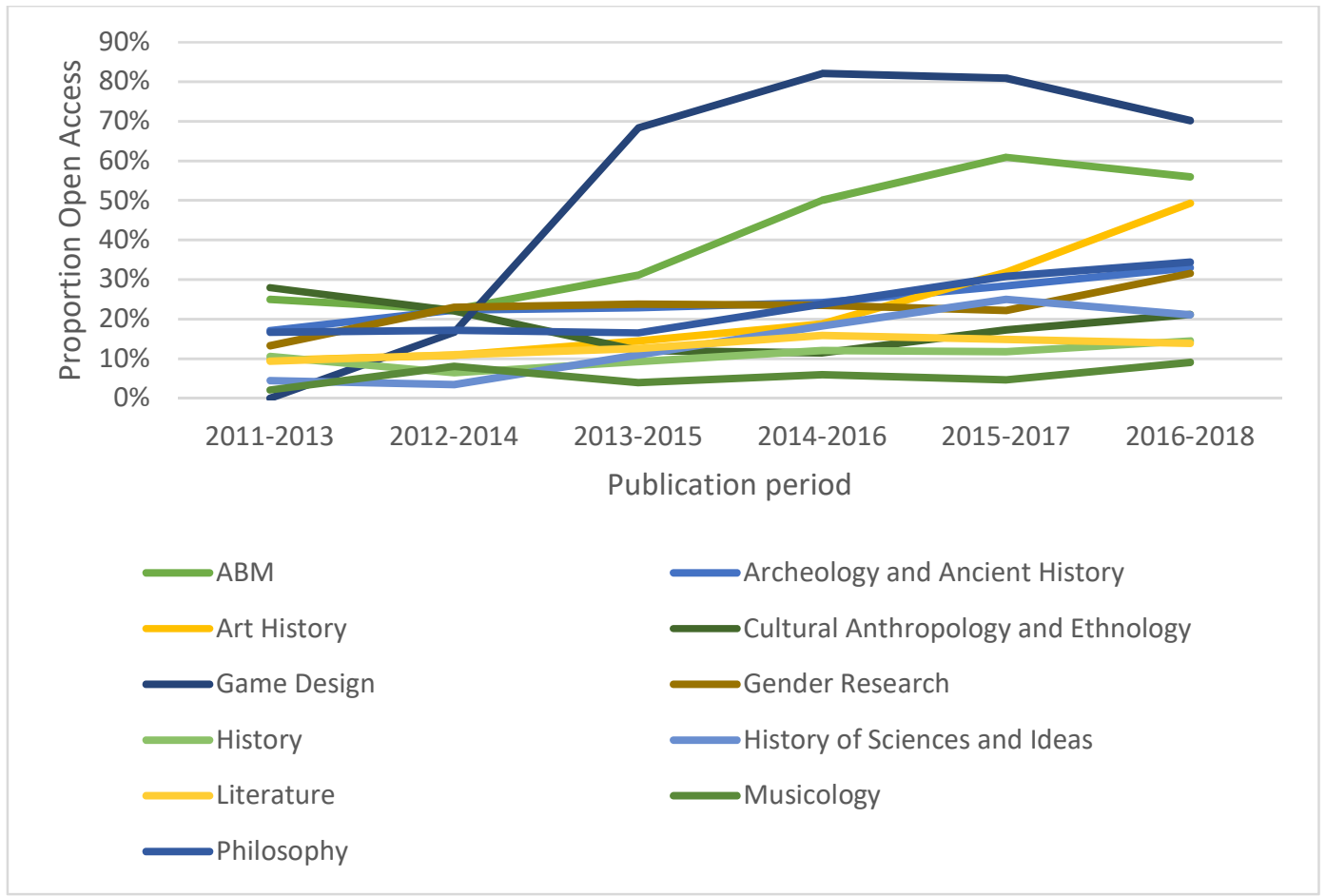

Figure 20. Faculty of Arts. Total Open Access by publication period. 3-year moving average. 
Faculty of Languages

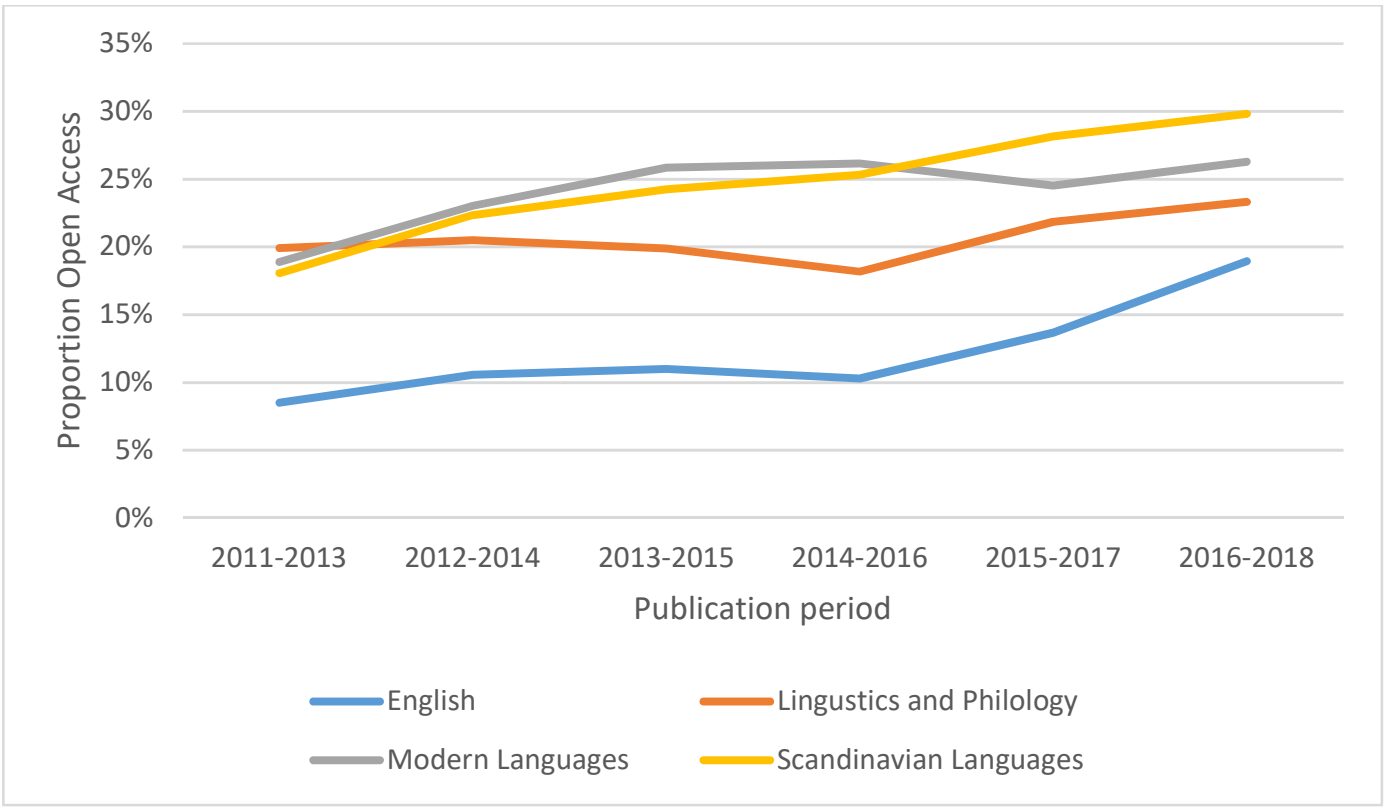

Figure 21. Faculty of Languages. Total Open Access by publication period. 3-year moving average.

Faculty of Education, Faculty of Law and Faculty of Theology

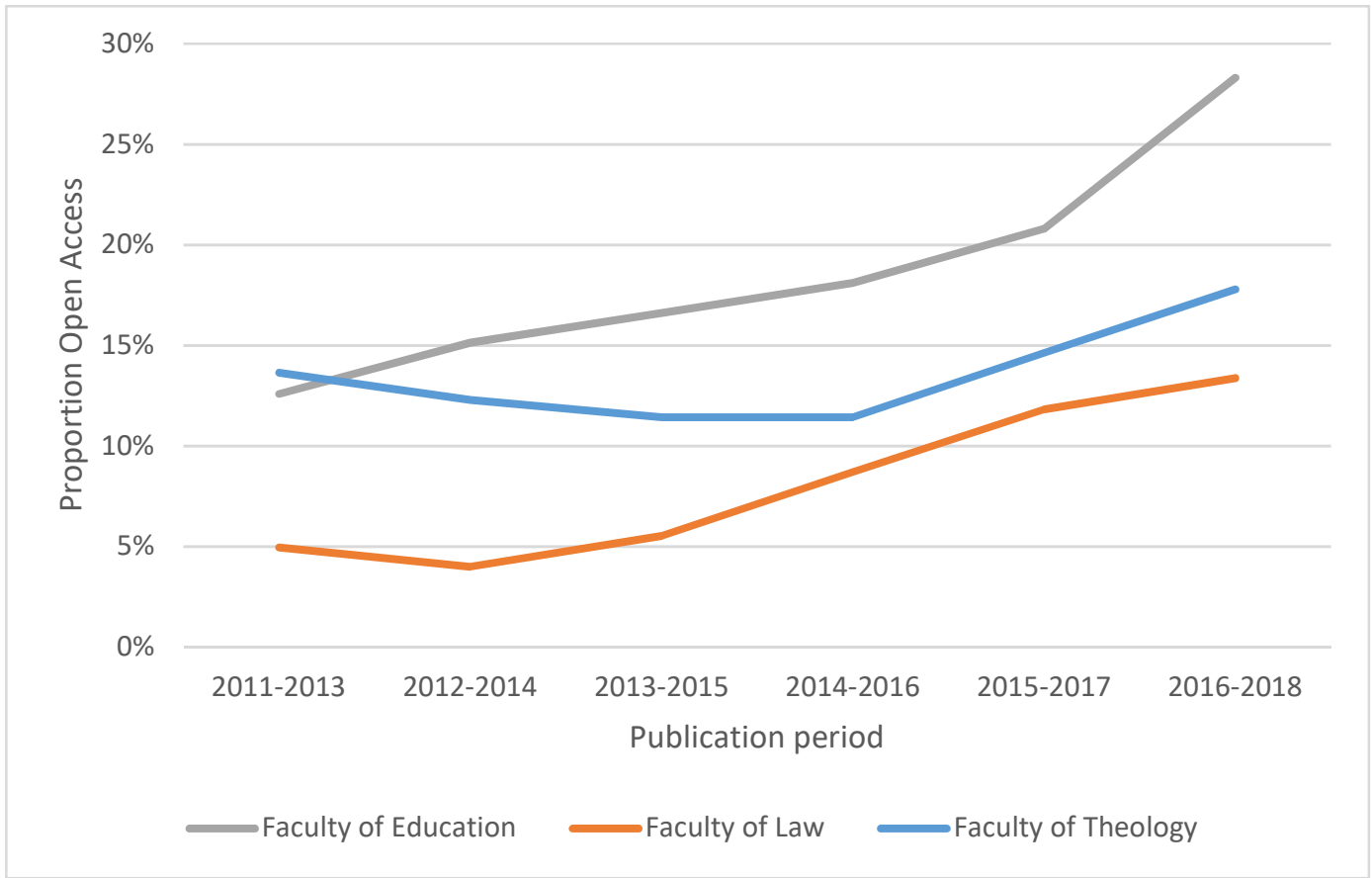

Figure 22. Faculty of Education, Faculty of Law and Faculty of Theology. Total Open Access by publication period. 3-year moving average. 


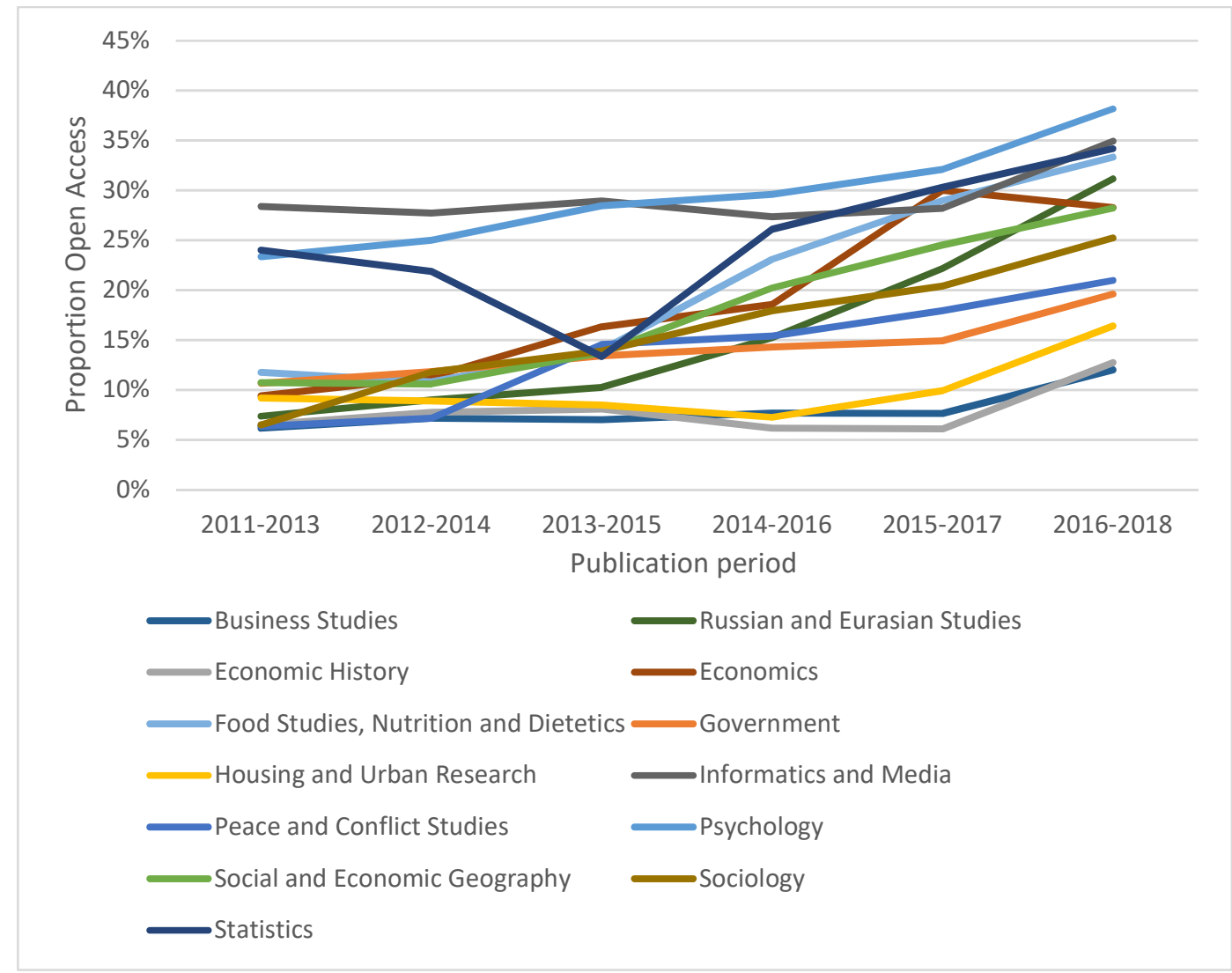

Figure 23. Faculty of Social Sciences. Total Open Access by publication period. 3-year moving average.

\subsection{MedFarm}

The number of MedFarm departments included in ABM is 11. Tables 23-24 report publication volume in terms of both fractional counts and full counts (within parentheses) by department and publication type, and WoS coverage. WoS coverage concerns the number of WoS publications of the four types represented in the tables relative to all publications of these types for the department.

Table 23. Faculty of Pharmacy. Publication volume by publication type, and WoS coverage (in \%). Publication period: 2010-2018. Data source: GLIS.

\begin{tabular}{lcccccc}
\hline Department & Article & $\begin{array}{c}\text { Article in } \\
\text { anthology }\end{array}$ & Monograph & $\begin{array}{c}\text { Ponfication type } \\
\text { Coper }\end{array}$ & Total & $\begin{array}{c}\text { WoS } \\
\text { coverage }\end{array}$ \\
\hline $\begin{array}{l}\text { Medicinal } \\
\text { Chemistry }\end{array}$ & $276,9(663)$ & $15,4(18)$ & $0,0(0)$ & $2,9(5)$ & $295,2(686)$ & 90,2 \\
$\begin{array}{l}\text { Pharmaceutical } \\
\text { Biosciences }\end{array}$ & $441,4(999)$ & $22,4(24)$ & $0,9(1)$ & $5,5(12)$ & $470,2(1036)$ & 88,4 \\
Pharmacy & $220,2(469)$ & $4,8(6)$ & $0,0(0)$ & $0,0(0)$ & $225,0(475)$ & 91,3 \\
\hline
\end{tabular}


Table 24. Faculty of Medicine. Publication volume by publication type, and WoS coverage (in \%). Publication period: 2010-2018. Data source: GLIS.

\begin{tabular}{|c|c|c|c|c|c|c|}
\hline \multirow[t]{2}{*}{ Department } & \multicolumn{6}{|c|}{ Publication type } \\
\hline & Article & $\begin{array}{l}\text { Article in } \\
\text { anthology }\end{array}$ & Monograph & $\begin{array}{c}\text { Conference } \\
\text { paper }\end{array}$ & Total & $\begin{array}{c}\text { WoS } \\
\text { coverage }\end{array}$ \\
\hline \multicolumn{7}{|l|}{ Immunology, } \\
\hline \multicolumn{7}{|l|}{ Genetics and } \\
\hline Pathology & $671,9(2500)$ & $20,2(31)$ & $0,3(1)$ & $5,6(19)$ & $698,0(2551)$ & 90,6 \\
\hline \multicolumn{7}{|l|}{ Medical } \\
\hline \multicolumn{7}{|l|}{ Biochemistry and } \\
\hline Microbiology & $382,2(1162)$ & 9,8 (19) & $0,3(1)$ & $1,1(4)$ & $393,4(1186)$ & 92,8 \\
\hline \multicolumn{7}{|l|}{ Medical Cell } \\
\hline Biology & $245,5(510)$ & $7,3(11)$ & $0,0(0)$ & $3,5(4)$ & $256,3(525)$ & 92,2 \\
\hline Medical Sciences & $1395,0(4974)$ & $33,5(44)$ & $2,9(3)$ & $7,2(16)$ & $1438,6(5037)$ & 86,3 \\
\hline Neuroscience & $965,7(2116)$ & $31,6(43)$ & $6,5(11)$ & $11,8(31)$ & $1015,7(2201)$ & 87,6 \\
\hline \multicolumn{7}{|l|}{ Public Health and } \\
\hline Caring Sciences & $850,1(2284)$ & $77,9(108)$ & $12,1(17)$ & $16,4(35)$ & $956,5(2444)$ & 72,5 \\
\hline Surgical Sciences & $1212,3(3309)$ & $67,0(89)$ & $3,1(4)$ & $18,5(38)$ & $1330,9(3440)$ & 78,8 \\
\hline \multicolumn{7}{|l|}{ Women's and } \\
\hline Children's Health & $853,0(2139)$ & $48,8(58)$ & $5,8(8)$ & $1,2(5)$ & $908,8(2210)$ & 84,4 \\
\hline
\end{tabular}

3.3.1 Publishing volume and publishing level-The Norwegian model

Faculty of Pharmacy

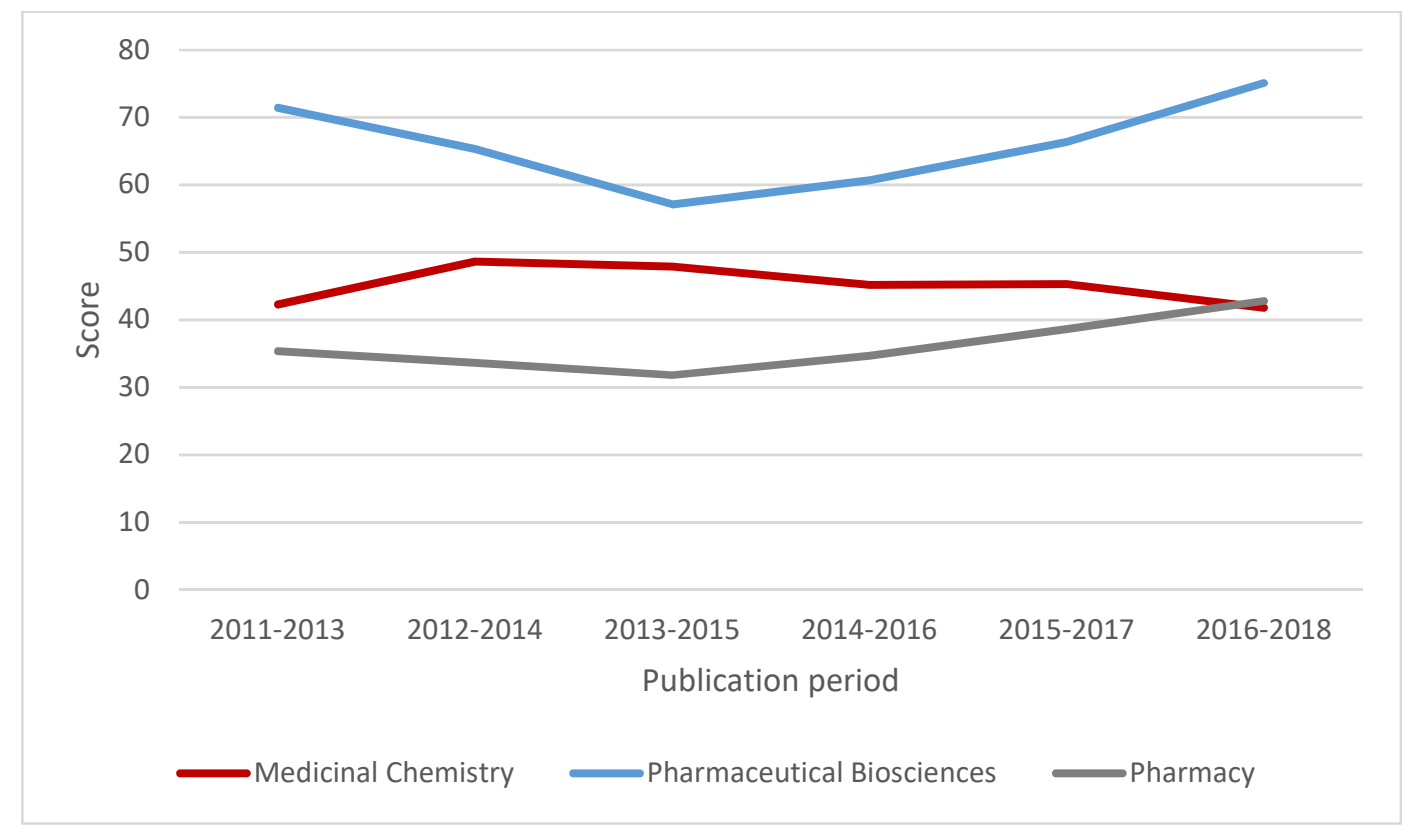

Figure 24. Faculty of Pharmacy. Norwegian score by publication period. 3-year moving average. 
Table 25. Faculty of Pharmacy. Proportion (in \%) publication fractions at level 2 relative to the sum of publication fractions across levels 1 and 2 (given within parentheses) by publication period. 3-year moving average.

\begin{tabular}{lcccccc}
\hline Department & $\mathbf{2 0 1 1 - 2 0 1 3}$ & $\mathbf{2 0 1 2 - 2 0 1 4}$ & $\mathbf{2 0 1 3 - 2 0 1 5}$ & $\mathbf{2 0 1 4 - 2 0 1 6}$ & $\mathbf{2 0 1 5 - 2 0 1 7}$ & $\mathbf{2 0 1 6 - 2 0 1 8}$ \\
\hline $\begin{array}{l}\text { Medicinal } \\
\text { Chemistry }\end{array}$ & $29,4(80,9)$ & $24,1(99,4)$ & $18,2(106,0)$ & $16,3(103,3)$ & $18,8(99,5)$ & $19,4(90,9)$ \\
$\begin{array}{l}\text { Pharmaceutical } \\
\text { Biosciences }\end{array}$ & $19,5(156,8)$ & $16,8(149,1)$ & $16,2(131,4)$ & $19,0(133,6)$ & $20,6(141,6)$ & $22,2(156,2)$ \\
Pharmacy & $28,6(67,9)$ & $29,8(64,8)$ & $26,9(63,7)$ & $28,3(68,0)$ & $28,1(74,4)$ & $31,9(78,6)$ \\
\hline
\end{tabular}

\section{Faculty of Medicine}

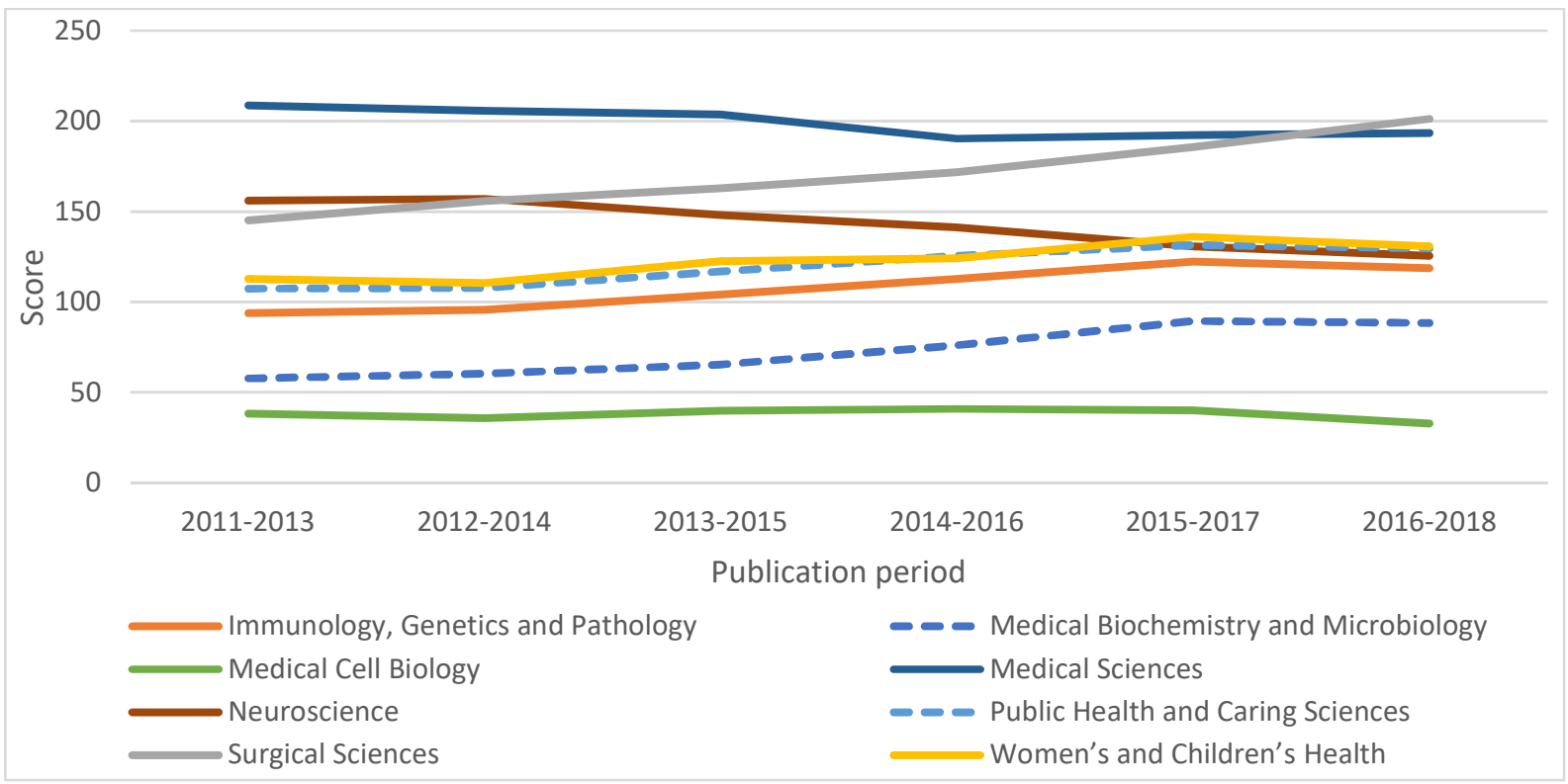

Figure 25. Faculty of Medicine. Norwegian score by publication period. 3-year moving average.

Table 26. Faculty of Medicine. Proportion (in \%) publication fractions at level 2 relative to the sum of publication fractions across levels 1 and 2 (given within parentheses) by publication period. 3-year moving average.

\begin{tabular}{lcccccc}
\hline Department & $\mathbf{2 0 1 1 - 2 0 1 3}$ & $\mathbf{2 0 1 2 - 2 0 1 4}$ & $\mathbf{2 0 1 3 - 2 0 1 5}$ & $\mathbf{2 0 1 4 - 2 0 1 6}$ & $\mathbf{2 0 1 5 - 2 0 1 7}$ & $\mathbf{2 0 1 6 - 2 0 1 8}$ \\
\hline $\begin{array}{l}\text { Immunology, } \\
\begin{array}{l}\text { Genetics and } \\
\text { Pathology }\end{array}\end{array}$ & $20,1(201,4)$ & $20,8(203,6)$ & $21,4(220,7)$ & $22,2(236,0)$ & $23,5(251,7)$ & $24,5(239,7)$ \\
$\begin{array}{l}\text { Medical } \\
\text { Biochemistry }\end{array}$ & & & & & & \\
$\begin{array}{l}\text { and } \\
\text { Microbiology }\end{array}$ & $35,0(102,5)$ & $31,2(111,5)$ & $32,4(118,6)$ & $32,0(139,0)$ & $34,5(159,3)$ & $34,5(157,5)$ \\
$\begin{array}{l}\text { Medical Cell } \\
\text { Biology }\end{array}$ & $19,4(83,4)$ & $18,3(79,0)$ & $19,8(85,8)$ & $19,3(88,5)$ & $18,6(87,6)$ & $19,0(71,3)$ \\
$\begin{array}{l}\text { Medical } \\
\text { Sciences }\end{array}$ & $19,0(455,6)$ & $19,7(446,6)$ & $18,8(448,6)$ & $17,2(427,2)$ & $16,3(437,9)$ & $16,8(437,3)$ \\
$\begin{array}{l}\text { Neuroscience } \\
\text { Public Health } \\
\text { and Caring }\end{array}$ & $18,5(339,0)$ & $16,6(350,3)$ & $15,7(339,2)$ & $15,5(324,5)$ & $14,2(307,3)$ & $12,8(298,6)$ \\
$\begin{array}{l}\text { Sciences } \\
\text { Surgical }\end{array}$ & $14,9(249,1)$ & $13,5(257,1)$ & $13,7(278,3)$ & $15,9(290,6)$ & $16,6(302,9)$ & $17,5(295,6)$ \\
Sciences & $15,5(335,4)$ & $17,1(348,6)$ & $17,9(359,2)$ & $16,5(387,1)$ & $15,2(430,7)$ & $15,6(464,4)$ \\
\hline
\end{tabular}




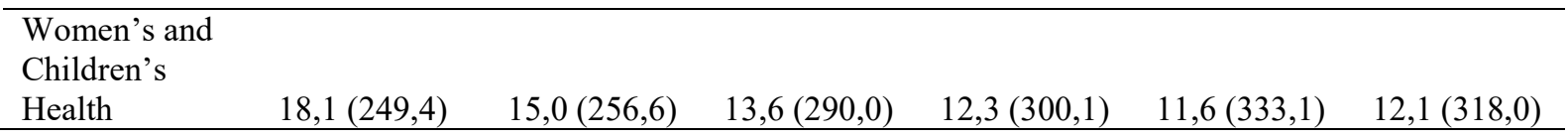

\subsubsection{Field normalized citation impact}

\section{Faculty of Pharmacy}

Table 27. Faculty of Pharmacy. Publication fractions (P; full counts within parentheses), MNCS, PP(top 10\%), MNJS and MJPPtop10\% for the whole publication period 2010-2017.

\begin{tabular}{|c|c|c|c|c|c|}
\hline \multirow[t]{2}{*}{ Department } & \multicolumn{5}{|c|}{ Indicator } \\
\hline & $\mathbf{P}$ & MNCS & PP(top 10\%) & MNJS & МJPPtop10\% \\
\hline Medicinal Chemistry & $215,6(526)$ & 0,89 & 8,2 & 0,90 & 8,1 \\
\hline Pharmaceutical Biosciences & $331,4(781)$ & 1,02 & 8,3 & 1,02 & 9,4 \\
\hline Pharmacy & $166,9(367)$ & 1,20 & 19,0 & 1,34 & 17,5 \\
\hline
\end{tabular}

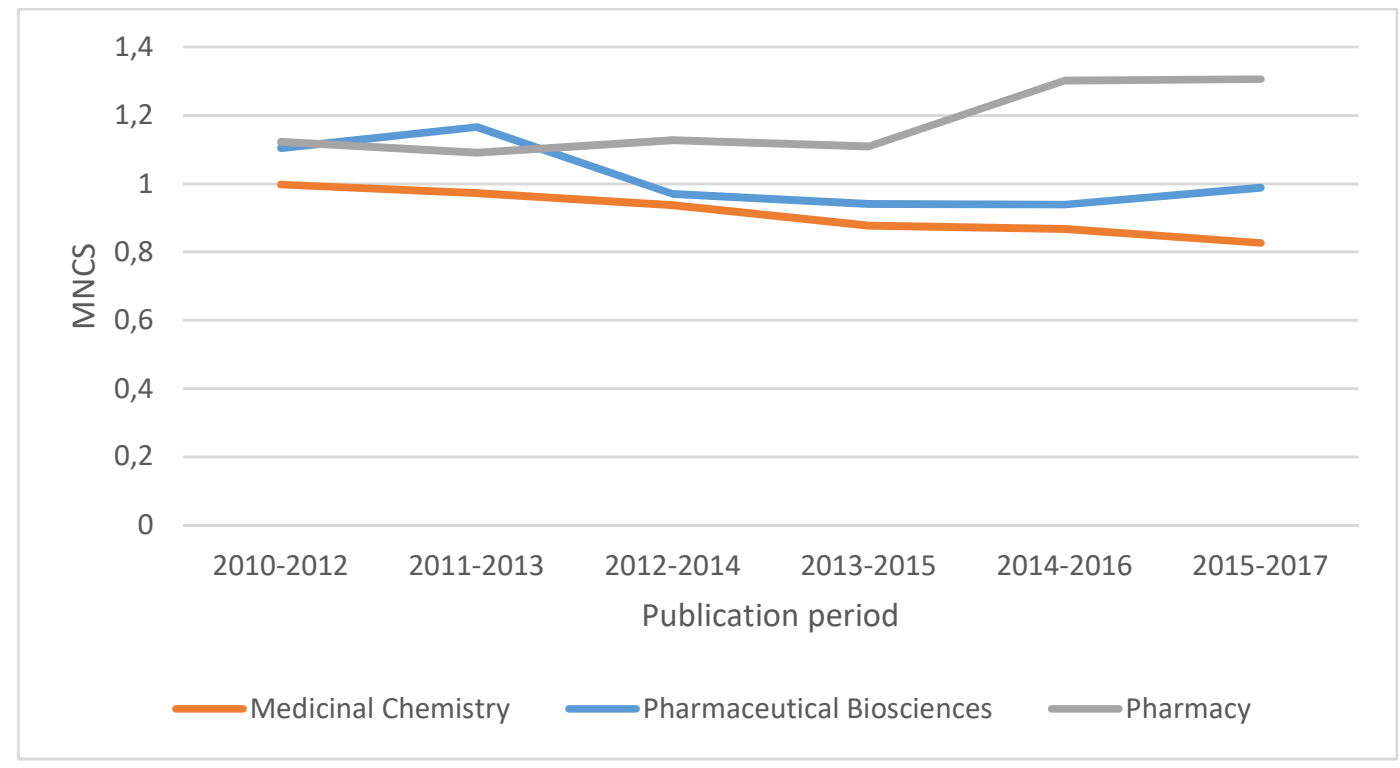

Figure 26. Faculty of Pharmacy. MNCS by publication period. 3-year moving average. 


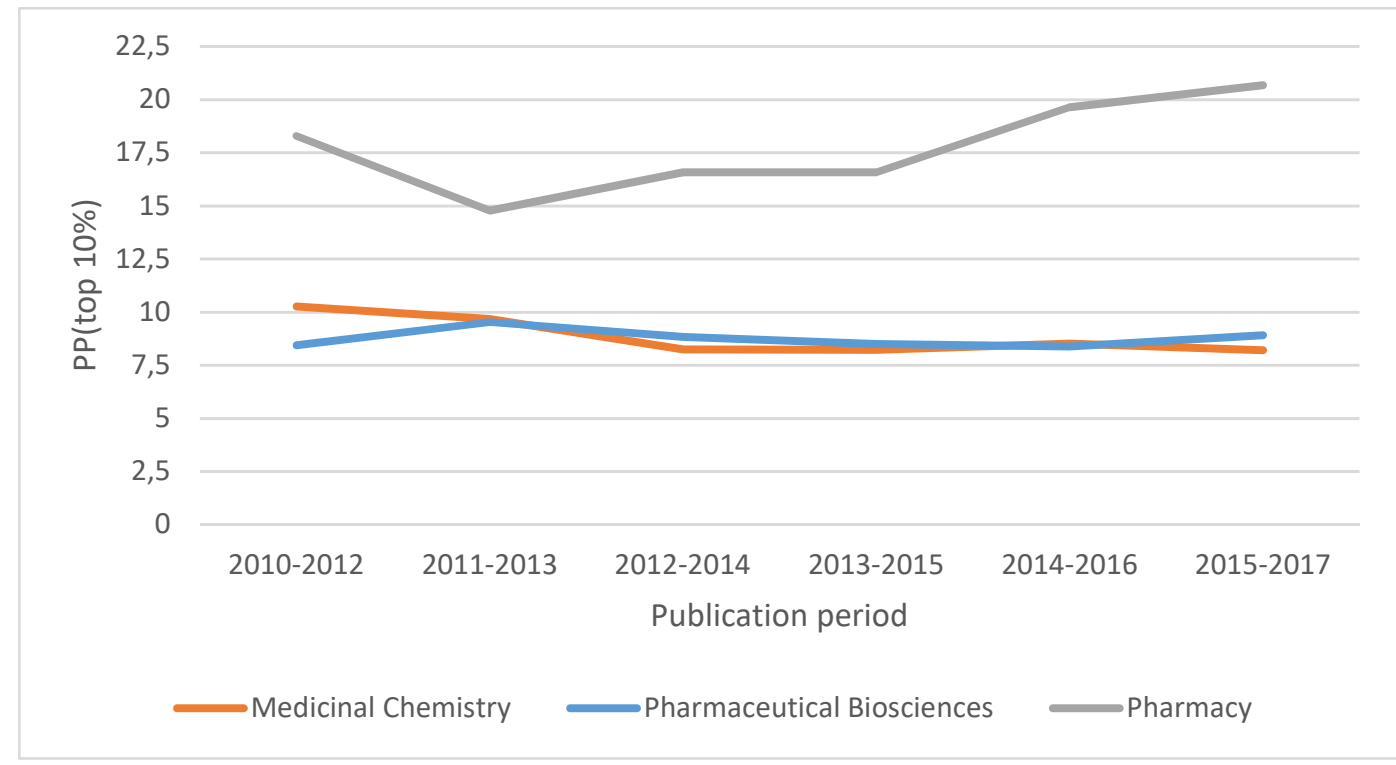

Figure 27. Faculty of Pharmacy. PP(top 10\%) by publication period. 3-year moving average.

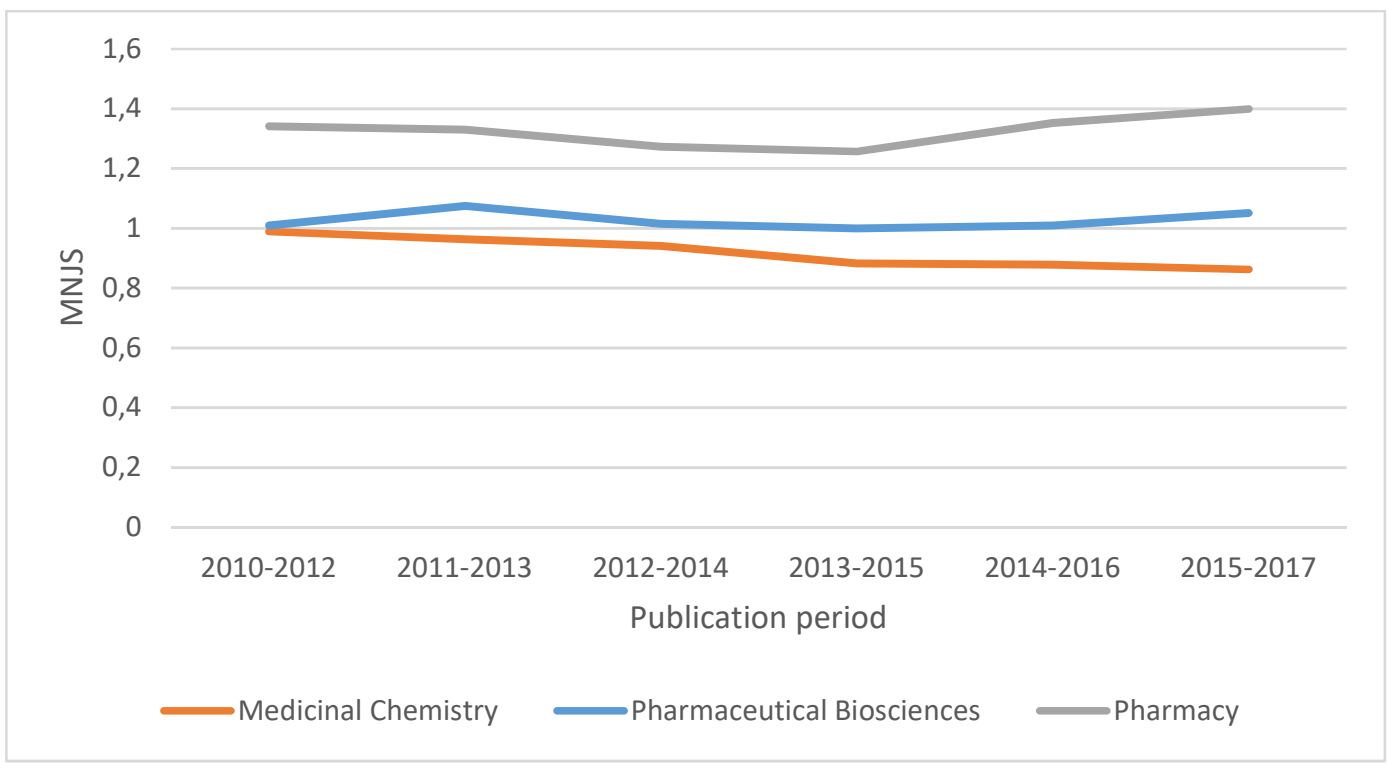

Figure 28. Faculty of Pharmacy. MNJS by publication period. 3-year moving average. 


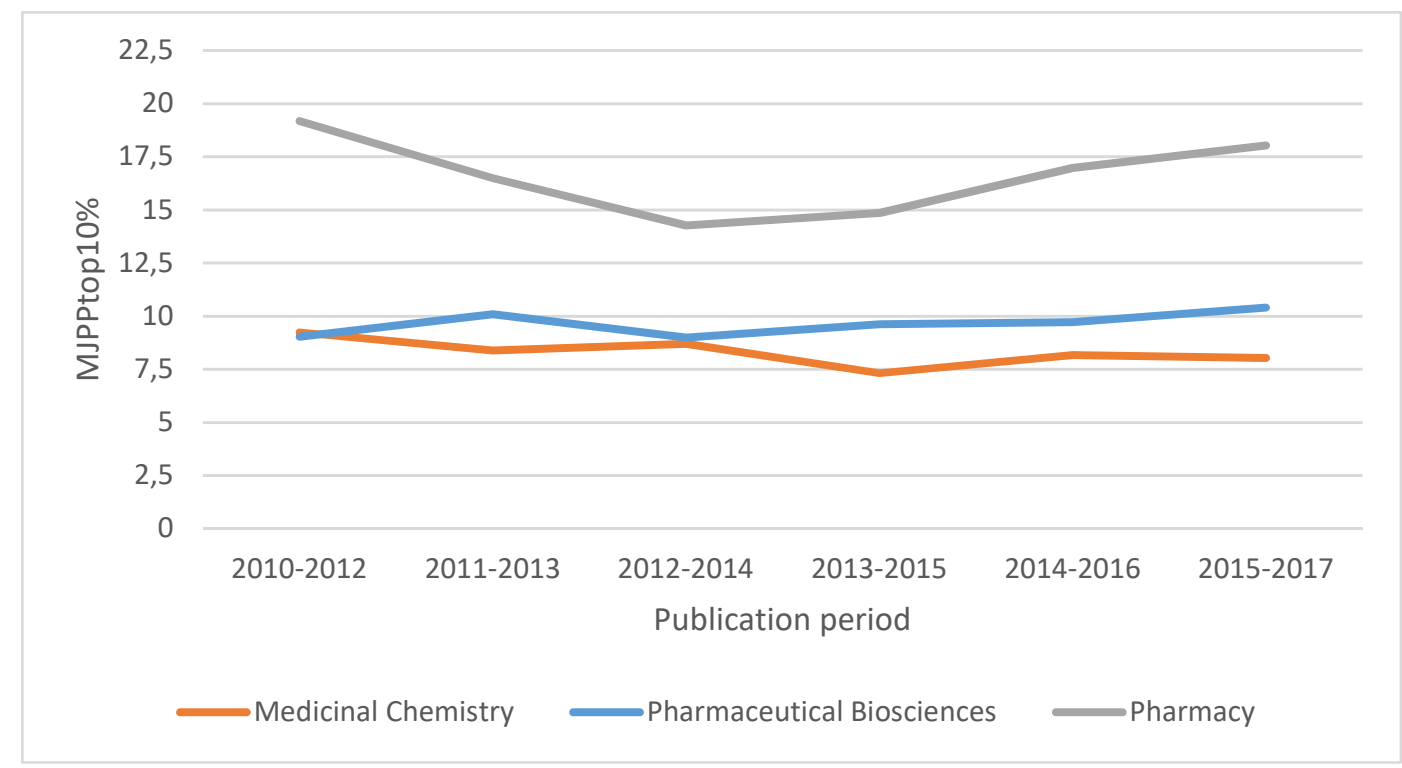

Figure 29. Faculty of Pharmacy. MJPPtop10\% by publication period. 3-year moving average.

\section{Faculty of Medicine}

Table 28. Faculty of Medicine. Publication fractions (P; full counts within parentheses), MNCS, PP(top 10\%), MNJS and MJPPtop10\% for the whole publication period 2010-2017.

\begin{tabular}{lccccc}
\hline Department & P & MNCS & $\begin{array}{c}\text { Indicator } \\
\text { PP(top } \\
\mathbf{1 0 \%})\end{array}$ & MNJS & MJPPtop10\% \\
\hline Immunology, Genetics and Pathology & $796,1(2624)$ & 1,33 & 13,9 & 1,31 & 14,1 \\
Medical Biochemistry and & & & & & \\
Microbiology & $320,6(924)$ & 1,48 & 15,7 & 1,54 & 16,1 \\
Medical Cell Biology & $195,9(421)$ & 1,03 & 11,6 & 0,99 & 10,1 \\
Medical Sciences & $1027,1(3892)$ & 1,16 & 12,4 & 1,14 & 12,1 \\
Neuroscience & $773,2(1853)$ & 0,96 & 9,2 & 1,00 & 9,6 \\
Public Health and Caring Sciences & $492,4(1639)$ & 0,97 & 9,2 & 0,99 & 9,9 \\
Surgical Sciences & $816,7(2398)$ & 1,09 & 11,1 & 1,10 & 11,5 \\
Women's and Children's Health & $588,7(1670)$ & 0,91 & 8,4 & 0,94 & 8,9 \\
\hline
\end{tabular}




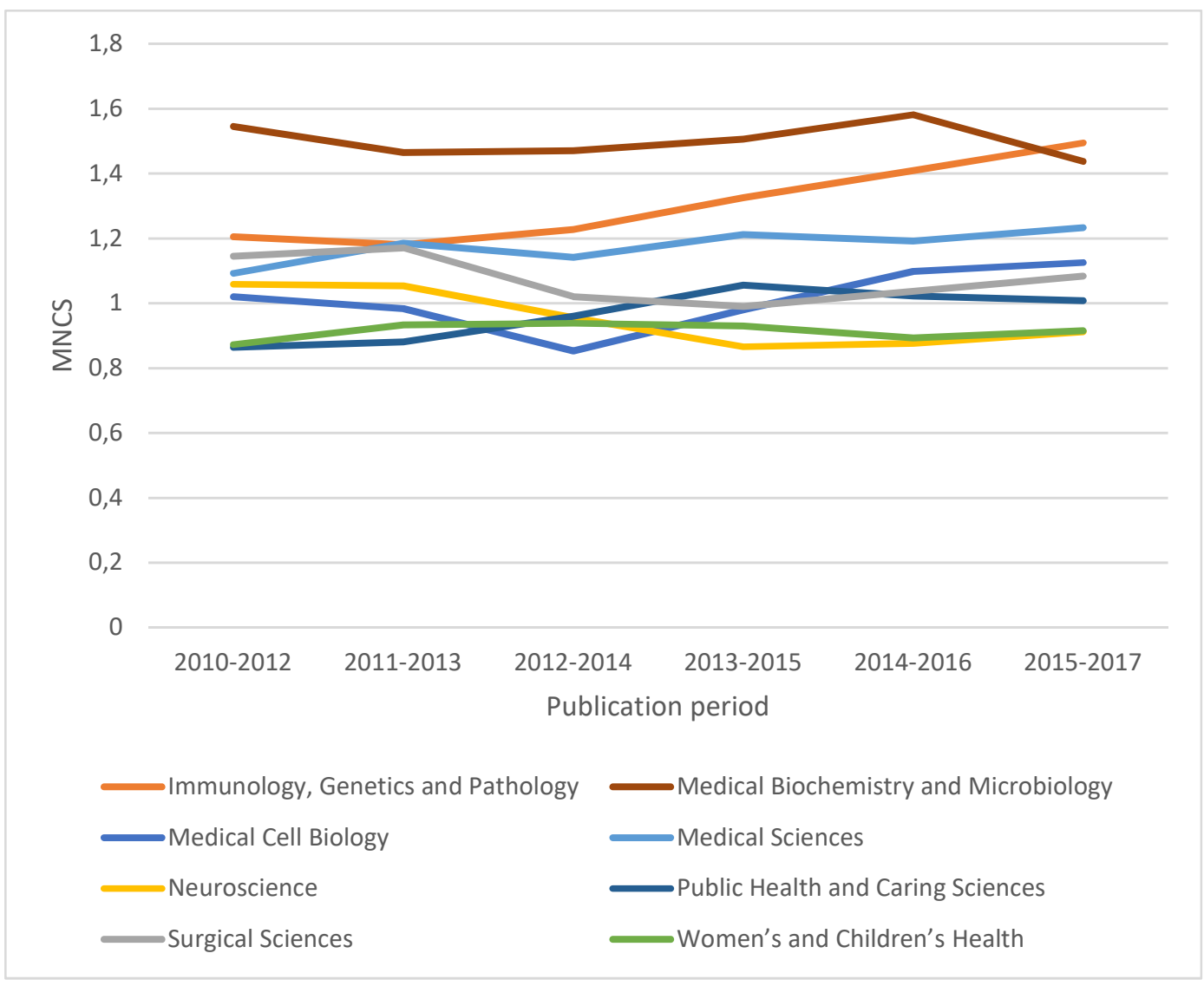

Figure 30. Faculty of Medicine. MNCS by publication period. 3-year moving average.

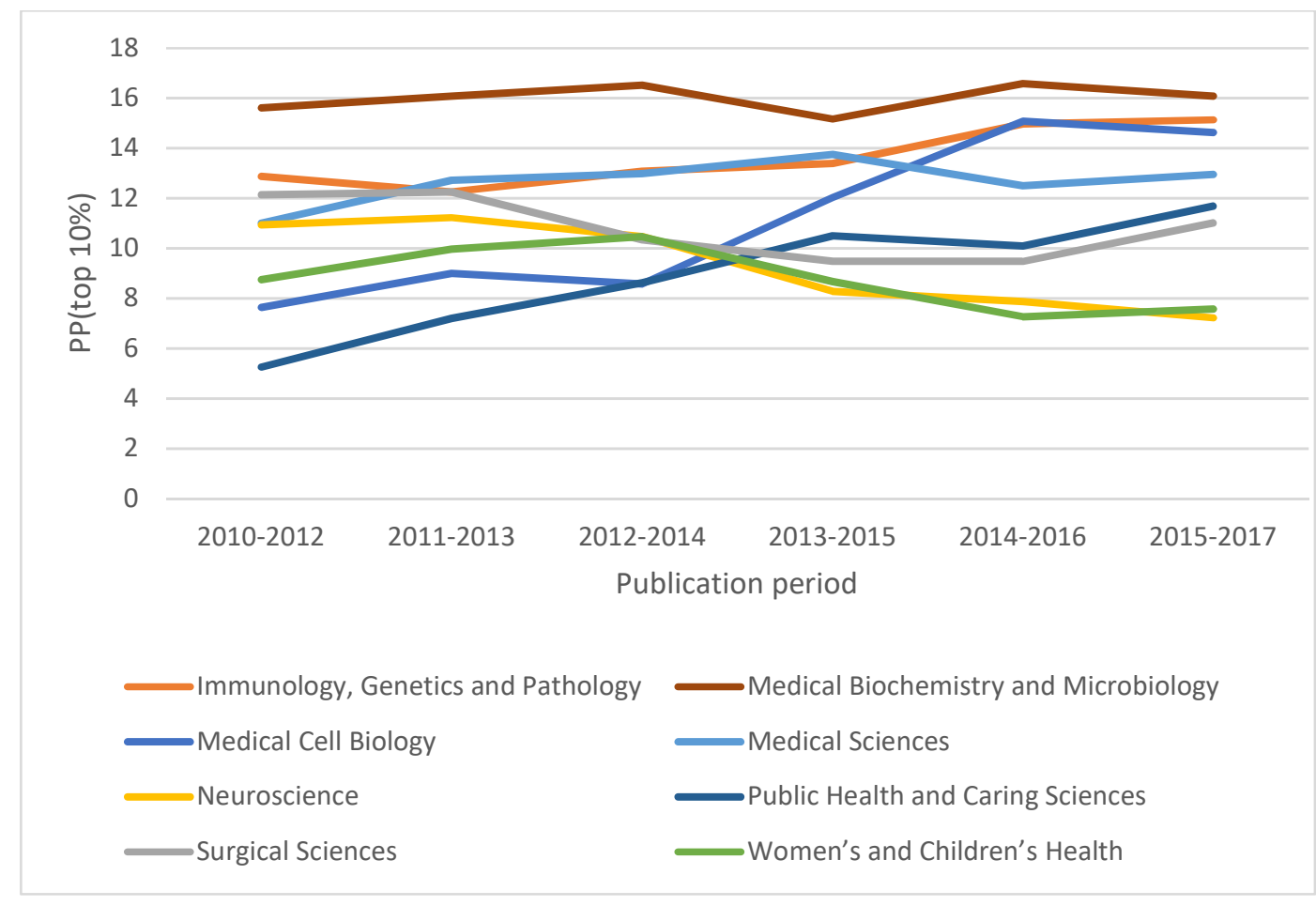

Figure 31. Faculty of Medicine. PP(top 10\%) by publication period. 3-year moving average. 


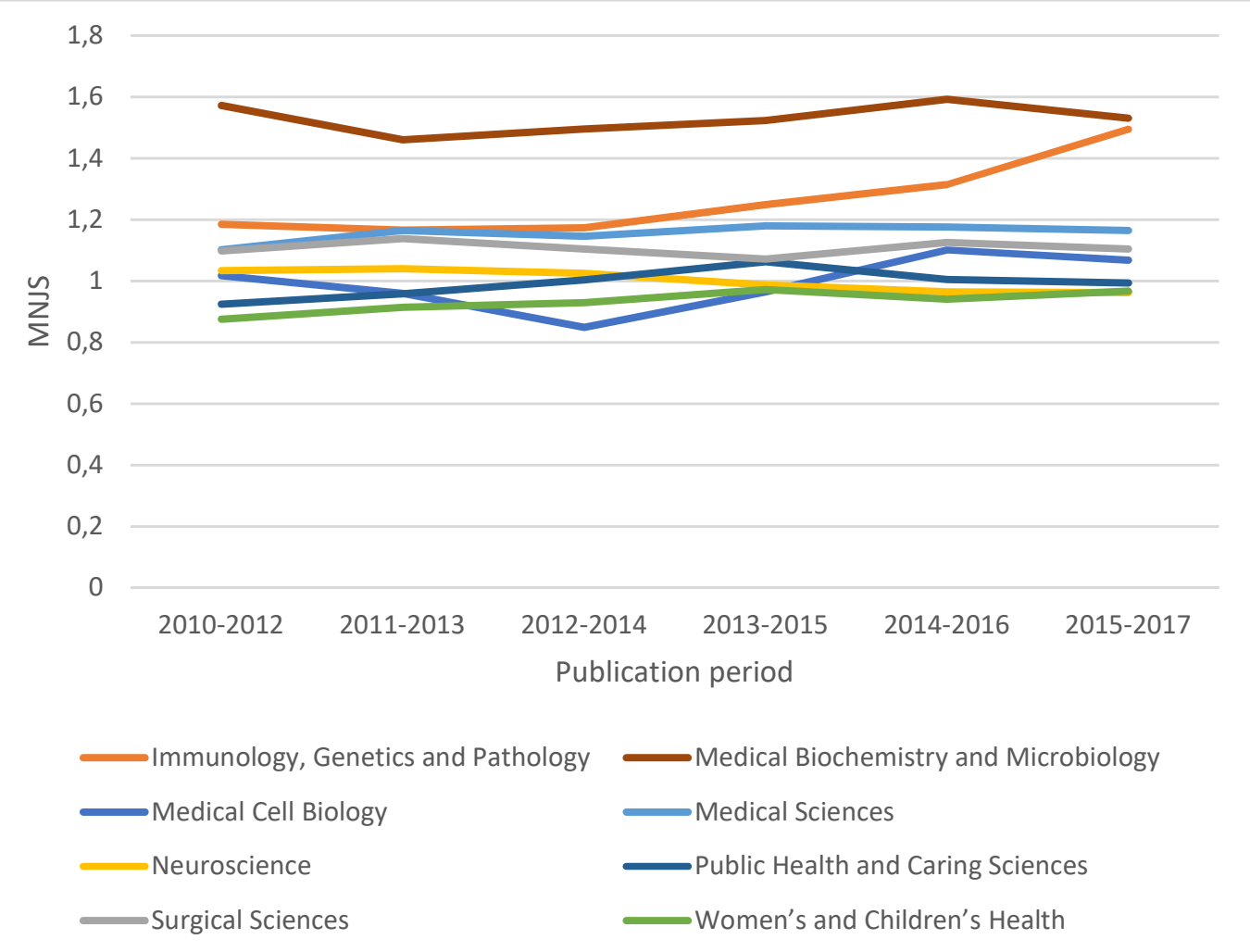

Figure 32. Faculty of Medicine. MNJS by publication period. 3-year moving average.

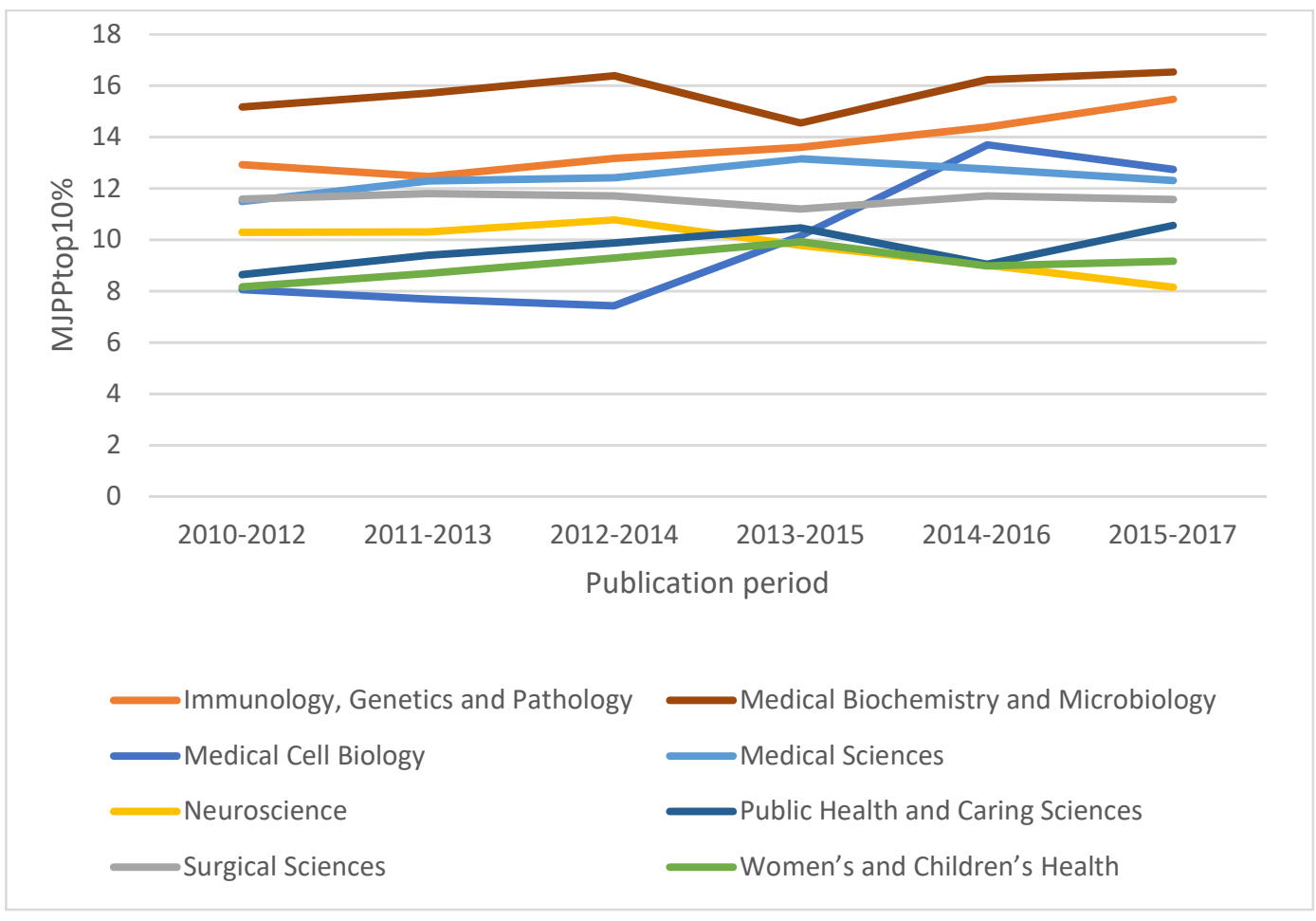

Figure 33. Faculty of Medicine. MJPPtop10\% by publication period. 3-year moving average.

\subsubsection{Collaboration}

For all tables in this section, $\mathrm{PP}($ int collab) and $\mathrm{PP}($ industry) are given as percentages. 
Faculty of Pharmacy

Table 29. Faculty of Pharmacy. Publications (P, full counts), PP(int collab) and PP(industry) for the whole publication period 2010-2017.

\begin{tabular}{lccc}
\hline Department & \multicolumn{3}{c}{ Indicator } \\
& P & PP(int collab) & PP(industry) \\
\hline Medicinal Chemistry & 526 & 46,0 & 20,2 \\
Pharmaceutical Biosciences & 781 & 59,7 & 26,4 \\
Pharmacy & 367 & 49,0 & 33,3 \\
\hline
\end{tabular}

Table 30. Faculty of Pharmacy. PP(int collab) and PP(industry) (within parentheses) by publication period. 3-year moving average.

\begin{tabular}{lcccccc}
\hline Department & $\mathbf{2 0 1 0 - 2 0 1 2}$ & $\mathbf{2 0 1 1 - 2 0 1 3}$ & $\mathbf{2 0 1 2 - 2 0 1 4}$ & $\mathbf{2 0 1 3 - 2 0 1 5}$ & $\mathbf{2 0 1 4 - 2 0 1 6}$ & $\mathbf{2 0 1 5 - 2 0 1 7}$ \\
\hline $\begin{array}{l}\text { Medicinal } \\
\text { Chemistry }\end{array}$ & $49,7(28,9)$ & $50,3(28,7)$ & $43,3(25,6)$ & $43,4(20,1)$ & $45,8(14,8)$ & $50,0(12,7)$ \\
$\begin{array}{l}\text { Pharmaceutical } \\
\text { Biosciences }\end{array}$ & & & & & & \\
Pharmacy & $60,4(30,2)$ & $59,7(28,2)$ & $62,8(27,0)$ & $59,5(26,9)$ & $61,4(24,9)$ & $58,8(23,3)$ \\
\hline
\end{tabular}

\section{Faculty of Medicine}

Table 31. Faculty of Medicine. Publications (P, full counts), PP(int collab) and PP(industry) for the whole publication period 2010-2017.

\begin{tabular}{lccc}
\hline Department & \multicolumn{3}{c}{ Indicator } \\
& P & PP(int collab) & PP(industry) \\
\hline Immunology, Genetics and Pathology & 2624 & 58,9 & 14,7 \\
Medical Biochemistry and Microbiology & 924 & 67,1 & 6,4 \\
Medical Cell Biology & 421 & 46,8 & 5,9 \\
Medical Sciences & 3892 & 54,9 & 15,4 \\
Neuroscience & 1853 & 47,3 & 4,3 \\
Public Health and Caring Sciences & 1639 & 46,4 & 10,7 \\
Surgical Sciences & 2398 & 48,2 & 10,2 \\
Women's and Children's Health & 1670 & 46,2 & 4,5 \\
\hline
\end{tabular}

Table 32. Faculty of Medicine. PP(int collab) and PP(industry) (within parentheses) by publication period. 3-year moving average.

\begin{tabular}{|c|c|c|c|c|c|c|}
\hline \multicolumn{7}{|l|}{ Department } \\
\hline \multicolumn{7}{|l|}{ Immunology, } \\
\hline \multicolumn{7}{|l|}{ Genetics and } \\
\hline Pathology & $57,1(16,6)$ & $56,5(15,3)$ & $57,2(13,7)$ & $57,6(13,0)$ & $60,3(13,0)$ & $61,5(13,4)$ \\
\hline \multicolumn{7}{|l|}{ Medical } \\
\hline \multicolumn{7}{|l|}{ Biochemistry and } \\
\hline \multicolumn{7}{|l|}{ Medical Cell } \\
\hline Biology & $52,1(9,7)$ & $46,1(7,1)$ & $45,3(7,3)$ & $40,2(4,8)$ & $44,8(3,4)$ & $45,6(2,8)$ \\
\hline Medical Sciences & $51,3(13,4)$ & $54,3(14,4)$ & $54,4(15,8)$ & $55,2(16,5)$ & $56,6(17,7)$ & $57,7(16,0)$ \\
\hline Neuroscience & $51,4(4,9)$ & $48,3(5,0)$ & $43,0(4,3)$ & $42,5(4,1)$ & $45,1(3,1)$ & $48,3(3,7)$ \\
\hline \multicolumn{7}{|l|}{ Public Health and } \\
\hline Caring Sciences & $45,2(12,9)$ & $44,1(9,3)$ & $46,7(9,8)$ & $46,8(9,8)$ & $48,2(9,3)$ & $46,8(9,3)$ \\
\hline Surgical Sciences & $48,7(11,6)$ & $46,8(13,4)$ & $44,4(11,8)$ & $45,6(10,2)$ & $48,4(8,8)$ & $51,1(8,5)$ \\
\hline \multicolumn{7}{|l|}{ Women's and } \\
\hline Children's Health & $40,7(4,1)$ & $42,3(3,8)$ & $43,7(4,4)$ & $46,9(5,7)$ & $48,4(5,8)$ & $50,7(4,9)$ \\
\hline
\end{tabular}




\subsubsection{Open access}

For both figures in this section, proportion $\mathrm{OA}$ is given as percentages. OA is defined as publications belonging to any class of OA (Gold, green or hybrid). All OA measures use full counts.

\section{Faculty of Pharmacy}

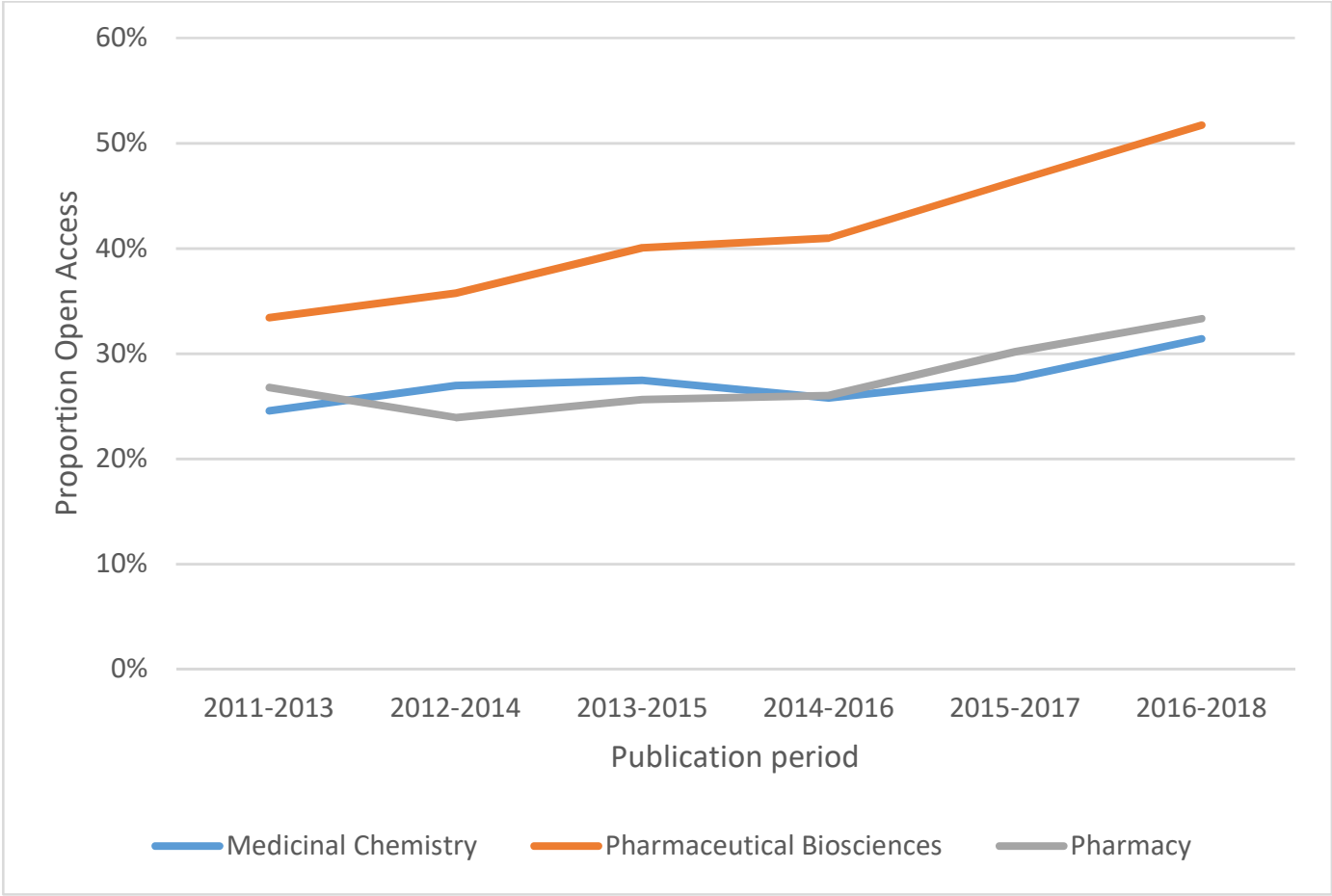

Figure 34. Faculty of Pharmacy. Total Open Access by publication period. 3-year moving average.

\section{Faculty of Medicine}

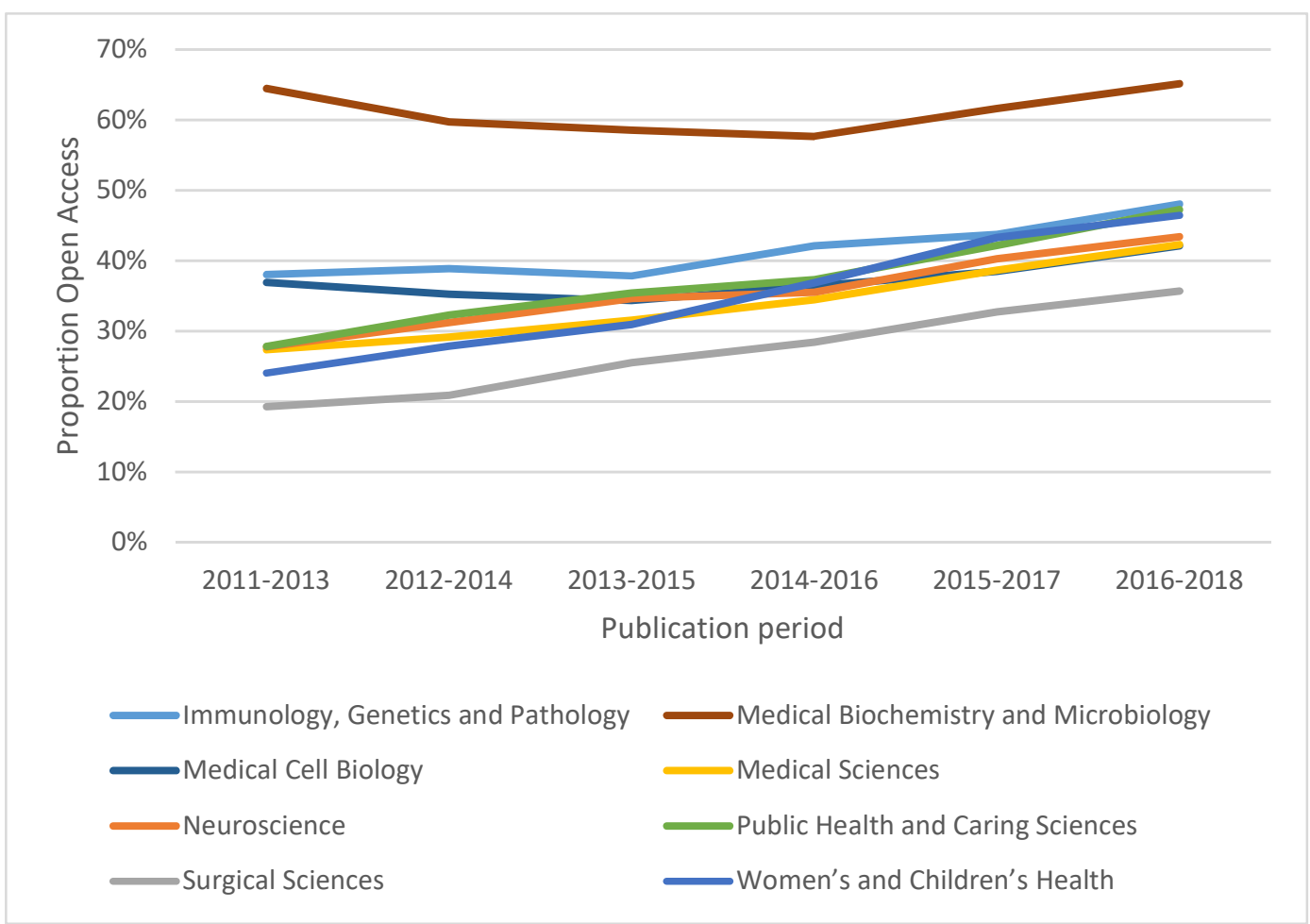

Figure 35. Faculty of Medicine. Total Open Access by publication period. 3-year moving average. 


\subsection{TekNat}

The number of TekNat departments included in ABM is 10. Table 33 reports publication volume, fractional counts and full counts (within parentheses) by department and publication type, and WoS coverage. WoS coverage concerns the number of WoS publications of the four types represented in the table relative to all publications of these types for the department.

Table 33. Faculty of Science and Technology. Publication volume by publication type, and WoS coverage (in \%). Publication period: 2010-2018. Data source: GLIS.

\begin{tabular}{|c|c|c|c|c|c|c|}
\hline \multirow{2}{*}{ Department } & \multicolumn{6}{|c|}{ Publication type } \\
\hline & Article & $\begin{array}{l}\text { Article in } \\
\text { anthology }\end{array}$ & Monograph & $\begin{array}{c}\text { Conference } \\
\text { paper }\end{array}$ & Total & $\begin{array}{l}\text { WoS } \\
\text { coverage }\end{array}$ \\
\hline $\begin{array}{l}\text { Cell and Molecular } \\
\text { Biology }\end{array}$ & $438,5(1128)$ & $18,1(25)$ & $1,2(2)$ & $4,9(15)$ & $\begin{array}{c}462,6 \\
(1170)\end{array}$ & 91,9 \\
\hline $\begin{array}{l}\text { Chemistry - BMC } \\
\text { Chemistry - }\end{array}$ & $272,3(730)$ & $7,2(14)$ & $1,0(1)$ & $2,2(6)$ & $282,6(751)$ & 91,7 \\
\hline Ångström & & & & & 825,6 & \\
\hline Laboratory & $784,3(1605)$ & $27,3(44)$ & $5,0(8)$ & $9,1(25)$ & $\begin{array}{l}(1682) \\
1036,0\end{array}$ & 90,9 \\
\hline $\begin{array}{l}\text { Genetics } \\
\text { Engineering }\end{array}$ & 888,6 (2074) & $19,8(41)$ & $1,8(3)$ & $0,4(1)$ & $\begin{array}{l}(2119) \\
2285,3\end{array}$ & 92,4 \\
\hline $\begin{array}{l}\text { Sciences } \\
\text { Information }\end{array}$ & $1567,4(2754)$ & $120,9(159)$ & $20,9(32)$ & $576,1(862)$ & $\begin{array}{l}(3807) \\
1525,8\end{array}$ & 60,9 \\
\hline Technology & $578,3(1131)$ & $73,2(102)$ & $5,5(9)$ & $868,8(1365)$ & (2607) & 56,3 \\
\hline $\begin{array}{l}\text { Mathematics } \\
\text { Organismal }\end{array}$ & $511,5(839)$ & $9,2(10)$ & $5,0(6)$ & $10,9(17)$ & $536,5(872)$ & 85,2 \\
\hline $\begin{array}{l}\text { Biology } \\
\text { Physics and }\end{array}$ & $370,8(880)$ & $38,7(47)$ & $2,2(3)$ & $29,5(42)$ & $\begin{array}{c}441,1(972) \\
2720,0\end{array}$ & 69,6 \\
\hline Astronomy & $2451,0(5744)$ & $46,3(59)$ & $8,2(10)$ & $214,5(412)$ & $(6225)$ & 85,5 \\
\hline
\end{tabular}


3.4.1 Publishing volume and publishing level-The Norwegian model

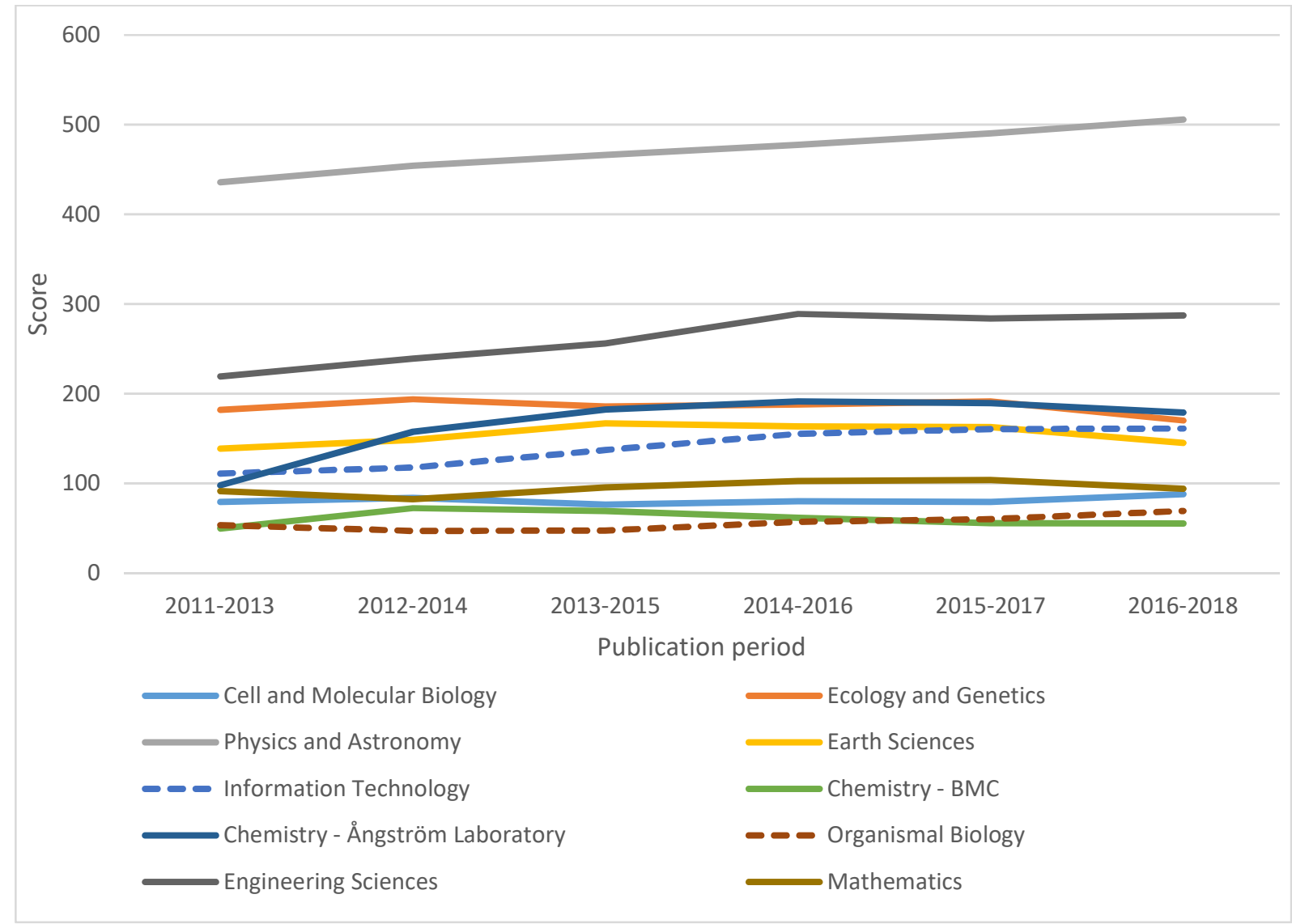

Figure 36. Faculty of Science and Technology. Norwegian score by publication period. 3-year moving average. 
Table 34. Faculty of Science and Technology. Proportion (in \%) publication fractions at level 2 relative to the sum of publication fractions across levels 1 and 2 (given within parentheses) by publication period. 3-year moving average.

\begin{tabular}{|c|c|c|c|c|c|c|}
\hline Department & 2011-2013 & 2012-2014 & 2013-2015 & 2014-2016 & 2015-2017 & 2016-2018 \\
\hline $\begin{array}{l}\text { Cell and } \\
\text { Molecular }\end{array}$ & & & & & & \\
\hline $\begin{array}{l}\text { Biology } \\
\text { Chemistry- }\end{array}$ & $44,2(125,9)$ & $44,1(132,1)$ & $35,0(133,2)$ & $32,7(145,0)$ & $30,9(148,6)$ & $30,4(165,2)$ \\
\hline $\begin{array}{l}\text { BMC } \\
\text { Chemistry - } \\
\text { Ångström }\end{array}$ & $36,1(84,9)$ & $35,9(124,8)$ & $35,5(122,0)$ & $33,5(111,3)$ & $31,3(103,3)$ & $29,9(104,6)$ \\
\hline $\begin{array}{l}\text { Laboratory } \\
\text { Earth }\end{array}$ & $31,1(181,3)$ & $31,9(288,8)$ & $33,3(327,3)$ & $31,7(350,2)$ & $29,5(352,1)$ & $25,4(350,9)$ \\
\hline $\begin{array}{l}\text { Sciences } \\
\text { Ecology and }\end{array}$ & $28,0(269,2)$ & $29,3(283,5)$ & $30,4(313,7)$ & $31,2(302,5)$ & $29,3(309,5)$ & $27,4(283,2)$ \\
\hline $\begin{array}{l}\text { Genetics } \\
\text { Engineering }\end{array}$ & $38,1(309,8)$ & $39,6(326,0)$ & $41,3(305,8)$ & $45,1(299,5)$ & $47,5(297,0)$ & $44,7(272,2)$ \\
\hline $\begin{array}{l}\text { Sciences } \\
\text { Information }\end{array}$ & $23,5(450,7)$ & $24,0(489,0)$ & $22,8(530,6)$ & $24,9(583,1)$ & $23,8(585,1)$ & $23,8(587,6)$ \\
\hline Technology & $18,0(255,1)$ & $18,6(268,5)$ & $17,4(321,1)$ & $19,1(355,2)$ & $16,5(383,6)$ & $16,9(379,8)$ \\
\hline $\begin{array}{l}\text { Mathematics } \\
\text { Organismal }\end{array}$ & $34,3(157,9)$ & $28,4(154,1)$ & $29,6(180,7)$ & $34,3(183,4)$ & $34,2(185,1)$ & $35,4(165,8)$ \\
\hline $\begin{array}{l}\text { Biology } \\
\text { Physics and }\end{array}$ & $22,9(112,7)$ & $22,8(99,6)$ & $23,4(97,7)$ & $25,0(115,3)$ & $24,0(123,0)$ & $30,1(130,7)$ \\
\hline Astronomy & $36,8(754,9)$ & $38,7(769,4)$ & $40,1(779,1)$ & $39,2(807,4)$ & $37,1(849,0)$ & $36,3(880,5)$ \\
\hline
\end{tabular}

\subsubsection{Field normalized citation impact}

Table 35. Faculty of Science and Technology. Publication fractions (P; full counts within parentheses), MNCS, PP(top 10\%), MNJS and MJPPtop10\% for the whole publication period 2010-2017.

\begin{tabular}{lccccc}
\hline Department & P & MNCS & $\begin{array}{r}\text { Indicator } \\
\text { PP(top 10\%) }\end{array}$ & MNJS & MJPPtop10\% \\
\hline Cell and Molecular Biology & $330,6(907)$ & 1,50 & 16,7 & 1,52 & 16,6 \\
Chemistry - BMC & $309,9(818)$ & 0,86 & 8,7 & 1,03 & 10,9 \\
Chemistry - Ångström Laboratory & $757,0(1604)$ & 1,09 & 11,9 & 1,12 & 11,4 \\
Earth Sciences & $553,6(1567)$ & 0,93 & 7,7 & 1,01 & 10,1 \\
Ecology and Genetics & $697,1(1750)$ & 1,57 & 17,7 & 1,52 & 17,1 \\
Engineering Sciences & $1040,9(1931)$ & 0,91 & 7,4 & 1,05 & 10,3 \\
Information Technology & $377,6(794)$ & 0,99 & 9,7 & 0,98 & 9,7 \\
Mathematics & $364,1(647)$ & 1,06 & 10,1 & 1,08 & 11,0 \\
Organismal Biology & $232,6(637)$ & 1,51 & 15,7 & 1,48 & 14,1 \\
Physics and Astronomy & $1268,6(4882)$ & 1,00 & 10,4 & 1,04 & 10,2 \\
\hline
\end{tabular}




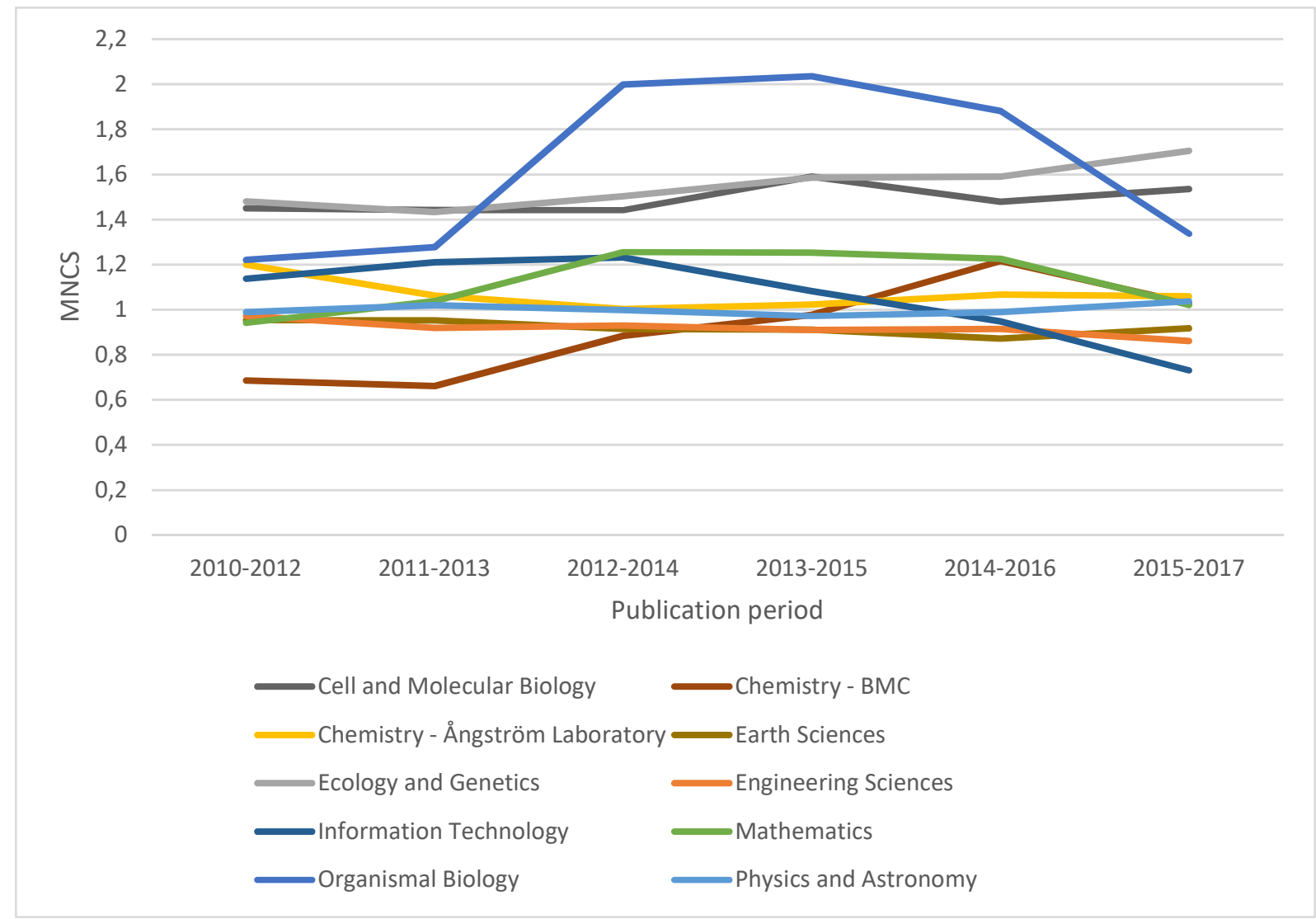

Figure 37. Faculty of Science and Technology. MNCS by publication period. 3-year moving average.

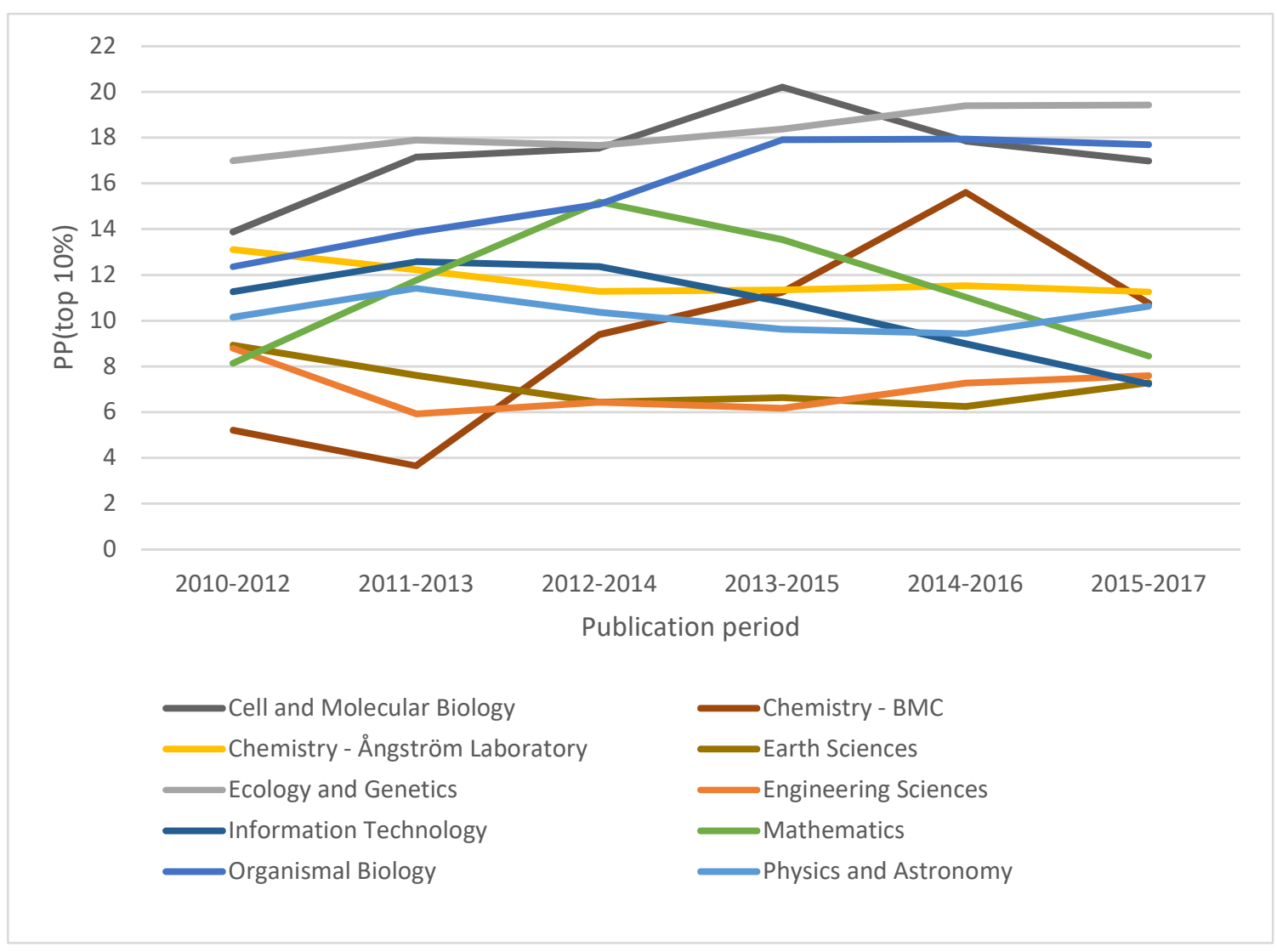

Figure 38. Faculty of Science and Technology. PP(top 10\%) by publication period. 3-year moving average. 


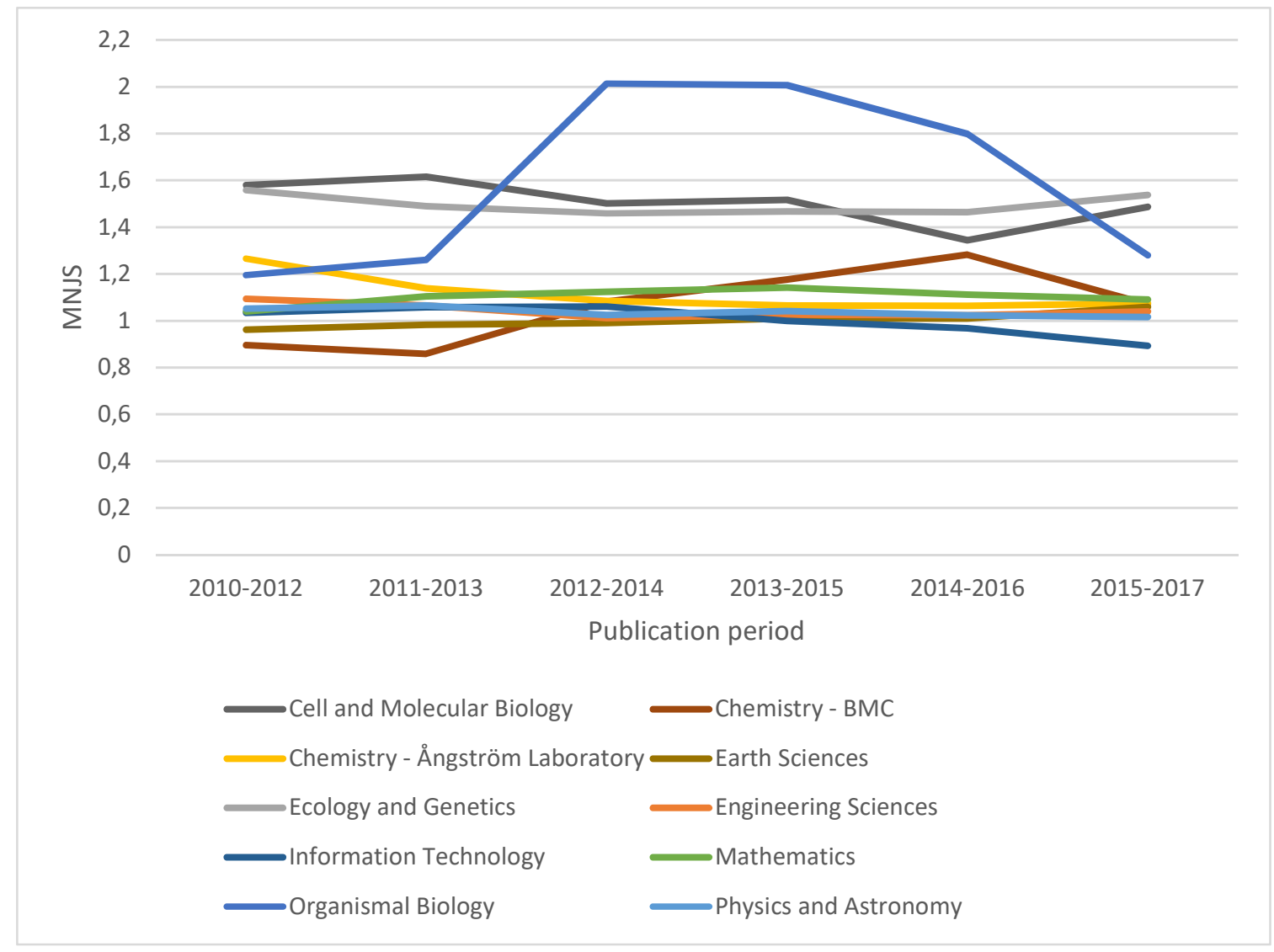

Figure 39. Faculty of Science and Technology. MNJS by publication period. 3-year moving average.

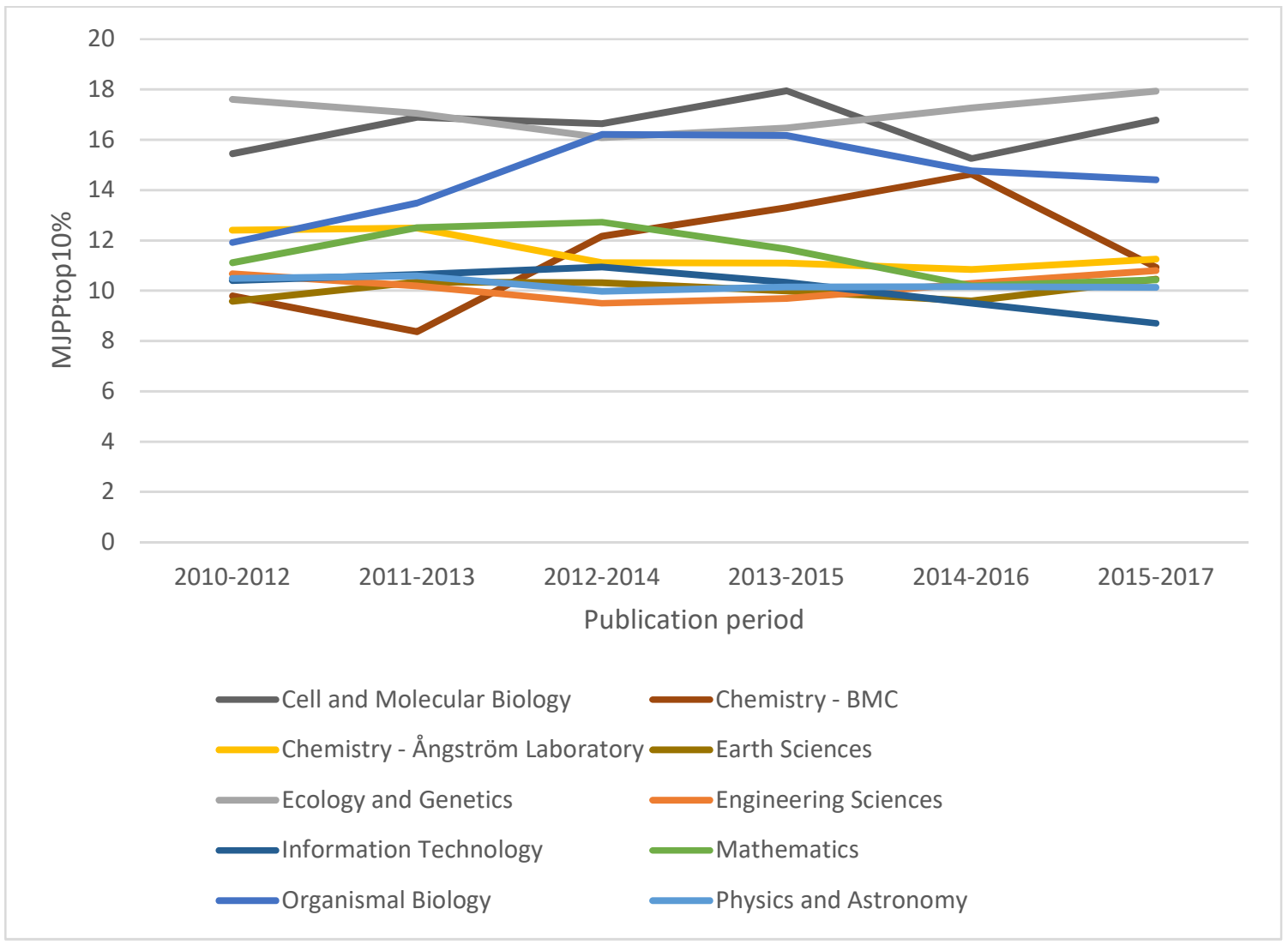

Figure 40. Faculty of Science and Technology. MJPPtop10\% by publication period. 3 -year moving average. 


\subsubsection{Collaboration}

For all tables in this section, $\mathrm{PP}($ int collab) and PP(industry) are given as percentages.

Table 36. Faculty of Science and Technology. Publications (P, full counts), PP(int collab) and PP(industry) for the whole publication period 2010-2017.

\begin{tabular}{lccc}
\hline Department & \multicolumn{3}{c}{ Indicator } \\
& P & PP(int collab) & PP(industry) \\
\hline Cell and Molecular Biology & 907 & 65,8 & 8,0 \\
Chemistry - BMC & 1604 & 60,0 & 12,5 \\
Chemistry - Angström Laboratory & 818 & 54,2 & 4,9 \\
Earth Sciences & 1567 & 81,2 & 5,4 \\
Ecology and Genetics & 1750 & 74,9 & 3,0 \\
Engineering Sciences & 1931 & 50,0 & 11,6 \\
Information Technology & 794 & 57,9 & 6,0 \\
Mathematics & 647 & 61,8 & 1,5 \\
Organismal Biology & 637 & 72,2 & 2,5 \\
Physics and Astronomy & 4882 & 86,7 & 3,8 \\
\hline
\end{tabular}

Table 37. Faculty of Science and Technology. PP(int collab) and PP(industry) (within parentheses) by publication period. 3-year moving average.

\begin{tabular}{lcccccc}
\hline Department & $\mathbf{2 0 1 0 - 2 0 1 2}$ & $\mathbf{2 0 1 1 - 2 0 1 3}$ & $\mathbf{2 0 1 2 - 2 0 1 4}$ & $\mathbf{2 0 1 3 - 2 0 1 5}$ & $\mathbf{2 0 1 4 - 2 0 1 6}$ & $\mathbf{2 0 1 5 - 2 0 1 7}$ \\
\hline Cell and Molecular & & & & & & \\
Biology & $62,8(12,3)$ & $62,6(10,9)$ & $63,9(8,8)$ & $66,7(6,0)$ & $67,6(5,3)$ & $68,1(4,6)$ \\
Chemistry - BMC & $55,8(17,7)$ & $56,6(12,9)$ & $51,5(10,4)$ & $48,8(7,2)$ & $52,4(10,0)$ & $56,3(9,9)$ \\
$\begin{array}{l}\text { Chemistry - } \\
\text { Angström }\end{array}$ & & & & & & \\
Laboratory & $59,2(5,5)$ & $60,5(5,0)$ & $59,6(5,7)$ & $58,8(4,2)$ & $60,2(4,4)$ & $60,9(4,6)$ \\
Earth Sciences & $83,2(6,0)$ & $83,1(5,1)$ & $80,9(5,3)$ & $79,4(4,9)$ & $80,3(5,5)$ & $81,0(5,1)$ \\
Ecology and & $71,5(2,9)$ & $72,4(1,9)$ & $73,4(2,5)$ & $75,3(2,8)$ & $77,6(3,9)$ & $78,9(3,3)$ \\
Genetics & & & & & & \\
Engineering & $45,8(14,3)$ & $45,2(12,6)$ & $46,0(11,1)$ & $48,4(10,8)$ & $51,1(10,9)$ & $54,0(10,5)$ \\
Sciences & $51,3(6,6)$ & $52,5(5,5)$ & $56,9(3,8)$ & $61,5(4,8)$ & $60,7(5,5)$ & $59,8(7,5)$ \\
Information & $57,8(1,5)$ & $63,1(0,9)$ & $65,1(0,9)$ & $62,7(1,5)$ & $60,1(2,2)$ & $63,9(2,1)$ \\
Technology & & & & & \\
Mathematics & $65,4(3,2)$ & $67,0(1,6)$ & $71,9(0,5)$ & $69,2(0,9)$ & $73,5(1,5)$ & $78,1(2,8)$ \\
Organismal & & & & & \\
Biology & $85,5(3,3)$ & $86,5(3,1)$ & $87,3(3,5)$ & $88,0(4,1)$ & $87,9(4,6)$ & $87,1(4,3)$ \\
$\begin{array}{l}\text { Physics and } \\
\text { Astronomy }\end{array}$ & & & & & \\
\hline
\end{tabular}

\subsubsection{Open access}

For Figure 41, proportion OA is given as percentages. OA is defined as publications belonging to any class of OA (Gold, green or hybrid). All OA measures use full counts. 


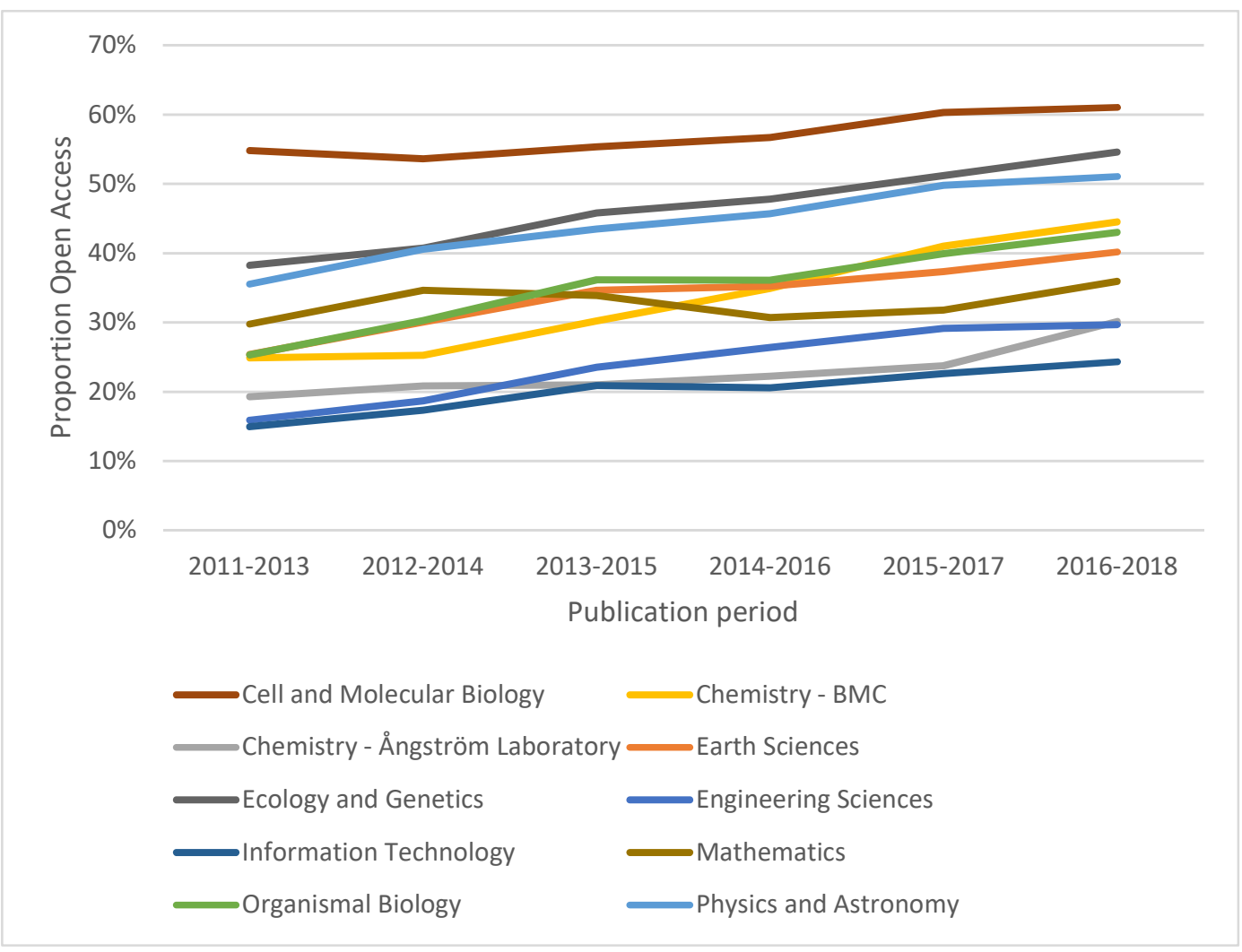

Figure 41. Faculty of Science and Technology. Total Open Access by publication period. 3-year moving average.

\section{Brief discussion of the results}

\section{HumSam}

One interesting trend is the increase in WoS coverage noticed for some departments in the Faculty of Arts. This result is further examined in Appendix 1.

Regarding the Norwegian model, the increase in proportion of publication fractions at level 2 for some departments in the Faculty of Arts is noteworthy (Table 7). Archeology and Ancient History, History and Philosophy all have an increase in the interval 18-20 percentage units from the publication period 2011-2013 to the period 2016-2018. This is also the case for English in the Faculty of Languages (Table 8). In addition, Faculty of Education and Faculty of Theology show an increase in the proportion of publication fractions at level 2 (of about 17 and 15 percentage units, respectively) (Table 9). In the Faculty of Social Sciences, the increase is most noteworthy for Economic History (25 percentage units), Economics (18 percentage units), Peace and Conflict Studies (16 percentage units) and Social and Economic Geography (14 percentage units) (Table 10).

When it comes to citation indicators, Peace and Conflict Studies is worth mentioning, since the department has very high figures for all four indicators with respect to the whole publication period (Table 14). Moreover, the proportion of author fractions at level 2 is also high for this department, especially for the period 2016-2018 (about 58\%; Table 10). Thus, there is, for this department, an agreement between the citation impact performance and the Norwegian model performance.

The extreme OA trend for Game Design under the Faculty of Arts is mostly explained by a two-year period with approximately 50 book chapters made freely available. The faculty overall has an increasing trend in OA, apart from Game Design (Figure 20). Further, the Faculty of Education has a remarkably increasing development (Figure 22). 


\section{MedFarm}

For the Faculty of Pharmacy, the Pharmacy department has very high values on the MNCS and PP(top $10 \%$ ) indicators, especially in the last two publication periods (Figures 26 and 27). With regard to the Norwegian model, Pharmacy has a quite high proportion of author fractions at level 2, and this result is most pronounced in the period 2016-2018 (Table 25).

Regarding the Faculty of Medicine, the highest values on the citation indicators, with respect to the whole publication period, are observed for the two departments Medical Biochemistry and Microbiology and Immunology, Genetics and Pathology (Table 28). Regardless of citation indicator, the latter department has an upward trend across the six considered periods, while the former one has quite stable values across the six periods (Figures 30-33). Medical Biochemistry and Microbiology has an unparalleled high OA level compared to all other institutions at UU throughout the entire time period (Figure 35).

\section{TekNat}

For the Faculty of Science and Technology, the three biology departments, Cell and Molecular Biology, Ecology and Genetics and Organismal Biology, all have high values on the citation indicators with respect to the whole period (Table 35). With regard to the Norwegian model, Ecology and Genetics shows high figures with regard to proportion of author fractions at level 2 (Table 34).

Noteworthy is also the high values on the international collaboration indicator, PP(int collab), for the two departments Earth Sciences and Physics and Astronomy (Tables 36 and 37).

The overall trend for OA is increasing for the entire faculty. Noteworthy is the department of Cell and Molecular Biology, which has the second highest proportion of OA at UU during the entire time series (Figure 41).

\section{Concluding remarks}

In this report, we have we have described the data and methods used in UU ABM 2019, and we have reported results on publication volume, publishing level, citation impact, collaboration (in terms of copublishing) and open access. For the measurement of citation impact, four advanced bibliometric indicators were used.

We believe that the results of ABM are of interest, not only for the university management, but also for the heads of the departments of UU. However, bibliometric statistics should be interpreted with great caution when underlying publication sets are small. For some of the departments covered by ABM, this is the case.

In our view, and as is pointed out in the Leiden Manifesto for research metrics (Hicks et al., 2015), bibliometric statistics should support peer review assessments, not replace such assessments. The use of bibliometric statistics can indeed strengthen peer review, since such statistics can be used to question bias tendencies in peer review. As is also pointed out by Leiden Manifesto for research metrics, variation by field in publication and citation practices should be controlled for in bibliometric research evaluation. In UU ABM 2019, and with respect to citation impact, this principle is taken into account.

\section{References}

Hicks, D., Wouters, P., Waltman, L., de Rijcke, S., and Rafols, I. (2015). The Leiden Manifesto for research metrics. Nature, 520(7548), 429-431.

Malmberg, A., Kettis, Å., \& Maandi, C. (Eds.). (2017). Quality and Renewal 2017 (Kvalitet och förnyelse 2017) : Research Environment Evaluation at Uppsala University. Uppsala. 
Prop. 2016/17:50. Kunskap i samverkan - för samhällets utmaningar och stärkt konkurrenskraft. https://www.regeringen.se/4adad0/contentassets/72faaf7629a845af9b30fdelef6b5067/kunskap-isamverkan--for-samhallets-utmaningar-och-starkt-konkurrenskraft-prop.-20161750.pdf

Schneider, J. W. (2009). An outline of the bibliometric indicator used for performance-based funding of research institutions in Norway. European Political Science, 8(3), 364-378.

van Eck, N. J., Waltman, L., van Raan, A. F. J., Klautz, R. J. M., \& Peul, W. C. (2013). Citation analysis may severely underestimate the impact of clinical researchas compared to basic research. PLOS $O N E, 8(4)$, e 62395.

Waltman, L., \& van Eck, N. J. (2012). A new methodology for constructing a publication-level classification system of science. Journal of the American Society for Information Science and Technology, 63(12), 2378-2392.

Waltman, L., \& Schreiber, M. (2013). On the calculation of percentile-based bibliometric indicators. Journal of the American Society for Information Science and Technology, 64(2), 372-379.

\section{Appendix 1 Faculty of Arts: WoS coverage for a sample of departments}

An interesting result in ABM is the substantial increase in WoS coverage for some of the departments within the humanities. In Figure 42, the WoS coverage for the publication period 2010-2018 is presented for those departments of the Faculty of Arts that have a substantial increase in coverage. The vertical axis in the diagram of the figure represents index numbers, where the base period is set to the period 2010-2012. Note that some departments have a rather low output (for instance Art History and Cultural Anthropology and Ethnology), so the coverage values should be interpreted with caution. A few departments, like $A B M$ and Philosophy, have a large proportion of their articles represented in WoS but show no, or a small, increase across the periods. In view of this, such departments are not represented in the figure.

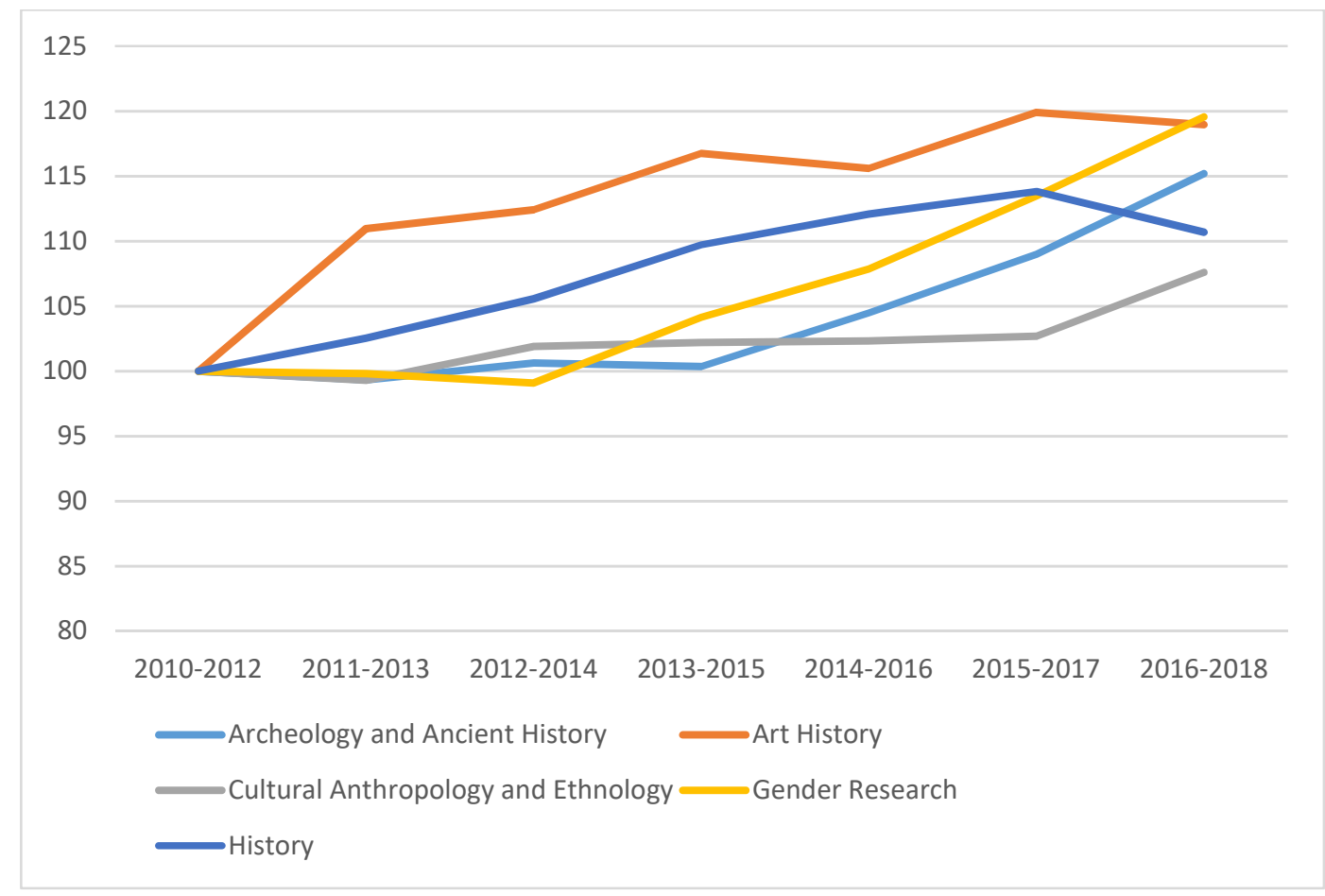

Figure 42. Sample humanities departments. WoS coverage by publication period. Index numbers, base period $=$ 2010-2012. 3-year moving average. Data source: GLIS. 
All five departments represented in the figure show a quite remarkable increase in publishing articles in journals covered by WoS (and the same trend is also notable for a few departments belonging to other faculties in the scientific domain HumSam). For example, three of the five departments have increased their coverage with $15 \%$ or more relative to the base period. In later years, we have observed a decrease in the publishing of books and articles in anthologies. This might indicate a shift in the publishing patterns in at least some parts of HumSam.

\section{Appendix 2 Formal definitions of citation indicators used in UU ABM}

In this appendix, we describe the calculation of the four field normalized citation impact indicators used in UU ABM.

The following indicators are defined in this appendix:

- Mean normalized number of citations per publication (MNCS).

- Proportion of frequently cited publications (top 10\%) (PP(top 10\%)).

- Mean normalized journal impact score per publication (MNJS).

- Mean journal proportion of top $10 \%$ publications (MJPP(top 10\%)).

MNCS and PP(top 10\%) are publication-level indicators, whereas MNJS and MJPP(top 10\%) are journal-level indicators.

Regardless of indicator, a subject classification of publications is used for field normalization purposes. The classification is generated by application of an advanced clustering methodology, in which a publication-publication relatedness measure based on direct citations between publications is utilized (Waltman \& van Eck, 2012). The classification, which contains about 4,000 classes, is such that each publication belongs to exactly one class. ${ }^{12}$ The classification has been created by CWTS, Leiden University, Netherlands.

This appendix treats the case, in which fractional counts are used in the calculations of indicator values. In case whole counts should be used in the calculations, $a_{i}$ in Eq. (1) below is set to 1.

Let A be a unit of analysis, and $n$ the number of publications for A. Let $r_{i}$ be the number of authors of the $i$ th publication for A. Let $a_{i}$ be the author fraction A has of the $i$ th publication. We consider two cases.

(a) A is an organization, for instance a department. $a_{i}$ is then defined as

$$
a_{i}=\sum_{j=1}^{m_{i}} \frac{1}{r_{i} s_{j}}
$$

where $m_{i}$ is the number of authors affiliated to A regarding the $i$ th publication, and $s_{j}$ the number of affiliations of the $j$ th of these A authors. Note that the right-hand side in Eq. (1) is equal to $m_{i} / r_{i}$ when each A author has exactly one affiliation.

(b) $\mathrm{A}$ is an individual author. $a_{i}$ is in this case defined as $1 / r_{i}$.

We define MNCS for A, MNCS(A), as

\footnotetext{
${ }^{12} \mathrm{Cf}$. footnote 4 in this report.
} 


$$
\begin{gathered}
\operatorname{MNCS}(\mathrm{A})=\frac{\sum_{i=1}^{n} a_{i} \mathrm{NCS}_{i}}{\sum_{i=1}^{n} a_{i}} \\
\mathrm{NCS}_{i}=c_{i} / \mu_{s c_{-} i} \\
\mu_{s c_{-} i}=\frac{1}{k_{i}} \sum_{j=1}^{k_{i}} c_{j}
\end{gathered}
$$

where $\mathrm{NCS}_{i}$ is the normalized citation score for the $i$ th publication of $\mathrm{A}, c_{i}$ is the citation score of the $i$ th publication, $s c \_i$ the subject class to which the $i$ th publication has been (algorithmically) assigned, $k_{i}$ the number of publications in $s c$ _ $i$ with the same publication year as the $i$ th publication, and $c_{j}$ the citation score of the $j$ th publication among these latter publications. $\mu_{s c_{i} i}$ is the field reference value that $c_{i}$ is normalized against. The normalization gives rise to a normalized citation score for the $i$ th publication. Clearly, MNCS(A) is a weighted mean across the publications of A, a fact that also holds for the other three citation indicators treated in this document (Sections 2.2-2.4).

We define $\mathrm{PP}($ top $10 \%)$ for $\mathrm{A}, \mathrm{PP}($ top $10 \%)(\mathrm{A})$, as

$$
\begin{gathered}
\operatorname{PP}(\operatorname{top} 10 \%)(\mathrm{A})=\frac{\sum_{i=1}^{n} a_{i} b_{i}}{\sum_{i=1}^{n} a_{i}} \\
b_{i}=\frac{\max \left(y_{s c_{-} i}^{c_{i}+1}-\max \left(0.9, y_{s c_{-} i}^{c_{i}}\right), 0\right)}{y_{s c_{-} i}^{c_{c_{i}+1}}-y_{s c_{-} i}^{c_{c_{i}}}}
\end{gathered}
$$

where $y_{s c_{-} i}^{c_{i}}\left(y_{s c_{-} i}^{c_{i}+1}\right)$ is the proportion publications-with respect to the citation distribution, which concerns the publications in $s c_{-} i$ with the same publication year as the $i$ th publication of A-with less than $c_{i}\left(c_{i}+1\right)$ citations. $\max \left(y_{s c_{-} i}^{c_{i}+1}-\max \left(0.9, y_{s c_{-} i}^{c_{i}}\right), 0\right) / y_{s c_{-} i}^{c_{i}+1}-y_{s c_{-} i}^{c_{i}}$ is the fraction of the $i$ th publication with which the publication is assigned to the $10 \%$ most frequently cited publications (Waltman \& Schreiber, 2013).

We define MNJS for A, MNJS(A), as

$$
\begin{gathered}
\operatorname{MNJS}(\mathrm{A})=\frac{\sum_{i=1}^{n} a_{i} \mathrm{NJS}_{i}}{\sum_{i=1}^{n} a_{i}} \\
\mathrm{NJS}_{i}=\frac{\sum_{j=1}^{p_{i}} \mathrm{NCS}_{j}}{p_{i}} \\
\mathrm{NCS}_{j}=c_{j} / \mu_{s c j} \\
\mu_{s c j}=\frac{1}{k_{j}} \sum_{l=1}^{k_{j}} c_{l}
\end{gathered}
$$

where $\mathrm{NJS}_{i}$ is the normalized journal score of the journal, say $J_{i}$, of the $i$ th publication, $p_{i}$ the number of publications in $J_{i}, \operatorname{NCS}_{j}\left(c_{j}\right)$ the normalized citation score (citation score) of the $j$ th publication in $J_{i}$, say $P_{j}, s c_{j} j$ the subject class to which $P_{j}$ has been assigned, $k_{j}$ the number of publications in $s c j j$ with the 
same publication year as $P_{j}$, and $c_{l}$ the citation rate of the $l$ th of these latter publications. $\mu_{s c_{j} j}$ is the field reference value that the citation score of $P_{j}, c_{j}$, is normalized against, and the normalization gives rise to a field normalized citation score for $P_{j}$ (cf. the definition of MNCS above).

We define the MJPPtop10\% for A, MJPP(top 10\%)(A), as

$$
\begin{gathered}
\operatorname{MJPP}\left(\text { top 10\%)(A) }=\frac{\sum_{i=1}^{n} a_{i} \operatorname{JPP}(\text { top } 10 \%)_{i}}{\sum_{i=1}^{n} a_{i}}\right. \\
\operatorname{JPP}(\operatorname{top} 10 \%)_{i}=\frac{\sum_{j=1}^{q_{i}} b_{j}}{k_{i}} \\
b_{j}=\frac{\max \left(y_{s c_{-} i}^{c_{j}+1}-\max \left(0.9, y_{s c_{-} i}^{c_{j}}\right), 0\right)}{y_{s c_{-} i}^{c_{j}+1}-y_{s c_{-} i}^{c_{j}}}
\end{gathered}
$$

where $k_{i}$ is as in Eq. (2), $q_{i}$ the number of publications in $s c i$ with (a) the same publication year as the $i$ th publication of $\mathrm{A}$, and (b) belonging to the journal of the $i$ th publication of $\mathrm{A}$. $\max \left(y_{s \__{-} i}^{c_{j}+1}-\max \left(0.9, y_{s c_{-} i}^{c_{c_{j}}}\right), 0\right) / y_{s c_{-} i}^{c_{j}+1}-y_{s c_{-} i}^{c_{j}}$ is the fraction of the $j$ th publication, in $s c_{-} i$ and satisfying the conditions (a) and (b), with which the publication is assigned to the $10 \%$ most frequently cited publications with respect to the citation distribution determined by $s c$ i and the publication year of the $i$ th publication of A.

$\operatorname{JPP}($ top $10 \%) i$ is then the extent, on average, to which the publications that belong to $s c$, with the same publication year as the $i$ th publication of $\mathrm{A}$ and belonging to the same journal as the $i$ th publication of A, belong to the $10 \%$ most frequently cited publications with respect to the citation distribution determined by $s c_{-} i$ and the publication year of the $i$ th publication. 